This is a post-peer-review, pre-copyedit version of an article published in Pharmaceutical Research. The final authenticated version is available online at: https://doi.org/10.1007/s11095-011-0568-5 


\title{
Click Chemistry for Drug Delivery Nanosystems
}

Enrique Lallana, ${ }^{1}$ Ana Sousa-Herves, ${ }^{1}$ Francisco Fernandez-Trillo, ${ }^{1}$ Ricardo Riguera, ${ }^{1}$ and

\author{
Eduardo Fernandez-Megia ${ }^{1,2}$ \\ ${ }^{1}$ Department of Organic Chemistry and Center for Research in Biological Chemistry and \\ Molecular Materials (CIQUS), University of Santiago de Compostela, Jenaro de la Fuente s/n, \\ 15782 Santiago de Compostela, Spain \\ ${ }^{2}$ To whom correspondence should be addressed (e-mail:ef.megia@usc.es)
}

Running Title: Click Chemistry for Drug Delivery Nanosystems

Click Chemistry for Drug Delivery Nanosystems 


\section{Abbreviations}

AAC (azide-alkyne cycloaddition), AIBN (azobisisobutyronitrile), AgNP (silver nanoparticle), Alk (alkyne), ATRP (atom transfer radical polymerization), AuNP (gold nanoparticle), Az (azide), BNP (bionanoparticle), BPDS (bathophenanthroline disulphonated disodium salt), CD (cyclodextrin), CMC (critical micelle concentration), CNT (carbon nanotube), CPMV (cowpea mosaic virus), CTA (chain transfer agent), CuAAC [Cu(I)-catalyzed azide-alkyne cycloaddition], DBU (1,8-diazabicyclo[5.4.0]undec-7-ene), DDS (drug delivery system), DIPEA ( $N, N$ diisopropylethylamine), DOTA (1,4,7,10-tetraazacyclododecane-1,4,7,10-tetraacetic acid), DOX (doxorubicin), EGF (epidermal growth factor), EGFP (enhanced green fluorescent protein), EPL (expressed protein ligation), EPR (enhanced permeability and retention), FA (folic acid), FRET (fluorescence resonance energy transfer), FPLC (fast protein liquid chromatography), FR (folate receptor), GFP (green fluorescent protein), HABA [2-(4-hydroxyphenylazo)benzoic acid], HEMA (2-hydroxyethyl methacrylate), LA (lactic acid), LbL (layer-by-layer), MAA (methacrylic acid), MBP (maltose binding protein), MNP (magnetic nanoparticle), MRI (magnetic resonance imaging), MWCNT (multi-walled carbon nanotube), NHS ( $N$ hydroxysuccinimide), NP (nanoparticle), PACA [poly(alkyl cyanocrylate)], PAA [poly(acrylic acid)], PBD [poly(butadiene)], PCL [poly(ع-caprolactone)], PCN (polymer-caged nanobin), PDMA $[\operatorname{poly}(N, N$-dimethylacrylamide)], PEG [poly(ethylene glycol)], PEI [poly(ethylene imine)], PEO [poly(ethylene oxide)], PET (position emission tomography), PGA (poly-Lglutamic acid), PIC (polyion complex), PLL (poly-L-lysine), PMA [poly(methacrylate)], PMDETA ( $N, N, N^{\prime}, N^{\prime}, N^{\prime \prime}$-pentamethyldiethylenetriamine), PMPC [poly(2-methyl-2-carboxylpropylene carbonate)], PNIPAM [poly ( $N$-isopropylacrylamide)], PrMA (propyl methacrylate), PS [poly(styrene)], PTQY (photoluminescence quantum yield), PtNP (platinum nanoparticle),

\section{Click Chemistry for Drug Delivery Nanosystems}


PTMCC [poly(2-methyl-2-carboxytrimethylene carbonate)], PVP [poly(vinyl pyrrolidone)], QD (quantum dot), RAFT (reversible addition-fragmentation chain transfer), RGD (Arg-Gly-Asp), ROS (reactive oxygen species), SiNP (silica nanoparticle), SPAAC (strain-promoted azidealkyne cycloaddition), SPION (superparamagnetic iron oxide nanoparticle), STEM (scanning transmission electron microscopy), SWCNT (single-walled carbon nanotube), TBTA [tris(benzyltriazolylmethyl)amine], TCEP [tris(carboxyethyl)phosphine], TEC (thiol-ene coupling), THPTA [tris(hydroxypropyltriazolylmethyl)amine], TMS (trimethylsilyl), VNP (viral nanoparticle). 


\begin{abstract}
The purpose of this Expert Review is to discuss the impact of click chemistry in nanosized drug delivery systems. Since the introduction of the click concept by Sharpless and coworkers in 2001, numerous examples of click reactions have been reported for the preparation and functionalization of polymeric micelles and nanoparticles, liposomes and polymersomes, capsules, microspheres, metal and silica nanoparticles, carbon nanotubes and fullerenes, or bionanoparticles. Among these click processes, the $\mathrm{Cu}(\mathrm{I})$-catalyzed azide-alkyne cycloaddition (CuAAC) has attracted most attention based on its high orthogonality, reliability, and experimental simplicity for non-specialists. In addition, a renewed interest on the use of efficient classical transformations has been also observed (e.g., thiol-ene coupling, Michael addition, Diels-Alder). In this Expert Review special emphasis is also devoted to critically discuss the click concept, as well as practical aspects on the application of CuAAC to ensure an efficient and harmless bioconjugation.
\end{abstract}

Key Words: click chemistry; CuAAC; drug delivery; nanostructure; bioconjugation 
"The reaction must be modular, wide in scope, give very high yields, generate only inoffensive byproducts that can be removed by nonchromatographic methods, and be stereospecific (but not necessarily enantioselective). The required process characteristics include simple reaction conditions (ideally, the process should be insensitive to oxygen and water), readily available starting materials and reagents, the use of no solvent or a solvent that is benign (such as water) or easily removed, and simple product isolation. Purification - if required - must be by nonchromatographic methods, such as crystallization or distillation, and the product must be stable under physiological conditions... Click processes proceed rapidly to completion and also tend to be highly selective for a single product: we think of these reactions as being "spring-loaded" for a single trajectory". H. C. Kolb, M. G. Finn and K. B. Sharpless. Angew. Chem., Int. Ed. 40: 2004-2021 (2001).

\section{INTRODUCTION}

Over the past few decades, various generations of drug delivery systems (DDS) have been developed for the controlled administration of drugs into the body (1). Among them, nanosized carriers are particularly attractive since they provide protection to the cargo, while enhancing cellular uptake and the selective delivery to specific cells. Since their appearance in the 1970s, a number of nanosized DDS have been described as carriers for the delivery and controlled release of drugs, including drug-polymer conjugates and nanoparticulate carriers, where the drug is either physically entrapped or covalently attached to the delivery system (2).

The widespread application of DDS in nanomedicine has been prompted by several remarkable discoveries and breakthroughs, the most relevant being (1): (i) The aim of minimizing immunogenicity and preventing the early clearance of proteins, drugs, and DDS by

\section{Click Chemistry for Drug Delivery Nanosystems}


conjugation to hydrophilic biocompatible polymers, such as poly(ethylene glycol) (PEG) (3); (ii) The development of active targeting, that is the selective delivery of drugs to target tissues and cells facilitated by the privileged interaction of ligands on the periphery of drug carriers with cell surface receptors (4); (iii) The discovery of the enhanced permeability and retention (EPR) effect, also known as passive targeting to explain the selective accumulation of nanosized DDS within solid tumors thanks to fenestrations in the vasculature and a poor lymphatic clearance (5).

Organic synthesis as a discipline has accompanied this evolution of drug delivery into a mature field by providing synthetic tools for the efficient bioconjugation of drugs, polymers, or targeting ligands, and the preparation of novel building blocks and polymeric materials for the construction of DDS with new properties (6). Indeed, chemistry is a central science where phenomena occur and properties are defined at the molecular level. The ability of putting together small building blocks into larger structures has been at the core of evolution and can be used to create novel properties and functions. The level of efficiency achieved by biological systems has inspired chemists in the search for more efficient processes with production of minimal waste. In this way, concepts such as atom economy (every atom of a reagent should be included in the product of the reaction), energy efficiency (the drive to reduce the energy requirements of chemical processes), catalysis (aimed to lower the energy input and avoid the use of stoichiometric reagents), step economy (the drive to reduce the number of synthetic steps), as well as safety and toxicity issues have been adopted in the search of more efficient and environmentally friendly procedures (green chemistry) (7).

In this context, Sharpless and coworkers introduced in 2001 the concept of click chemistry (8), in an effort to focus the attention on the easy production of properties rather than on challenging structures. The idea is to confine the whole range of chemical transformations

\section{Click Chemistry for Drug Delivery Nanosystems}


available to a set of processes with a high thermodynamic driving force, allowing the efficient and easy transformation of "spring-loaded" starting materials into new substances with useful properties.

This Expert Review covers the use of click procedures for the preparation and functionalization of nanoparticulate DDS. Rather than a comprehensive revision of the literature, a selection of -in our view- the most relevant examples reported in the area is presented following a historical perspective. The review begins with a description of the characteristics that define a reaction as click, and the evolution of those criteria when adapted to fields other than those originally considered by Sharpless and coworkers. A critical assessment on the tendency found in the literature to label as click many reactions that do not meet these requirements is also included. As the vast majority of the examples on the application of the click concept to drug delivery nanosystems rely on the $\mathrm{Cu}(\mathrm{I})$-catalyzed azide-alkyne cycloaddition (CuAAC) (9),(10), the following section discusses the advantages and disadvantages of the use of CuAAC in bioconjugation. The need of a correct selection of catalyst, ligand, solvent, and experimental conditions to avoid degradation of the substrates under CuAAC conditions is also addressed. These features are of application not only to bioconjugation but to any other field. After these introductory sections, a selection of literature examples dealing with the use of click reactions [CuAAC, Michael addition, Diels-Alder, thiol-ene coupling (TEC), strain-promoted azide-alkyne cycloaddition (SPAAC)] for the preparation and functionalization of nanosized DDS is included. To facilitate reading, these examples have been organized according to the nature of the nanosystem:

Polymeric Nano- and Microparticulate Delivery Systems Polymeric Micelles

\section{Click Chemistry for Drug Delivery Nanosystems}


Polymeric Nanoparticles (NP)

Polymersomes and Liposomes

Polymeric Capsules and Microspheres

Metal and Silica Nanoparticles

Gold (AuNP) and Noble-Metal Nanoparticles

Magnetic Nanoparticles (MNP)

Quantum Dots (QD)

Silica Nanoparticles (SiNP)

Carbon Nanotubes (CNT) and Fullerenes

Bionanoparticles (BNP)

Keeping in mind the multidisciplinarity of the drug delivery audience, it has been our intention to focus our attention not only on the chemical aspects of the click procedures, but also on the merits of the resulting DDS, paying special emphasis on their usefulness in therapy and diagnosis. Brief comments on the particular advantages of the conjugates produced, drugs being encapsulated, efficiency of the targeting processes, or the existence of in vivo or in vitro assays are also commented. In a complementary following-up review, we will discuss on the use of click chemistry for drug delivery applications with synthetic polymers, dendrimers, and biopolymers.

Click Chemistry for Drug Delivery Nanosystems 


\section{THE CONCEPT OF CLICK CHEMISTRY}

In 2001, Sharpless and coworkers introduced the concept of click chemistry in the field of drug discovery (8). In an effort to focus the attention on the easy production of properties rather than on challenging structures, these authors suggested that: "all searches (of drug candidates) must be restricted to molecules that are easy to make". This strategy was designed to enable the straightforward and economic synthesis of large libraries of new compounds with a minimum synthetic cost. With this aim, it was proposed to confine the whole range of possible chemical transformations to a set of processes with a high thermodynamic driving force (usually higher than $20 \mathrm{Kcal} \cdot \mathrm{mol}^{-1}$ ), which could allow the efficient transformation of "spring-loaded" starting materials into new substances with useful biological properties. In addition, among the highly energetic starting materials available, "those provided by Nature, petroleum chemistry, or easily manufactured from any of those" were selected, being olefins and acetylenes the preferred candidates.

Besides being highly exothermic processes, click reactions should be also characterized by a high orthogonality. This means that coupling partners in click processes should have a wide functional group tolerance, but selectively react to each other under a broad range of experimental conditions. In this way, the need of protecting groups is avoided. Thanks to these intrinsic characteristics, reactivity and orthogonality, click processes are reliable and clean transformations of broad scope, that proceed in quantitative (or near-quantitative) yields, where simple or no purifications are required.

In order to provide an adequate toolbox of chemical transformations, a set of stringent criteria was established for a process to be considered as click (8): "(to) be modular, wide in scope, give very high yields, generate only inoffensive byproducts that can be removed by

\section{Click Chemistry for Drug Delivery Nanosystems}


nonchromatographic methods, and be stereospecific (but not necessarily enantioselective). The required process characteristics include simple reaction conditions (ideally, the process should be insensitive to oxygen and water), readily available starting materials and reagents, the use of no solvent or a solvent that is benign (such as water) or easily removed, and simple product isolation. Purification -if required- must be by nonchromatographic methods, such as crystallization or distillation, and the product must be stable under physiological conditions". These very stringent criteria are actually fulfilled only by a narrow set of chemical transformations, mainly comprising carbon-heteroatom bond forming reactions. Four main groups were originally defined on these basis (8): (i) additions to carbon-carbon multiple bonds (such as epoxidation, dihydroxylation, aziridination, sulfenyl halide addition, and Michael addition chemistry), (ii) nucleophilic substitution chemistry (such as ring-opening reactions of strained cycles, e.g., epoxides, aziridines, aziridinium ions, and episulfonium ions), (iii) cycloadditions of unsaturated species (such as 1,3-dipolar cycloadditions and Diels-Alder reactions), and (iv) carbonyl chemistry of the "non-aldol" type (such as formation of ureas, thioureas, aromatic heterocycles, oxime ethers, hydrazones, and amides). The potential of these four groups of click reactions for the efficient preparation of complex structures has been highlighted by Sharpless and coworkers in short synthetic sequences as seen in Fig. 1 . Interestingly, it is worth to point out that although the concept of click chemistry has emerged recently, it essentially relies on classical chemical transformations, and therefore, publications appearing before the click era (and hence not labeled as click) should be taken into account in this context. An illustrative example is the century-old photochemically/thermally induced radical addition of thiols to alkenes (i.e., thiol-ene coupling, TEC), which has now reappeared as the thio-click reaction.

\section{Click Chemistry for Drug Delivery Nanosystems}




\section{Fig. 1}

Among the click reactions proposed so far, the $\mathrm{Cu}(\mathrm{I})$-catalyzed cycloaddition of azides and terminal alkynes (CuAAC) has attracted special interest (Fig. 2). Independently reported by the groups of Meldal (9), and Fokin and Sharpless (10), this reaction displays one of the greatest orthogonaties ever seen. As opposed to the classical thermal Huisgen 1,3-dipolar azide-alkyne cycloaddition (AAC), where high temperatures $\left(\mathrm{ca} .100^{\circ} \mathrm{C}\right)$ and long reaction times are typically required, the $\mathrm{Cu}(\mathrm{I})$ catalysis significantly reduces the activation barrier, allowing the reaction to proceed with very good rates at room temperature, both in aqueous media and organic solvents (Fig. 2). Moreover, the CuAAC is regioespecific, leading exclusively to 1,4-disusbstituited 1,2,3triazoles, whereas mixtures of regioisomers are obtained under non-catalyzed conditions. In addition, the ease with which azide and alkyne moieties are introduced both chemically (nucleophilic substitution, diazo transfer, incorporated into low molecular weight tags) and biologically (incorporating acetylene and azido amino acids into proteins in either a residuespecific or site-specific fashion) (11), along with their small size and exceptional stability in complex environments, have allowed their incorporation into a vast range of substrates as handles with hidden reactivity. All these features justify $\mathrm{CuAAC}$ at the leading edge within the click reports.

\section{Fig. 2}

Since click chemistry was originally defined by Sharpless and coworkers, an ever increasing number of publications dealing with its application to different areas of research have quickly appeared in the literature. Fields such as polymer and materials science, medicinal chemistry, chemical biology, and the pharmaceutical sciences, among others, have clearly profited from the click concept (12),(13),(14),(15). However, in spite of this favorable reception,

\section{Click Chemistry for Drug Delivery Nanosystems}


a survey of the literature reveals that in many publications, even entitled as "click", some of the fundamental concepts of click chemistry are being misinterpreted. For instance, the use of heating to accelerate the reaction rate has been branded by different authors as against the "simple reaction conditions" requirement. Actually, it should be emphasized that click reactions are generally characterized by having high activation energies, and therefore, they are often performed by heating (8) (Fig. 1). The use of organic solvents has also been controversial, as sometimes it has been stated that click reactions are only allowed to be performed in water, aqueous mixtures, or neat. In this sense, while water is often the preferred reaction medium, organic solvents can be also used as nicely illustrated by Sharpless and coworkers in their seminal report (Fig. 1) (8).

On the other hand, given the original application of click chemistry to drug discovery, it is clear that the stringent criteria for a click process might be revised when adapted to other fields. This adjustment, however, must be in agreement with the click philosophy. For instance, while stereospecificity is demanded for drug discovery, it may not be important for delivery purposes, or in polymer and materials science. In a similar way, substrates such as many DDS cannot be considered as "readily available starting materials", as several steps of "non-click" type chemistry are frequently required for their preparation.

However, besides these particular adjustments, some key requirements of click chemistry are sometimes being severely distorted in papers claimed as click. A good example is the use of chromatographic purifications that clearly violates the click philosophy, and could be only accepted when dealing with macromolecular structures that are difficult to purify by the typical techniques demanded of low molecular weight compounds (e.g, crystallization or distillation).

\section{Click Chemistry for Drug Delivery Nanosystems}


To sum up, in spite CuAAC being considered as "the cream of the crop" among the click reactions, not every single process relying on CuAAC necessarily fulfills the click requisites. In other words, mixing an azide and an alkyne in the presence of $\mathrm{Cu}(\mathrm{I})$ does not necessarily imply a click process if accompanied, for instance, by low yields or tedious chromatographic purifications. Moreover, experimental conditions such as catalyst loading, ratio of reagents, and formation of byproducts should also be considered in evaluating the overall efficiency and convenience of the process.

Finally, we would like to highlight that the high impact of the click concept in chemistry and other disciplines has sometimes derived in an abusive use of the term click. For instance, a number of reactions that strongly defy the click philosophy have been proposed as new examples of click reactions. On the other hand, well-known and established reactions are now being "relabeled" with the fashionable click tag (e.g., thio-click coupling, thio-bromo click, or DielsAlder click reactions). In addition, the relevance of CuAAC among the click reactions has sometimes led to label this as "the click reaction", obviating all other processes fulfilling the click criteria. Readers must be aware of the above considerations and, in order to avoid misinterpretations, critical reading is advised. 


\section{BIOCONJUGATION VIA HUISGEN AZIDE-ALKYNE CYCLOADDITIONS}

Bioconjugation techniques play an important role in drug delivery. The conjugation of peptides, antibodies, or aptamers on the surface of DDS allows their active targeting to specific cells and organs. In addition, the coupling of synthetic polymers to biomolecules has been described as a method to improve their pharmacokinetics and to increase their stability. Other interesting applications of bioconjugation deal with the labeling of biomacromolecular therapeutics such as proteins and nucleic acids, with tags for in vivo imaging purposes. With the aim of preserving the structural and functional integrity of biomolecules, bioconjugation processes should be ideally performed with fast kinetics, and under mild conditions in aqueous media.

Thanks to its high orthogonality, reliability, and experimental simplicity for nonspecialists, it is not surprising that CuAAC quickly found application in the bioconjugation arena soon after being described in the literature. Unfortunately however, the use of CuAAC in bioconjugation has not been a straightforward task. Thus, the required $\mathrm{Cu}(\mathrm{I})$ catalyst has been demonstrated to induce severe structural damage to biomolecules. Also, under the original conditions reported by Fokin, Sharpless, and coworkers $\left(\mathrm{CuSO}_{4} /\right.$ sodium ascorbate) (8), CuAAC often lacks adequate kinetics at the micromolar range typically employed in bioconjugation. Taking into account the relevance of CuAAC within click chemistry, it is the intention of this section to analyze the encountered problems and proposed solutions for the efficient and harmless conjugation of biomolecules via CuAAC.

Some of the limitations of CuAAC in bioconjugation were soon pointed out by Fokin, Sharpless, Finn, and coworkers who described that cowpea mosaic virus (CPMV) resulted either degraded or aggregated in the presence of $\mathrm{Cu}$ and the reducing agents used for the in situ

\section{Click Chemistry for Drug Delivery Nanosystems}


generation of $\mathrm{Cu}(\mathrm{I})(16)$. Indeed, the role of $\mathrm{Cu}$ in the oxidative stress of biomolecules is wellknown. $\mathrm{Cu}$ ions can easily promote the generation of reactive oxygen species (ROS), such as $\mathrm{H}_{2} \mathrm{O}_{2}$, and the hydroxyl ( $\left.{ }^{\circ} \mathrm{OH}\right)$ and superoxide $\left(\mathrm{O}_{2}{ }^{\circ-}\right)$ radicals, which are responsible of biological damage (17). The production of ${ }^{\circ} \mathrm{OH}$, the most powerful ROS species, is mediated by a Fenton reaction involving a transition metal, $\mathrm{Cu}(\mathrm{I})$ in this case, and $\mathrm{H}_{2} \mathrm{O}_{2}$ (eq 1) (18). The required $\mathrm{H}_{2} \mathrm{O}_{2}$ is formed in situ via two possible mechanisms: (i) in the presence of ascorbate $\left(\mathrm{AH}_{2}\right), \mathrm{O}_{2}$ can be reduced to $\mathrm{H}_{2} \mathrm{O}_{2}$ in a process catalyzed by traces of $\mathrm{Cu}$ (II) or other transition metal ions (eq 2) (18); (ii) in the absence of ascorbate or other reducing agents, $\mathrm{Cu}(\mathrm{I})$ itself can reduce $\mathrm{O}_{2}$ to $\mathrm{H}_{2} \mathrm{O}_{2}$ through a two-step process involving the mediation of $\mathrm{O}_{2}{ }^{--}($eq 3,4) (17). The presence of ROS during the functionalization of biomolecules, such as proteins, nucleic acids, polysaccharides, and lipids is therefore a major concern, as the structural and functional integrity of these substrates might result severely compromised.

$$
\begin{aligned}
& \mathrm{Cu}(\mathrm{I})+\mathrm{H}_{2} \mathrm{O}_{2} \rightarrow \mathrm{Cu}(\mathrm{II})+{ }^{\circ} \mathrm{OH}+\mathrm{OH}^{-}(1) \\
& \mathrm{AH}_{2}+\mathrm{O}_{2} \rightarrow \mathrm{A}+\mathrm{H}_{2} \mathrm{O}_{2}(2) \\
& \mathrm{Cu}(\mathrm{I})+\mathrm{O}_{2} \rightarrow \mathrm{O}_{2}{ }^{\cdot-}+\mathrm{Cu}(\mathrm{II})(3) \\
& \mathrm{Cu}(\mathrm{I})+\mathrm{O}_{2}{ }^{-}+2 \mathrm{H}^{+} \rightarrow \mathrm{Cu}(\mathrm{II})+\mathrm{H}_{2} \mathrm{O}_{2}
\end{aligned}
$$

Other undesired reactions, such as the homocoupli

ng of terminal alkynes through $\mathrm{Cu}(\mathrm{I})$-catalyzed Glaser reaction, have been also observed under $\mathrm{CuAAC}$ conditions (19). Altogether, these secondary processes diminish the efficiency of the conjugation, and are particularly undesired in the functionalization of macromolecular platforms, as purifications are often eventually impossible.

\section{Click Chemistry for Drug Delivery Nanosystems}


In a general sense, the aforementioned drawbacks of CuAAC can be efficiently overcome with the proper selection of the catalytic system, which usually requires the use of a $\mathrm{Cu}(\mathrm{I})$ chelating ligand (20),(21). These ligands are designed to: (i) stabilize the $\mathrm{Cu}(\mathrm{I})$ oxidation state, (ii) accelerate the cycloaddition reaction, (iii) prevent the formation of undesired byproducts, and (iv) sequester $\mathrm{Cu}$ ions to reduce the structural damage to biomolecules and to facilitate the purification process. In this regard, tris(benzyltriazolylmethyl)amine (TBTA) (20), tris(hydroxypropyltriazolylmethyl)amine (THPTA) (20), and bathophenanthroline disulphonated disodium salt (BPDS) (21) have been the ligands most usually employed in bioconjugation (Fig. $3)$.

\section{Fig. 3}

The tetradentate binding ability of tris(triazolylmethyl)amine ligands allows the formation of stable $\mathrm{Cu}(\mathrm{I})$ chelates that account for an increase of $c a .300 \mathrm{mV}$ in the redox potential of $\mathrm{Cu}(\mathrm{I}) / \mathrm{Cu}(\mathrm{II})$ (20). TBTA was the first member of this family to be identified. The superior protection conferred by TBTA to $\mathrm{Cu}(\mathrm{I})$ towards air oxidation renders unnecessary the exclusion of $\mathrm{O}_{2}$ from the reaction medium. Nevertheless, a great shortcoming of TBTA is its poor solubility in water, which has fuelled the development of water-soluble analogs, such as THPTA (22). In addition, partly because of this poor water solubility, the typical rate enhancement of TBTA has been claimed as low, with kinetics comparable to other classical bioconjugation reactions.

BPDS represents an attractive alternative to tris(triazolylmethyl)amine ligands. It exhibits a high solubility in water and an excellent catalytic activity, even under high dilution conditions or when working with small excess of coupling probes (23). Conversely, the BPDS/Cu(I) catalytic system has revealed very sensitive to air oxidation, and unless a sacrificial reducing agent is

\section{Click Chemistry for Drug Delivery Nanosystems}


added, the rigorous exclusion of $\mathrm{O}_{2}$ from the reaction medium is required to avoid oxidation of $\mathrm{Cu}(\mathrm{I})$.

A survey of the literature reveals many examples to illustrate that the correct selection of the catalytic system allows CuAAC to become a safe and efficient tool in bioconjugation. However, despite the use of $\mathrm{Cu}(\mathrm{I})$-chelating ligands, a loss of bioactivity/integrity of proteins and nucleic acids during CuAAC bioconjugation has been reported in several cases (24), which highlight the necessity of more convenient $\mathrm{CuAAC}$ protocols. To this end, Finn and coworkers have reported an electrochemically protected version of the $\mathrm{CuAAC}$, where $\mathrm{Cu}$ (II) is electrochemically reduced to $\mathrm{Cu}(\mathrm{I})$ in the presence of the desired accelerating ligand (25) (Fig. 4). In this way, the oxidation state of $\mathrm{Cu}(\mathrm{I})$ is continuously preserved without the addition of any sacrificial reducing agent. In addition, oxidative degradation of substrates by ROS is drastically diminished, as the $\mathrm{O}_{2}$ present in the reaction medium, which is necessary for the generation of ROS (eq 1-4), is electrochemically reduced to $\mathrm{H}_{2} \mathrm{O}$ (i.e., $\mathrm{O}_{2}+4 \mathrm{H}^{+}+4 \mathrm{e}^{-} \rightarrow 2 \mathrm{H}_{2} \mathrm{O}$ ). In a more recent report, this group has proposed a bioconjugation protocol relying on the simplicity of the $\mathrm{Cu}(\mathrm{II}) /$ ascorbate system and the use of THPTA (22) (Fig. 4). Besides the typical ligandaccelerating effect, THPTA was found to mediate the decomposition of $\mathrm{H}_{2} \mathrm{O}_{2}$ produced in the reaction medium and to act as a radical scavenger. As previously pointed out by Brown and coworkers (26), a 5-fold excess of THPTA vs $\mathrm{Cu}(\mathrm{I})$ was recommended to minimize the oxidative degradation of substrates. The addition of aminoguanidine as carbonyl capturing reagent was also recommended to preserve substrates from reaction with ascorbate and other byproducts derived from ascorbate oxidation (Fig. 4).

Fig. 4 
Alternatively, more benign $\mathrm{Cu}$-free $\mathrm{AAC}$ bioconjugation strategies, not requiring the addition of cytotoxic metals and additives, have also appeared. The group of Bertozzi has taken advantage of the inherent ring strain of cyclooctynes as an effective way for lowering the activation barrier of AAC (24) (Fig. 5). This strain-promoted AAC variant named as SPAAC has found widespread application in the context of the bioorthogonal chemical reporter strategy for the study of dynamic processes of biomolecules. In addition, SPAAC has recently gained significance in polymer/materials science and drug delivery applications, where the presence of $\mathrm{Cu}$ prevents the use of CuAAC (27),(11). Rate optimization of SPAAC for faster bioconjugation under mild conditions has been reported by the groups of Bertozzi, Boons, and van Hest and van Delft, by means of difluorocyclooctyne (DIFO) (28),(29), dibenzocyclooctyne (DIBO) (30), dibenzoazacyclooctyne (DIBAC) (31), biarylazacyclooctynone (BARAC) (32), and bicyclononyne (BCN) (33) derivatives (Fig. 5). The higher reactivity of these reagents compared to unfunctionalized cyclooctyne relates to the presence of electron-withdrawing groups and increased ring strain, which have afforded reaction rate values comparable to those of ligand-less CuAAC. Interestingly, a cyclooctyne precursor of the reactive DIBO with the triple bond masked as cyclopropenone has been developed by Boons, Popik, and coworkers for the photo-triggering of SPAAC under UV irradiation (ca. $350 \mathrm{~nm}$ ) (34) (Fig. 5).

\section{Fig. 5}

It must be pointed out that although the use of cyclooctynes hampers SPAAC to qualify as a pure click reaction as originally defined (lack of regioselectivity and no readily available starting materials), it essentially matches the click philosophy when dealing with macromolecular structures as those in drug delivery applications.

\section{Click Chemistry for Drug Delivery Nanosystems}




\section{POLYMERIC NANO- AND MICROPARTICULATE DELIVERY SYSTEMS}

Over the past few decades, there has been considerable interest in the use of delivery systems for the safe and efficient administration of drugs (1). With this aim, various polymeric nano- and microparticulate systems have been described, including polymeric micelles and NP, polymersomes, and polymeric capsules and microspheres (2). These DDS have been reported to effectively deliver drugs to target sites and hence to increase the therapeutic index, while reducing undesirable side effects and the frequency of administration. In particular, DDS can enhance the therapeutic activity by prolonging the drug half-life, improving the solubility of hydrophobic drugs, reducing the immunogenicity, and allowing a sustained or stimuli-triggered release of drugs (1),(35). In this section the use of click chemistries for the preparation and functionalization of different polymeric nano- and microparticulate delivery systems is discussed.

\section{Polymeric Micelles}

Polymeric micelles are spherical nanostructures usually obtained by self-assembly of amphiphilic block copolymers in water, that are considered as promising drug carriers (36). They are characterized by a size in the range $20-100 \mathrm{~nm}$ (similar to that of lipoproteins and viruses) and a core-shell structure consisting of a hydrophobic core surrounded by a hydrophilic shell. While the core provides a potential reservoir for hydrophobic drugs, the hydrophilic shell ensures low protein adsorption, improved drug solubility, and long circulation times in the blood stream. This stealth property, along with their characteristic small sizes, allow the selective accumulation of polymeric micelles in tumor tissues due to the known EPR effect (5).

\section{Click Chemistry for Drug Delivery Nanosystems}


Various click reactions, especially $\mathrm{CuAAC}$, have been used in either the preparation of block copolymers that self-assemble into micelles, or in the surface functionalization of polymeric micelles. As an example of the first category, Sumerlin and coworkers used CuAAC for the functionalization of a temperature-responsive block copolymer with folic acid (FA) (37). A thermoresponsive azido-terminated $\operatorname{poly}(N, N$-dimethylacrylamide $)-b$-poly $(N$ isopropylacrylamide) (N3-PDMA- $b$-PNIPAM) was synthesized by reversible additionfragmentation chain transfer (RAFT) polymerization using an azido-functionalized chain transfer agent (CTA), NIPAM and DMA, which was subsequently decorated with a propargyl folate by CuAAC (CuBr, PMDETA, DMF). The resulting block copolymer underwent self-assembly to yield polymeric micelles of approximately $46 \mathrm{~nm}$ at $34{ }^{\circ} \mathrm{C}$, as potential drug delivery systems with active-targeting for cancer tissues.

Following a similar approach, the groups of Li (38) and Stenzel (39) independently prepared block copolymers incorporating pendant azides/alkenes in one of the blocks that were subsequently functionalized with carbohydrates by means of CuAAC and TEC to give amphiphilic block copolymers. Li and coworkers prepared a series of biodegradable glycopolymers based on poly( $\varepsilon$-caprolactone) (PCL). PCL- $b$-PBrCL was synthesized by ringopening polymerization and then, the bromine groups were transformed into azides and subsequently functionalized with alkynyl saccharides via $\mathrm{CuAAC}(\mathrm{CuBr}$, PMDETA, DMF) to give amphiphilic block copolymers that self-assembled into micellar aggregates (38).

Taking advantage of TEC, Stenzel and coworkers prepared glycomicelles as potential drug carriers. A block copolymer containing di(ethylene glycol) methyl ether methacrylate and 2hydroxyethyl methacrylate (HEMA) was prepared by RAFT polymerization, and further modified with glucothiose via alkene incorporation and TEC (UV light, 2,2-dimethoxy-2-phenyl

\section{Click Chemistry for Drug Delivery Nanosystems}


acetophenone, DMF). The resulting glycosylated block copolymer led to the formation of thermoresponsive micelles (39) (Fig. 6).

Fig. 6

Several interesting examples on the use of CuAAC for the preparation of PEG-dendritic block copolymer-based micelles and other nanostructures have been reported by the group of Dong $(40,41,42)$. For instance, they prepared spherical flower-like micelles of $c a .50 \mathrm{~nm}$ by self-assembly in aqueous solution of dendron-like PCL- $b$-PEG- $b$-dendron-like PCL triblock copolymers prepared by $\mathrm{CuAAC}(\mathrm{CuBr}$, PMDETA, DMF) (Fig. 7). These micelles were able to encapsulate the anticancer drug doxorubicin (DOX) with high loading efficiency, and displayed a sustained release profile.

Fig. 7

Recently, Kakkar, Maysinger, and coworkers reported the preparation of miktoarm micelles as nanocarriers for nimodipine, a hydrophobic drug employed in the prevention and treatment of delayed ischemic neurological disorders (43). In their approach, miktoarm $\mathrm{A}_{2} \mathrm{~B}$ polymers $(\mathrm{A}=\mathrm{PEG}, \mathrm{B}=\mathrm{PCL})$ were constructed from a core containing one hydroxyl and two alkynyl groups, which allowed the incorporatiom of two $\mathrm{PEG}_{3} \mathrm{~N}_{3}$ chains by $\mathrm{CuAAC}(\mathrm{CuBr}$, PMDETA, degassed DMF), followed by ring-opening polymerization of $\varepsilon$-caprolactone. After self-assembly of the miktoarm polymers into spherical micelles, nimodipine was loaded with high encapsulation efficiency. In vitro studies revealed a slow release which results advantageous in order to reduce the frequency of administration and toxicity.

Zhang and coworkers have also employed CuAAC for the preparation of core-shell micelles with tumor-triggered targeting properties (44) (Fig. 8). In their approach, an azidomodified $\alpha$-cyclodextrin (CD), designed to act as a host for the terminal phenyl group at the

\section{Click Chemistry for Drug Delivery Nanosystems}


hydrophilic polymer P(NIPAM-co-N-acroyloxysuccinimide), was coupled via $\mathrm{CuAAC}\left(\mathrm{CuSO}_{4}\right.$, sodium ascorbate, PMDETA) to an alkynated $\beta$-CD, designed to complex hydrophobic PCL bearing a terminal adamantyl group. The $\mathrm{CD}$ dimer was used to connect the hydrophilic and hydrophobic segments that subsequently assembled. The resulting micelles were further functionalized with an Arg-Gly-Asp (RGD) peptide as targeting ligand to improve the cellular uptake efficacy. PEGylation via $\mathrm{pH}$-sensitive imine bonds was employed to protect the peptide ligands in normal tissues and body fluids. In addition, DOX was loaded into the micelles and two dyes were conjugated to the polymer components to track the formation of the micelles. The authors demonstrated that the targeting property of the micelles was switched on only after removing the PEG segment at the tumor site, which also triggered the release of DOX by decreasing the lower critical solution temperature of the micelles.

\section{Fig. 8}

$\mathrm{CuAAC}$ has also been employed for the surface functionalization of polymeric micelles. In one of the first examples, reported by Lutz and coworkers, the in situ shell-functionalization of biocompatible micelles with a hydrophobic core and a PEG-based corona was described (45). Surfactants composed of a hydrophobic cholesterol moiety and a hydrophilic polymer segment were synthesized from various oligo(ethylene glycol) methacrylates by atom transfer radical polymerization (ATRP), initiated by cholesteryl-2-bromoisobutyrate. After polymerization, the bromine chain-ends were transformed into azides. Some of the resulting polymers formed thermoresponsive micellar aggregates $(c a .100 \mathrm{~nm})$ in aqueous solution that could be decorated with propargyl alcohol as a model ligand by $\mathrm{CuAAC}\left(\mathrm{CuSO}_{4}\right.$, sodium ascorbate).

Poly(alkyl cyanocrylate) (PACA) is a biodegradable polymer well-known in the pharmaceutical field, that has been developed for more than two decades by the group of

\section{Click Chemistry for Drug Delivery Nanosystems}


Couvreur for the preparation of different drug delivery nanostructures (46). Nicolas and coworkers have recently reported the preparation of clickable PEGylated PACA micelles from a poly[(hexadecyl cyanoacrylate)-co-azidopoly(ethylene glycol) cyanoacrylate] copolymer, displaying azide functionalities at the end of the PEG chains (47) (Fig. 9). CuAAC functionalization was either performed directly on the polymer (CuBr, PMDETA, DMF) followed by self-assembly in aqueous solution, or alternatively onto the surface of preformed micelles in aqueous media $\left(\mathrm{CuSO}_{4}\right.$, sodium ascorbate). This versatile approach opens the door to ligand-functionalized biodegradable PACA micelles with stealth properties for biomedical applications.

\section{Fig. 9}

The delivery of ophthalmic drugs using polymeric micelles with targeting RGD peptides is a promising tool for the treatment of a wide range of corneal epithelial defects. With this aim, Shoichet and coworkers have designed peptide-modified micelles by taking advantage of a CuAAC surface decoration (48). An amphiphilic block copolymer was prepared from poly(2methyl-2-carboxytrimethylene carbonate-co-D,L-lactide) [P(TMCC-co-LA)] and an amineterminated PEG-N 3 . The resulting $\mathrm{P}(\mathrm{TMCC}-\mathrm{co}-\mathrm{LA})-g-\mathrm{PEG}-\mathrm{N}_{3}$ copolymer self-assembled in aqueous solution and the azide groups on the surface of the micelles were functionalized with alkyne-modified KGRGDS peptides via $\mathrm{CuAAC}$ in water $\left(\mathrm{CuSO}_{4}\right.$, sodium ascorbate). The covalently bound RGD peptides maintained their bioactivity and binding affinity towards rabbit corneal epithelial cells.

The group of Shoichet has also described an elegant route to immuno-polymeric micelles based on Diels-Alder cycloaddition (49) (Fig. 10). An amphiphilic P(TMCC-co-LA)-g-PEGfuran carrying furan as a terminal diene was prepared and self-assembled. The furan groups on

\section{Click Chemistry for Drug Delivery Nanosystems}


the outer PEG shell were then reacted with a maleimide-modified anti-HER2, a therapeutic antibody used to treat breast cancer. Under these mild conditions, the classical low efficiency and loss of activity associated with the coupling of sensitive antibodies were overcome. In addition, the procedure takes advantage of the increased reaction rate and stereoselectivity of Diels-Alder couplings in aqueous media. The anti-HER2 immuno-micelles specifically bound to HER2overexpressing cells, demonstrating the strength of this procedure to create bioactive immunomicelles. As only a few anti-HER2 antibodies on the surface of the micelles were necessary for targeting, thousands of PEG-furan chains were available for coupling to a DOX-maleimide conjugate in a subsequent step (50). The resulting DOX-conjugated immuno-micelles represent an entirely new method for localized codelivery of chemotherapeutics and antibodies.

\section{Fig. 10}

The Michael addition of thiols to activated olefins has been successfully applied to the functionalization of micelles. In an interesting example, the groups of An and Stucky have reported a combination of Michael addition and $\mathrm{CuAAC}$ for the orthogonal functionalization of the core and shell of polymeric micelles (51) (Fig. 11). Monodisperse core-shell micelles (65-95 $\mathrm{nm}$ ) were prepared by a RAFT-mediated precipitation polymerization of NIPAM, using a preformed PDMA RAFT polymer as CTA. The PNIPAM generated during the process constituted the core in which trithiocarbonate groups were located, while PDMA blocks carrying azido groups formed the shell on the surface. The core of the micelle could be functionalized with a fluorescein $o$-acrylate through a one-pot aminolisis/Michael addition, while the shell was decorated with a dansyl probe via $\mathrm{CuAAC}\left(\mathrm{CuSO}_{4}\right.$, sodium ascorbate, DMF- $\left.\mathrm{H}_{2} \mathrm{O}\right)$.

\section{Fig. 11}


The group of Nguyen has shown that drug-containing (indomethacin or DOX) amphiphilic polynorbornenes can self-assemble into therapeutically active core-shell polymer micelles with uniform, tunable diameters (52),(53). More recently, the same group has reported a CuAAC strategy for the functionalization of these micelles with various bioactive ligands/particles (54). An indomethacin-containing norbornene monomer and a trimethylsilyl (TMS) protected acetylene-terminated hydrophilic hexa(ethylene) glycol norbornene monomer were sequentially polymerized in different ratios by ring-opening metathesis polymerization. After deprotection of the TMS groups, block copolymers self-assembled in water into alkyne-decorated NP that were decorated by $\mathrm{CuAAC}\left(\mathrm{CuSO}_{4}\right.$, sodium ascorbate) with azido-functionalized folate, biotin, or AuNP.

Jing, Chen, and coworkers have reported on the surface functionalization of microspheres (55) and biodegradable polymer fibers (56) with proteins and antibodies by means of CuAAC. In a recent paper, an artificial oxygen carrier was constructed by conjugating hemoglobin to biodegradable micelles (57) (Fig. 12). The authors prepared a series of triblock copolymers from PLA, poly(2-methyl-2-carboxyl-propylene carbonate) (PMPC), and PEG, carrying pendant propargyl groups at the PMPC middle block. These amphiphilic copolymers self-assembled in aqueous solution into core-shell micelles. Hemoglobin containing azide groups was conjugated via $\mathrm{CuAAC}\left(\mathrm{CuSO}_{4}\right.$, sodium ascorbate, histidine) to the propargyl groups on the core surface, a privileged location to facilitate coupling while ensuring protection of the protein against the immunological system by the PEG corona. The hemoglobin content and the oxygen binding ability of the micelles were studied, revealing a maximum hemoglobin loading of $70 \mathrm{wt} \%$ and retention of its oxygen-binding ability.

\section{Fig. 12}

\section{Click Chemistry for Drug Delivery Nanosystems}


Quite similarly, Liu, Zhao, and coworkers reported the preparation of an azido-containing amphiphilic triblock copolymer PEG- $b$-poly(azidoethyl methacrylate)- $b$-poly(methyl methacrylate) that in aqueous media self-assembled into spherical micelles carrying azide groups at the hydrophobic/hydrophilic interface. The conjugation of biotin via $\mathrm{CuAAC}\left(\mathrm{CuSO}_{4}\right.$, sodium ascorbate, $t-\mathrm{BuOH}-\mathrm{H}_{2} \mathrm{O}$ ) resulted in the formation of a functional interface between the hydrophilic shell and the hydrophobic core with demonstrated bioavailability (58).

An interesting family of polymeric micelles is the so-called Polyion Complex (PIC). Originally described by the groups of Kataoka (59) and Kabanov (60), PIC micelles are formed by electrostatic interaction between oppositely charged polymers. Their electrical neutrality, small size, and fairly narrow size distribution makes them very appealing for drug delivery purposes. The group of Fernandez-Megia and Riguera recently described the CuAAC $\left(\mathrm{CuSO}_{4}\right.$, sodium ascorbate, $t-\mathrm{BuOH}-\mathrm{H}_{2} \mathrm{O}$ ) functionalization of PEG-dendritic block copolymers of the GATG (gallic acid-triethylene glycol) family with peripheral anionic groups. One of the copolymers carrying 27 peripheral sulphates led to PIC micelles of $c a .25 \mathrm{~nm}$ by incubation with poly-L-lysine (PLL) (Fig. 13). These micelles resulted to be remarkably stable under physiological conditions $\left(150 \mathrm{mM} \mathrm{NaCl}, 37^{\circ} \mathrm{C}\right)$, high ionic strengths, and even freeze dryingresuspension, as a result of an increased stability granted by the dendritic scaffold (61).

\section{Fig. 13}

Liu and coworkers have recently reported another example of $\mathrm{CuAAC}$ in the preparation of PIC micelles (62). A combination of ATRP and CuAAC (CuBr, DMF) was used to obtain two oppositely charged graft ionomers: PNIPAM-grafted poly[methacrylic acid-co-(3-azidopropyl methacrylate $)] \quad\left[\mathrm{P}\left(\mathrm{MAA}-\mathrm{co}-\mathrm{N}_{3} \mathrm{PrMA}\right)\right], \quad$ and $\quad$ PNIPAM-grafted poly $\{[2-$ (trimethylammonium)ethyl methacrylate]-co- $\left.\mathrm{N}_{3} \operatorname{PrMA}\right\}$. The incubation of both ionomers in

\section{Click Chemistry for Drug Delivery Nanosystems}


aqueous solution led to the formation of PIC micelles with thermoresponsive PNIPAM coronas. In a further step, $\mathrm{CuAAC}\left(\mathrm{CuSO}_{4}\right.$, sodium ascorbate) was used again to cross-link the core of the micelles, following a strategy previously reported by the groups of Wooley and Hawker (vide infra), to ensure their stability against ionic strength and $\mathrm{pH}$ changes.

\section{Polymeric Nanoparticles}

In spite of the favorable features of polymeric micelles for drug delivery applications, these assemblies may suffer from spontaneous dissociation at concentrations below their critical micelle concentration (CMC), and from low stability at high temperatures (and ionic strengths in the case of PIC micelles). A common strategy to confer greater stability to these self-assembled structures is the cross-linking of the individual core/shell components to give core cross-linked or shell cross-linked NP $(63,64)$. Wooley, Hawker, and coworkers described the first applications of $\mathrm{CuAAC}$ in this field in a series of very exciting contributions (65),(66). In their first report, an amphiphilic diblock copolymer, poly(acrylic acid)-b-poly(styrene) (PAA- $b$-PS), that had been assembled into micelles and partially functionalized throughout the corona with alkynyl groups, was shell cross-linked with a first generation azido-terminated dendrimer by $\mathrm{CuAAC}\left(\mathrm{CuSO}_{4}\right.$, sodium ascorbate) (65) (Fig. 14). The resulting NP benefited from increased stability and the presence of imbedded azides into the shell for further functionalization. The complementary approach for the preparation of well-defined core cross-linked polymeric NP was realized from an amphiphilic diblock copolymer PAA- $b$-PS, containing alkynyl groups partially incorporated throughout the hydrophobic PS block (66)

Fig. 14.

Click Chemistry for Drug Delivery Nanosystems 
Stabilization of block copolymer micelles following similar CuAAC cross-linking approaches has been extensively investigated by different groups in the last few years. For instance, Liu and coworkers described the preparation of core cross-linked NP with thermoresponsive cores from PDMA- $b$-P(NIPAM-co-azido-propylacrylamide) (67). The same authors in collaboration with the group of Narain have reported the fabrication of two crosslinked micelles with inverted structures from a single water-soluble triblock copolymer composed by poly[2-(2-methoxyethoxy)ethyl methacrylate], PDMA, and poly[2(diethylamino)ethyl methacrylate] blocks (68).

An interesting application of this cross-linking strategy has been developed by Stenzel's group for the preparation of carriers for the delivery of cobalt pharmaceuticals (69). A core-shell micelle was prepared by self-assembly of a poly(trimethylsilyl propargyl methacrylate)- $b$ poly[poly(ethylene glycol) methyl ether methacrylate] obtained by RAFT polymerization, after removal of the TMS protecting groups. Then, the pendant alkyne groups at the core were use as reactive functional groups for cross-linking via $\mathrm{CuAAC}\left(\mathrm{Cu}\right.$ wire, $\mathrm{Cu}\left(\mathrm{PPh}_{3}\right)_{3} \mathrm{Br}$, DIPEA, THF$\mathrm{H}_{2} \mathrm{O}$ ), and as ligands for $\mathrm{Co}_{2}(\mathrm{CO})_{8}$ to generate a macromolecular carrier of the antitumor agents based on (alkyne) $\mathrm{Co}_{2}(\mathrm{CO})_{6}$. It is worth to note that while Co-loaded micelles resulted highly toxic to L929 fibroblast cells, the permanent encapsulation of the Co complex in the cross-linked NP was shown to reduce the toxicity.

Click procedures have been applied not only to the covalent cross-linking of micelles, but also to the surface decoration of NP. In this regard, the group of Fernandez-Megia and Riguera has described the use of SPAAC as an efficient alternative to CuAAC for the orthogonal functionalization of polymeric NP, that avoids the detrimental effects associated to the $\mathrm{Cu}(\mathrm{I})$ catalyst (70) (Fig. 15). In their work towards the CuAAC functionalization of chitosan-g-PEG-N 3

\section{Click Chemistry for Drug Delivery Nanosystems}


NP as carriers across the blood-brain barrier, a severe oxidative depolymerization of the chitosan backbone mediated by ${ }^{\circ} \mathrm{OH}$ was observed, along with high contents of cytotoxic $\mathrm{Cu}$ severely compromising their biomedical applications. These detrimental effects, seen also in other polysaccharides (e.g., dextran and hyaluronic acid), led the authors to explore the use of SPAAC as a safer $\mathrm{Cu}$-free coupling technology. In this way, the surface functionalization of cross-linked chitosan- $g$-PEG-N 3 NP with a cyclooctyne-derived anti-BSA antibody proceeded safely and quantitatively under physiological conditions, in what represents a step forward in the development of environmentally friendly bioconjugation technologies for the preparation of immuno-NP.

\section{Fig. 15}

\section{Polymersomes and Liposomes}

Liposomes are self-closed vesicular structures composed of (phospho)lipid bilayers that have attracted considerable attention in drug/gene delivery and diagnosis, and have reached clinical application especially for the treatment of cancer and opportunistic diseases (71). Although liposomes are not polymeric DDS, they are included in this section due to their relevance in drug delivery and their similarity to polymersomes.

The functionalization of the surface of liposomes with different ligands to target specific cells has been traditionally performed by means of active esters, Michael additions, or hydrazones. The first example on the use of CuAAC with this purpose was reported by the group of Schuber $\left(\mathrm{CuSO}_{4}\right.$, sodium ascorbate, BPDS) who prepared mannosylated vesicles from liposomes incorporating a synthetic lipid carrying a terminal alkyne (72). Interestingly, the use BPDS as $\mathrm{Cu}(\mathrm{I})$-chelating ligand (2-fold excess over $\mathrm{Cu}$ ) allowed a large increase in the yield of mannosylation and a decrease in the reaction time. In a similar manner, Kros and coworkers 
described the surface functionalization of liposomes with a fluorescent probe (73). By means of fluorescence resonance energy transfer (FRET) studies, these authors revealed that the CuAAC functionalization occurred on the liposome surface. More recently, Cai and coworkers optimized the CuAAC conditions for the functionalization of liposomes (74). These authors prepared physically robust clickable polymerized liposomes based on polydiacetylene lipids that were efficiently decorated with multiple functionalities (FITC, coumarin, and a GRGD peptide) without decomposition of the liposomes, a feature usually observed for unsaturated liposomes under $\mathrm{CuAAC}$ conditions. The improved reaction conditions are based on the use of a $\mathrm{Cu}(\mathrm{I})-$ chelating ligand [a tris(triazolylmethyl)amine derivative bearing tetra(ethylenglycol) side chains] that is highly soluble in water, insensitive to air, and has shown to shorten reaction times with reduced amounts of $\mathrm{CuSO}_{4}$ and sodium ascorbate.

Similarly to liposomes, polymersomes have attracted a great deal of attention in the field of drug delivery. They are hollow vesicles characterized by a bilayer membrane built from amphiphilic block copolymers that have many similarities to liposomes, but a greater stability (lower CMC values) and decreased permeability. Since they were reported by the group of Discher and Hammer (75), polymersomes have been the subject of intensive research with applications ranging from imaging agents and delivery vehicles to nanoreactors (76).

The first application of CuAAC to the functionalization of polymersomes was reported by the group of van Hest (77) (Fig. 16). These authors reported the preparation of azido-decorated polymersomes and their conjugation to bioactive ligands, such as a dansyl probe, biotin, and the enhanced green fluorescent protein (EGFP). With this aim, an amphiphilic PS- $b$-PAA was prepared by ATRP, and the terminal bromide end groups were substituted for azides. The resulting copolymers were allowed to self-assemble into vesicular aggregates that were

\section{Click Chemistry for Drug Delivery Nanosystems}


incubated with aqueous solutions of alkyne-functionalized ligands under standard CuAAC conditions $\left(\mathrm{CuSO}_{4}\right.$, sodium ascorbate, TBTA or BPDS). Average functionalization of peripheral azides in the range $40-50 \%$ was estimated by fluorescence measurements. Interestingly, no change in the vesicles morphology was observed during the CuAAC functionalization process.

\section{Fig. 16}

In an interesting contribution, Gillies and coworkers reported a similar method for the attachment of dendrons onto polymer vesicles directed to enhance the availability of peripheral ligands for multivalent interaction with biological targets (78) (Fig. 17). Vesicles were prepared by self-assembly of amphiphilic poly(butadiene)- $b$-poly(ethylene oxide) (PBD- $b$-PEO) and PBD- $b-\mathrm{PEO}-\mathrm{N}_{3}$, mixed in different ratios. The functionalization of these vesicles with a polyester dendritic scaffold incorporating an alkyne focal point was studied by $\mathrm{CuAAC}\left(\mathrm{CuSO}_{4}\right.$, sodium ascorbate, BPDS). Well dispersed vesicles were observed after $\mathrm{CuAAC}$ functionalization when containing up to $20 \%$ of PBD- $b-\mathrm{PEO}-\mathrm{N}_{3}$. Under these conditions, full surface decoration resulted. In a more recent report, the same group has demonstrated that vesicles functionalized with dendritic mannose $\left(\mathrm{CuSO}_{4}\right.$, sodium ascorbate, BPDS) show an enhanced affinity (of 1-2 orders of magnitude) towards lectin Concanavalin A (hemagglutination assay), when compared to vesicles containing an equal density of non-dendritic mannose (similar results with dextrancoated iron oxide NP) (79). This binding enhancement has been attributed to the ability of the dendrons to effectively display ligands and overcome the steric inhibition by surrounding polymer chains, which represents an interesting example of the relevance of the ligand presentation on the binding to biological targets.

Fig. 17 
The group of O'Halloran and Nguyen has described a drop-in procedure for the preparation of polymer-caged nanobins $(\mathrm{PCN})$ from preformed liposomes and a cholesterolterminated PAA (synthesized by nitroxide-mediated controlled radical polymerization), followed by shell cross-linking (80). PCN surpass some of the problems associated with classical liposomes composed of $\mathrm{pH}$-sensitive, amine-modified lipids, and represent a more robust delivery platform with $\mathrm{pH}$-responsiveness under acidic conditions. In a recent report, the same group has described the preparation of clickable PCN from DOX-loaded liposomes and a cholesterol-terminated PAA by covalent cross-linking with an alkyne-modified diamine (81) (Fig. 18). The resulting alkyne-functionalized PCN (ca. $125 \mathrm{~nm})$ were decorated with folic acid via $\mathrm{CuAAC}\left(\mathrm{CuSO}_{4}\right.$, sodium ascorbate) to give folate-targeted DOX-loaded PCN with demonstrated enhanced potency towards folate receptor (FR) positive tumor cells (KB and OvCa432), when compared to the untargeted agent. In addition, the folate-targeted PCN could discriminate FR positive tumor cells as a function of the level of cellular FR expression, showing different degrees of potentiation.

\section{Fig. 18}

\section{Polymeric Capsules and Microspheres.}

Polymer capsules are hollow spherical systems envisioned as promising materials with application for the controlled release of functional molecules, such as drugs or enzymes. They are usually prepared by layer-by-layer (LbL) assembly techniques comprising the deposition of alternating polymer layers over sacrificial cores that are removed at a later stage (82). Although LbL has been traditionally driven by electrostatic and hydrogen-bonding interactions, covalently bound films offer the advantage of a higher stability due to the formation of cross-linked polymer networks (82). For example, the group of Caruso has relied on a CuAAC-based LbL for

\section{Click Chemistry for Drug Delivery Nanosystems}


the preparation of $\mathrm{pH}-$ responsive polymer microcapsules by the assembly of ultrathin PAA films onto poly(ethylene imine) (PEI)-coated silica particles (83) (Fig. 19). In their approach, PAA copolymers incorporating $c a .10 \%$ of either alkyne or azide functionalities were synthesized by living radical polymerization. LbL assembly was performed by sequentially exposing PEI-coated particles to PAA-N 3 and PAA-Alk solutions in the presence of $\mathrm{CuSO}_{4}$ and sodium ascorbate. After centrifugation, the sacrificial silica cores were removed with fluoride to afford singlecomponent capsules with wall thickness of $5 \mathrm{~nm}$. One of the advantages of the present approach relates to the presence of remaining azide or alkyne groups on the shell of the capsules amenable to further selective functionalization. Following the same methodology, biodegradable capsules were prepared from homopoly(aminoacids), PLL or poly-L-glutamic acid (PGA), modified with azides and alkynes $\left(\mathrm{CuSO}_{4}\right.$, sodium ascorbate) (84). These capsules showed a reversible pHresponsive swelling/shrinking behavior, with potential applications for the $\mathrm{pH}$-triggered loading/release of bioactive species. In addition, PLL capsules were further PEGylated with NHS-PEG-OMe and NHS-PEG-biotin and their interaction with fluorescently labeled BSA and streptavidin investigated by flow cytometry. It was found that the non-specific BSA adsorption typical of PLL surfaces was considerably reduced in the PEGylated system, whereas streptavidin specifically bound to biotin. The biodegradability, low-fouling properties, and targeting ability of these capsules make them promising carries for targeted drug delivery.

\section{Fig. 19}

In a similar way, De Geest and coworkers have prepared biodegradable multilayer films and hollow capsules based on dextran (85). $\mathrm{CaCO}_{3}$ microparticles precoated with a layer of PAA-Alk were assembled by LbL with consecutive layers of azido- and alkyne-dextran under CuAAC conditions $\left(\mathrm{CuSO}_{4}\right.$, sodium ascorbate). Then, hollow capsules were obtained by

\section{Click Chemistry for Drug Delivery Nanosystems}


dissolving the $\mathrm{CaCO}_{3}$ core with EDTA at $\mathrm{pH}$ 5.2. The use of carbonate linkages for the incorporation of the azide and alkyne functionalities along the dextran chain ensured the biodegradability of the resulting capsules. The same authors have also prepared biodegradable microcapsules from azido- and alkyne-dextran by a microemulsion method using an external aqueous PEG phase $\left(\mathrm{CuSO}_{4}\right.$, sodium ascorbate) (86). When a FITC-labeled dextran was employed, the degradability of the capsules was studied under physiological conditions. It was demonstrated that the release of the entrapped FITC-dextran occurred in a controlled fashion, and the rate of release could be modulated by the density of cross-linking.

Caruso and coworkers have also developed a TEC-based LbL strategy that avoids the use of coupling reagents or catalysts (87) (Fig. 20). In this approach, a LbL assembly based on hydrogen-bonding is preformed on silica particles by depositing consecutive layers of PMAA (carrying thiol or alkene functionalities) and poly( $N$-vinylpyrrolidone) (PVP), that are subsequently cross-linked by irradiation with UV light (256 nm, acetate buffer). The efficiency of this TEC/LbL strategy was demonstrated by the lack of disassembly at $\mathrm{pH} 7$ under conditions leading to hydrogen-bonding disruption. The authors took advantage of the presence of remaining thiol or alkene functional groups on the surface of the capsules for the incorporation of PEG chains. A related CuAAC strategy has been more recently employed by the same group to prepare low-fouling polymer capsules decorated with antibodies showing specific binding to colorectal cancer cells (88). In this case, single component PVP capsules were prepared from the consecutive assembly of PVP-Alk and PMAA layers via hydrogen-bonding interactions, and further $\mathrm{CuAAC}\left(\mathrm{CuSO}_{4}\right.$, sodium ascorbate) cross-linking of the PVP layers with a bifunctional azide linker. After removal of the core template and PMAA layers, the remaining alkyne groups on the surface of the PVP capsules were conjugated to an azido-PEGylated antibody via CuAAC

\section{Click Chemistry for Drug Delivery Nanosystems}


( $\mathrm{CuSO}_{4}$, sodium ascorbate) in the presence of a $\mathrm{Cu}(\mathrm{I})$-chelating ligand [tris(4-carboxylbenzyltriazolylmethyl)amine]. Taking advantage of the same hydrogen-bonding assembly/CuAAC cross-linking sequential approach, Caruso and coworkers have recently prepared biodegradable capsules with drug-loaded multilayers as promising carrier systems for biomedical applications (89). For the preparation of these capsules, layers of PGA-Alk with/without covalently conjugated DOX molecules were assembled via hydrogen-bonding with PVP on silica templates, and then covalently stabilized using a diazide linker $\left(\mathrm{CuSO}_{4}\right.$, sodium ascorbate). After removal of PVP and the sacrificial template, DOX-loaded PGA capsules were obtained with control over drug dose and position in the multilayer system. These PGA capsules could be enzymatically degraded to release the active drug, which was localized in the cells nuclei.

\section{Fig. 20}

Although several methods for the preparation of polymeric capsules have been reported so far, all of them have in common the necessity of a pre-organized structure or template. A rare example of self-assembly into nanosized capsules not requiring the use of templates has been recently reported by Sung, Kim, and coworkers (90) . These authors made use of TEC (UV light, $\mathrm{MeOH})$ between dithiols and a cucurbit[6]uril carrying 12 allyloxy groups at the periphery for the preparation of polymer nanocapsules with high stability and relatively narrow size distribution (Fig. 21). Different dyes (carboxyfluorescein, rhodamine) have been entrapped inside these capsules. A unique property of these capsules is that they can form highly stable host-guest complexes with polyamines by virtue of the unique recognition properties of the accessible molecular cavities exposed on the capsule periphery.

\section{Fig. 21}

\section{Click Chemistry for Drug Delivery Nanosystems}


Several strategies taking advantage of click reactions have been also used for the surface functionalization of polymeric microspheres. For example, Chen and coworkers used CuAAC for the grafting of proteins onto biodegradable microspheres prepared from a P(LA-cocarbonate) carrying pendant alkyne groups (55). With this purpose, azido-decorated BSA was selected as a model protein. The effectiveness of the $\mathrm{CuAAC}$ conjugation $\left(\mathrm{CuSO}_{4}, \mathrm{Cu}\right.$ wire $)$ was demonstrated by confocal microscopy and X-ray photoelectron spectroscopy. In addition, CuAAC and TEC chemistries have been employed for the surface functionalization of microspheres with polymers as a strategy for enhancing their stability and modifying their properties $(91,92)$. For instance, the groups of Barner-Kowollik, Barner, and Müller have recently reported the surface modification of poly(divinylbenzene) microspheres with PNIPAM and PHEMA using either TEC (AIBN, acetonitrile) or $\mathrm{CuAAC}\left(\mathrm{CuSO}_{4}\right.$, sodium ascorbate, DMF- $\left.\mathrm{H}_{2} \mathrm{O}\right)$ strategies (92).

\section{METAL AND SILICA NANOPARTICLES}

During the last decades, hard-matter nanosystems, e.g., AuNP and other noble metal NP, MNP, QD, and SiNP, have been extensively investigated because of their unique physical and chemical properties, which strongly depend on their size and shape. Although these nanosystems are of great interest for researchers in different disciplines, they have been the focus of special attention in the biomedical field where they have found application as drug carriers, labeling agents, vectors for gene therapy, hyperthermia treatments, and magnetic resonance imaging (MRI) (93),(94). In addition, some of these nanostructures offer the possibility of simultaneously imaging and treating tumors (theranostic carriers), which may result advantageous over conventional chemotherapies.

\section{Click Chemistry for Drug Delivery Nanosystems}




\section{Gold and Noble-Metal Nanoparticles.}

AuNP and other noble-metal NP have emerged as novel nanostructures with exciting applications in therapy and diagnosis (e.g, drug and gene delivery, imaging, phototherapy, and radiotherapy enhancement treatments). Their low inherent toxicity, high surface area, and tunable stability have revealed noble-metal NP as promising platforms for biomedical applications (95),(94). Indeed, metallic NP have demonstrated their capability to deliver small drugs and large biomolecules such as peptides, proteins, or nucleic acids. Although monodisperse metal NP can be easily prepared with extremely small sizes $(<30 \mathrm{~nm})$, they usually suffer from a reduced chemical stability in solution and a high tendency to aggregate, which may lead to the loss of their unique properties. To overcome this shortcoming, the surface of the particles is usually functionalized with various types of ligands (e.g., surfactants, polymers, dendrimers, biomolecules), which in the case of AuNP typically involves thiolated species. In addition to the enhanced stability, the surface functionalization of the particles has been shown to be a key parameter for optimizing their bioavailability and non-immunogenicity. As a result, a great amount of work has been devoted to the effective functionalization of metal NP. Among different strategies used for this goal, those based on click chemistries have revealed extremely efficient.

The first example on the use of an AAC coupling for the surface decoration of AuNP was reported by Williams and coworkers (96). Azido-functionalized AuNP were decorated with various low molecular weight compounds bearing carbonyl groups adjacent to a terminal alkyne for improved reactivity. Although functionalization proceeded in the absence of catalyst, the use of organic solvents (dioxane or 1:1 hexane/dioxane) and the inability to achieve complete

\section{Click Chemistry for Drug Delivery Nanosystems}


functionalization of the terminal azides render this protocol of low applicability within the biomedical field. Subsequent attempts to improve reactivity by the use of organosoluble $\mathrm{Cu}$ catalysts led to particle aggregation or decomposition (96). These shortcomings have been partially solved by Astruc and coworkers who described experimental conditions for quantitative CuAAC functionalization of azide decorated AuNP [stoichiometric amounts of $\mathrm{CuSO}_{4}$ and sodium ascorbate, room temperature, THF- $\mathrm{H}_{2} \mathrm{O}$ (1:1), $\mathrm{N}_{2}$ atmosphere] (97). This optimized protocol has proven successful for the incorporation of organic, organometallic, dendritic, and short PEG chains on AuNP, but the requirement of large amounts of THF makes it unsuitable for the conjugation of proteins or nucleic acids.

Rowan, Brust, and coworkers used CuAAC to efficiently prepare functional enzyme-AuNP conjugates from an acetylene-functionalized Thermomyces lanuginosus lipase and azidodecorated water-soluble AuNP ( $\mathrm{CuSO}_{4}$, ascorbic acid) (98) (Fig. 22). The successful formation of the protein-particle conjugate was confirmed by gel electrophoresis, while a fluorometric lipase activity assay showed that approximately 7 fully active lipase molecules per particle had been coupled. Following a similar approach, the group of Rao has recently reported the sitespecific conjugation of bioluminescent Renilla luciferase proteins to AuNP for sensing protease activity (99). In addition, the conjugation of azido-functionalized AuNP to an alkyne-modified DNA duplex has been reported by the group of Simon $(\mathrm{CuBr}$, TBTA), leading to a chain-like assembly of NP on a DNA template (100). More recently, Pang and coworkers described a new method for the preparation of azido-bearing AuNP that were functionalized with Alk-HRP via $\mathrm{CuAAC}\left(\mathrm{CuSO}_{4}\right.$, ascorbic acid) rendering AuNP-HRP conjugates with retained catalytic activity $(101)$.

Fig. 22

\section{Click Chemistry for Drug Delivery Nanosystems}


$\mathrm{CuAAC}$ has also been effectively employed for the surface functionalization of silver (AgNP) and platinum (PtNP) nanoparticles. In various recent examples, Li and coworkers have reported the preparation of highly selective colorimetric sensors for $\mathrm{Cd}(\mathrm{II})$ and $\mathrm{Co}(\mathrm{II})$ that are based on triazole-ester (102) and triazole-carboxyl (103) modified AgNP prepared by CuAAC $\left(\mathrm{CuSO}_{4}\right.$, sodium ascorbate). Similarly, Carrot and coworkers have taken advantage of CuAAC (CuBr, PMDETA, dimethylacetamide) to graft biocompatible coronas of PEG and PCL onto azido-functionalized PtNP as hybrid structures with potential applications in biodetection (104).

Other click reactions that have found application for the surface functionalization of noblemetal NP include Michael addition and Diels-Alder. For instance, the group of Chechik has prepared shell cross-linked AuNP with improved stability via Michael addition of various aminodendrimers [poly(amido amine) and diaminobutane] to acrylate-terminated particles (105). Also, a reversible surface functionalization of AuNP has been accomplished by the group of Beyer by means of a Diels-Alder coupling strategy (106). AuNP were first functionalized with a thiolterminated PS- $b$-PEG prepared by Diels-Alder of the PS and PEG blocks. Subsequent thermal treatment of these particles caused retro Diels-Alder leading to AuNP functionalized by PS chains.

\section{Magnetic Nanoparticles}

In the last decade, MNP based on maghemite $\left(\gamma-\mathrm{Fe}_{2} \mathrm{O}_{3}\right)$ and magnetite $\left(\mathrm{Fe}_{3} \mathrm{O}_{4}\right)$ have consolidated as promising materials in biomedicine due to their applications mainly in drug delivery (93), MRI, and hyperthermia therapy (107). For these applications, particles must display high magnetic saturation, biocompatibility, and a functionalized surface. Although iron oxide NP with low dispersity are easily produced, they present an intrinsic instability, which is

\section{Click Chemistry for Drug Delivery Nanosystems}


normally overcome by installing protecting shells. This coverage not only protects MNP from degradation, but also provides anchoring sites for functionalization with active molecules or reactive groups.

The group of Turro has reported CuAAC conditions for the surface functionalization of $\gamma$ $\mathrm{Fe}_{2} \mathrm{O}_{3} \mathrm{NP}$ with low molecular weight organic species and polymers $[e . g ., \alpha$-acetylene-poly $($ tertbutyl acrylate)] (108). With this aim, short ligands containing carboxylic or phosphonic acid groups were designed as strong anchors to bind the NP surface. The presence of either a terminal azide or alkyne group in these ligands provided orthogonal functionality on the surface for further chemical modification by $\mathrm{CuAAC}\left(\mathrm{CuSO}_{4}\right.$, sodium ascorbate, $\left.\mathrm{DMSO}-\mathrm{H}_{2} \mathrm{O}\right)$.

A more biocompatible CuAAC protocol avoiding the need of organic solvents for the effective functionalization of MNP (silica oxide-coated $\mathrm{Fe}_{3} \mathrm{O}_{4}$ ) with biologically relevant ligands has been described by Lin and coworkers (109). Under the reported conditions $\left[\mathrm{CuSO}_{4}\right.$, tris(carboxyethyl)phosphine (TCEP), TBTA, pH 8], mannose, biotin, the FLAG peptide, the Tn antigen, and various proteins [EGFP and maltose binding protein (MBP)] were incorporated on the MNP surface and detected using fluorescently labeled antibodies/proteins. When MNP containing azides or alkynes at the surface were reacted with complementary alkyne- or azidoterminated mannose, the alkynated mannose was found to be more efficiently immobilized. This faster kinetic of the alkyne counterpart in solution is consistent with the required $\mathrm{Cu}(\mathrm{I})$ coordination of the alkyne. A similar preference has been reported when labeling CPMV (16),(23) and microarray slides (110). Interestingly, the activity of a MBP covalently immobilized on the MNP at its $\mathrm{C}$ terminus by site-specific CuAAC resulted higher than that by random amide bond formation, in agreement with the $\mathrm{C}$ terminus being distant from the MBP binding site.

\section{Click Chemistry for Drug Delivery Nanosystems}


Prosperi and coworkers have reported an interesting one-pot biofunctionalization of MNP $\left(\gamma-\mathrm{Fe}_{2} \mathrm{O}_{3}\right)$ via diazo transfer followed by in situ $\mathrm{CuAAC}$ (111). Since a common way to modify iron oxide NP involves their treatment with $\gamma$-aminopropyl triethoxysilane to give aminofunctionalized MNP, these authors explored the possibility of generating azido functionalities from the corresponding amines via $\mathrm{Cu}(\mathrm{II})$-catalyzed diazo transfer (112), and their subsequent funtionalization via $\mathrm{CuAAC}$. In this one-pot protocol, the $\mathrm{Cu}(\mathrm{I})$ species required for the $\mathrm{CuAAC}$ are easily generated in situ by addition of sodium ascorbate to the $\mathrm{Cu}(\mathrm{II})$ salts necessary for the diazo transfer (biphasic $\mathrm{CH}_{2} \mathrm{Cl}_{2}-\mathrm{H}_{2} \mathrm{O}$ mixture). By application of this procedure, the biofunctionalization of MNP with lactose and HSA has proceeded successfully and with retained biological activity.

In a recent report, Mirkin and coworkers employed $\mathrm{CuAAC}\left(\mathrm{CuSO}_{4}\right.$, sodium ascorbate, THPTA, DMSO- $\mathrm{H}_{2} \mathrm{O}$ ) to conjugate alkynated oligonucleotides to superparamagnetic iron oxide nanoparticles (SPION) functionalized with azides (113). The resulting densily functionalized SPION exhibited good cellular uptake by HeLa cells without the need of transfection agents. Another example of SPION bioconjugation was reported by Tsourkas and coworkers (114). The authors combined expressed protein ligation (EPL) with CuAAC to produce a highly efficient and site-specific conjugation strategy that was employed in the preparation of SPION labeled with HER2/neu-targeted affibodies. In their approach, the affibodies expressed in bacteria were first conjugated to an alkynated fluorescent peptide via EPL. CuAAC was then used for the conjugation to azido-labeled SPION ( $\mathrm{CuSO}_{4}$, sodium ascorbate, bathocuproinedisulfonic acid). The resulting HER2-SPION proved to be highly potent and receptor-specific both in vitro cell studies and murine tumor models. In addition, the authors demonstrated the application of this EPL-CuAAC strategy to other nanostructures, such as liposomes and dendrimers.

\section{Click Chemistry for Drug Delivery Nanosystems}


MNP are also useful in magnetic hyperthermia, a promising cancer thermotherapy based on the exothermic properties of magnetic materials under the influence of an alternating current magnetic field (107). In order to specifically target certain tumors, MNP can be conjugated with tumor-specific targeting ligands, thereby minimizing undesired side effects. In a recent report, Yogo and coworkers prepared FA-functionalized $\mathrm{Fe}_{3} \mathrm{O}_{4} \mathrm{NP}$ by $\mathrm{CuAAC}$ as a cancer targeting system with application in hyperthermia treatment (115). Taking into account the low chemoselectivity in the derivatization of FA through the carboxylic acids, and the relevance of the $\alpha$-carboxyl group for high-affinity binding to the FR, these authors developed a novel alkynation method at the heterocycle moiety by treatment with 2,3-dibromopropionyl chloride followed by $\mathrm{HBr}$ elimination. The resulting Alk-FA was subsequently reacted with azidoterminated MNP under standard CuAAC conditions (CuI, DMSO- $\left.\mathrm{H}_{2} \mathrm{O}\right)$. In a further step, Yogo and coworkers prepared SPION functionalized with FA and CD as novel devices for simultaneous drug delivery and hyperthermia treatment (116). With this aim, $\beta$-CD was used as nanocontainer for the anticancer drug tamoxifen and as anchoring point for the covalent CuAAC $\left(\mathrm{CuI}, 70{ }^{\circ} \mathrm{C}\right)$ functionalization of the SPION with FA. Controlled release of tamoxifen was achieved by applying a high frequency magnetic field at an optimum temperature for hyperthermia, demonstrating that induction heat can be useful not only for hyperthermia treatment, but also as a driving force for drug release.

Perez and coworkers have also relied on $\mathrm{CuAAC}$ for the incorporation of a FA-N $\mathrm{N}_{3}$ derivative at the PAA-Alk coating of iron oxide NP (CuI, DMF- $\left.{ }_{2} \mathrm{O}\right)$ (117) (Fig. 23). In addition, taxol and a near-infrared dialkylcarbocyanine dye were encapsulated within hydrophobic pockets at the polymeric matrix, resulting in a multifunctional (imaging and therapy) and multimodal (magnetic and fluorescent) folate-derivatized NP. This system provided targeted drug delivery to lung

\section{Click Chemistry for Drug Delivery Nanosystems}


carcinoma A549 cells that overexpress the FR, without affecting normal cells not overexpressing this receptor. Similarly, Weissleder and coworkers prepared dextran-coated SPION that were functionalized with the radionuclide ${ }^{18} \mathrm{~F}$ in high yield via $\mathrm{CuAAC}\left(\mathrm{CuSO}_{4}\right.$, sodium ascorbate, BPDS, DMSO- $\mathrm{H}_{2} \mathrm{O}$ ) for in vivo PET (position emission tomography) imaging (118),(119).

\section{Fig. 23}

Bhatia and coworkers have investigated the in vivo targeting of iron oxide NP to tumors (120). $\mathrm{CuAAC}\left(\mathrm{CuSO}_{4}\right.$, sodium ascorbate) was selected to specifically conjugate the highly functionalized cyclic tumor targeting peptide LyP-1 to azido-functionalized PEGylated magnetofluorescent NP. LyP-1 has been reported to bind p32, a mitochondrial protein that is both overexpressed and aberrantly localized at the surface of tumor cells, macrophages, and lymphatic endothelial cells in certain experimental tumors and in human cancers. The resulting NP were able to direct their binding to p32-expressing tumor cells in vitro. More importantly, these NP have been shown to be stable for more than $5 \mathrm{~h}$ in the blood stream after intravenous administration, allowing them to extravasate into tumors, penetrate the tumor interstitium, and specifically bind to p32-expressing cells. Altogether, these findings not only support the excellent features of CuAAC in bioconjugation (orthogonality, efficiency, aqueous compatibility), but confirms the stability of the clicked complexes in vivo in the blood and tumor environments.

Although thiol-ene chemistry has been widely employed in the field of polymeric colloidal systems, its use for the modification of MNP is still limited, with only a few recent examples being reported. One of the main shortcomings in the application of this chemistry to MNP is the difficulty in synthesizing alkene-immobilized particles because C-C double bonds undergo polymerization at the high temperature required in this process. This limitation has been recently

\section{Click Chemistry for Drug Delivery Nanosystems}


overcome by Yogo and coworkers by employing allyl groups, which do not undergo polymerization (121). The authors reported the modification of allyl-functionalized $\mathrm{Fe}_{3} \mathrm{O}_{4} \mathrm{NP}$ with cysteine via a one-pot approach hydrolysis-condensation of $\mathrm{Fe}(\mathrm{III})$ allylacetylacetonate and TEC (AIBN, EtOH- $\mathrm{H}_{2} \mathrm{O}, 60{ }^{\circ} \mathrm{C}$ ). A different strategy was employed by Warner and coworkers, who employed $\mathrm{Fe}_{3} \mathrm{O}_{4}$-mercaptopropionic acid NP that were further decorated with allyl diphosphonic acid or ester ligands by TEC (benzophenone, UV light, $\mathrm{H}_{2} \mathrm{O}$ or $\mathrm{MeOH}$ ) (122).

\section{Quantum Dots}

In a similar fashion to noble-metal NP, the inorganic semiconductor nanocrystals known as QD have drawn attention in the fields of diagnosis and imaging due to their unique fluorescence properties, such as broad absorption, narrow and symmetric emission spectra, long luminescence lifetime, and good photostability (123). These optical properties can be tuned as a function of size and surface functionality. In spite of these favorable properties, the development of efficient and mild bioconjugation strategies for the surface functionalization of QD with biologically relevant molecules remains an important challenge to be addressed. Unfortunately, some of the currently most popular click reactions have been shown as having severe limitations in their application for this goal. For instance, as thiols are commonly employed as stabilizing ligands in the preparation of $\mathrm{QD}$, their use for surface functionalization via TEC or Michael additions is severely restricted since ligand exchange at the surface of the QD may occur. On the other hand, the application of CuAAC suffers from two main drawbacks: (i) The harsh conditions usually employed for the preparation of QD are incompatible with the presence of azide or alkyne groups in the stabilizing ligands. Accordingly, ligand exchange strategies have to be employed for the incorporation of these functional groups, which usually result in reduced

\section{Click Chemistry for Drug Delivery Nanosystems}


photoluminescence quantum yields (PTQY); (ii) $\mathrm{Cu}$ catalysts promote a strong inhibition of the QD luminescence. These shortcomings were early illustrated by the group of Binder in the functionalization of CdSe QD by CuAAC (124). A ligand exchange method was employed to decorate trioctylphosphine oxide QD with azide or alkyne functional groups. This process resulted in a reduction of PTQY of up to $63 \%$. In addition, CuAAC derivatization of the resulting alkyne-functionalized conjugate (CuBr, DIPEA, TBTA, THF) led to a dramatic decrease of the PTQY, so that thermal AAC conditions had to be implemented.

An effective way to overcome the above mentioned drawbacks in QD functionalization is the application of convenient $\mathrm{Cu}$-free cycloadditions. In this regard, Texier and coworkers have recently described the application of SPAAC for the functionalization of PEG-decorated $\mathrm{CdSe} / \mathrm{ZnS} \mathrm{QD}$ and their application to the in vitro imaging of cell membrane glycoproteins (125). A cyclooctyne-functionalized QD was prepared from a commercially available $\mathrm{CdSe} / \mathrm{ZnS} / \mathrm{PEG}-\mathrm{NH}_{2} \mathrm{QD}$, and then functionalized with an azido-tagged mannosamine in the presence and absence of $\mathrm{Cu}$ (Fig. 24). In this way, while the SPAAC decoration had a minor effect on the PTQY (7\% as compared to the cyclooctyne-functionalized QD), the addition of $\mathrm{Cu}$ led to a marked decrease of more than $65 \%$.

\section{Fig. 24}

In another approach, Bewendi and coworkers have relied on the inverse-electron-demand Diels-Alder cycloaddition between tetrazine and strained alkenes (norbornene) to functionalize CdSe QD with both Alexa 594 and an epidermal growth factor (EGF) (126). A ligand exchange protocol was employed for the surface doping of QD with multiple norbornene units which resulted in a reduction of $40 \%$ in the PTQY. No additional quenching of fluorescence occurred during Diels-Alder cycloaddition. Interestingly, the EGF-QD proved to be efficient for the in

\section{Click Chemistry for Drug Delivery Nanosystems}


vitro visualization of A431 human carcinoma cells via an in situ labeling approach or with preformed conjugates (Fig. 25).

\section{Fig. 25}

\section{Silica Nanoparticles}

SiNP represent another class of nanomaterials with potential applications in drug delivery, biodetection, and diagnosis, that possess an excellent biocompatibility, virtually no toxicity, and reactive functional groups on the surface available for functionalization (127). Moreover, a variety of functional groups can be attached to the silica surface via silane linkers. Following this principle, the group of Brittain has reported a combination of RAFT polymerization and CuAAC to graft polymers onto SiNP $(128,129)$. These authors have used an unprotected alkyneterminated RAFT CTA to prepare alkyne-terminated polymers and block copolymers, i.e., PS, polyacrylamide, PMA, and PS- $b$-PMA, which were subsequently grafted onto azidofunctionalized $\mathrm{SiNP}$ with a high density of grafting $\left(\mathrm{CuSO}_{4}\right.$, sodium ascorbate, $\mathrm{DMSO}-\mathrm{H}_{2} \mathrm{O}$ or DMF- $\mathrm{H}_{2} \mathrm{O}$ ). Using the same CTA, these authors have also reported a tandem RAFT polymerization/CuAAC procedure to modify the surface of SiNP in a controlled manner.

Janus-type SiNP decorated with biomolecules on one hemisphere and biocompatible polymer brushes on the other have huge potential applications in targeted drug delivery, as well as in the development of switchable devices, optical probes and emulsion stabilizers. Liu, Zhao, and coworkers have reported on the use of CuAAC for the sequential functionalization of azidemodified SiNP embedded on the surface of PS particles used as scaffolds (130) (Fig. 26). In this approach, the originally exposed hemisphere of the SiNP was coupled to an alkynated biotin derivative by means of $\mathrm{CuAAC}\left(\mathrm{CuSO}_{4}\right.$, sodium ascorbate, $\left.t-\mathrm{BuOH}-\mathrm{H}_{2} \mathrm{O}\right)$. After removal of the

\section{Click Chemistry for Drug Delivery Nanosystems}


excess of biotin and catalyst by extensive dialysis, the product was collected by centrifugation. Then, the PS was removed by dispersion in THF followed by centrifugation, yielding SiNP with biotin groups on one hemisphere and azide groups on the other. The bioactivity of the conjugated biotin molecules was confirmed by an avidin/HABA competitive binding assay. The remaining azide groups on the surface were coupled to an alkynated PEG through a second CuAAC $\left(\mathrm{CuSO}_{4}\right.$, sodium ascorbate, $\left.\mathrm{H}_{2} \mathrm{O}\right)$, leading to Janus-type SiNP differently functionalized with biotin and PEG at both hemispheres.

Fig. 26

Müller and coworkers have reported a new type of hybrid NP consisting of a fluorescent inorganic silica-like core and a biocompatible polymer shell containing terminal alkynes amenable to functionalization by CuAAC (131). With this aim, block copolymers composed of a poly[oligo(ethylene glycol)acrylate] hydrophilic block and a hydrophobic block containing trimethoxysilane-functionalized and dye-substituted monomers [(3-acryloxypropyl)trimethoxysilane and 1-pyrenebutyl acrylate] were prepared by RAFT polymerization. For the polymerization, an alkynated CTA was used to introduce a terminal alkyne group at the hydrophilic block. The selection of the trimethoxysilane monomer at the hydrophobic block was based on its ability to undergo cross-linking into a stable silsesquioxane network under basic conditions for increased stability. Additionally, pyrene containing monomers were included as fluorescent tags. After self-assembly of the block copolymers, the resulting micelles were core cross-linked by mild treatment with ammonia. The resulting alkynated particles were functionalized with Rhodamine $\mathrm{B}$ by means of $\mathrm{CuAAC}$ under drastic conditions $(\mathrm{CuBr}$, bipy, 80 ${ }^{\circ} \mathrm{C}$, degassed DMF). The potential of these stabilized fluorescent SiNP as biocompatible carriers for intracellular delivery was nicely demonstrated by in vitro experiments on lung cancer cells.

\section{Click Chemistry for Drug Delivery Nanosystems}


An interesting report on the dual, bioorthogonal labeling of SiNP has been recently described by Kele, Wolfbeis, and coworkers by sequentially exploiting SPAAC and CuAAC reactions $(132,133)$ (Fig. 27). With this aim, SiNP were doped with several copies of a coumarin dye and azide reporter groups. The resulting fluorescent NP were then conjugated via SPAAC with a synthetic dipeptide containing both a cyclooctyne and a terminal alkyne. Subsequent CuAAC labeling of the alkynated peptides with a specifically designed azido-coumarin fluorescent dye yielded dually labeled NP as efficient FRET systems. The same authors have adapted this strategy for the preparation of diagnostic NP carrying a heptapeptide for detection of MMP-2, an enzyme of the collagenase family that plays a key role in several physiological processes, including cancer progression. The successful application of the same FRET pair for the labeling of proteins (BSA) paves the way for the use of this methodology in the construction of dually labeled conjugates for alternative biomedical purposes.

\section{Fig. 27}

In a recent example, the group of Bein has reported the CuAAC funtionalization of coreshell colloidal mesoporous SiNP for the preparation of stimuli-responsive porous materials for the controlled release of guest molecules (134). Colloidal mesoposous SiNP with azide groups at the outer shell were prepared and functionalized by $\mathrm{CuAAC}\left(\mathrm{CuSO}_{4}\right.$, sodium ascorbate, THPTA) with double stranded oligodeoxyribonucleotides incorporating an azide and a biotin label at different strands. As proof of concept, fluorescein selected as model compound was encapsulated, and then the pores closed by complexation to avidin. These functionalized SiNP showed a programmable thermoresponsive behavior that allowed the controlled release of the guest molecules after melting the DNA molecular valves, a characteristic that is encoded by the length of the oligonucleotide employed.

\section{Click Chemistry for Drug Delivery Nanosystems}




\section{CARBON NANOTUBES AND FULLERENES}

CNT and fullerenes are nanostructures composed solely of carbon atoms that have been thoroughly studied in a wide variety of disciplines due to their unique physical and chemical properties (135). Fullerenes are highly symmetric cage molecules, of which $\mathrm{C}_{60}$ (Buckminsterfullerene) is the foremost member: a truncated icosahedron with a diameter of $0.7 \mathrm{~nm}$, containing 60 carbon atoms forming 20 hexagons and 12 pentagons. Similarly, CNT are composed of carbon atoms arranged in benzene rings, forming graphene sheets rolled up to give cylinders with typical diameters in the range 1-100 $\mathrm{nm}$. Two main types of CNT exist, single-walled (SWCNT) and multi-walled carbon nanotubes (MWCNT), the latter being formed by several concentric layers of rolled graphene. One of the main shortcomings for the development of biomedical applications of fullerenes and CNT is their poor solubility in aqueous media. As a result, great efforts have been dedicated to increase their solubility in water and biocompatibility by different strategies, including the chemical modification by click chemistry (136),(137).

For instance, Adronov and coworkers have reported conditions for the surface functionalization of SWCNT by means of CuAAC (138). With this aim, p-aminophenyl propargyl ether was reacted with SWCNT using a solvent-free diazotization/coupling procedure leading to alkyne-decorated SWCNT with a high degree of functionalization. Conditions for the conjugation of this SWCNT with an azido-terminated PS (prepared by ATRP and subsequent bromine end-group substitution with $\mathrm{NaN}_{3}$ ) involved $\mathrm{CuI}$ and DBU in DMF. In an attempt to improve the biocompatibility and water solubility of SWCNT, Zheng and coworkers have exploited this strategy for the covalent linkage of azido-functionalized $\beta$-CD (CuI, DBU, DMF,

\section{Click Chemistry for Drug Delivery Nanosystems}


$\left.70{ }^{\circ} \mathrm{C}\right)(139)$ (Fig. 28). The inclusion complexation behavior of this artificial receptor with quinine was studied in aqueous solution by fluorescence spectroscopy. The higher biocompatibility and rich guest recognition properties derived from the $\mathrm{CD}$ make this $\mathrm{CD}$ SWCNT hybrid a promising platform for materials science and drug delivery applications.

\section{Fig. 28}

An alternative versatile approach to modify the surfaces of CNT has been described by the group of Gao by a combination of CuAAC "grafting onto" and ATRP "grafting from" strategies (140). A clickable macroinitiator $\mathrm{PBrN}_{3} \mathrm{PrMA}$, carrying bromides for ATRP as well as azido groups for CuAAC, was synthesized and coupled to alkynated SWCNT/MWCNT by CuAAC (CuBr, PMDETA, DMF). Poly(n-butylmethacrylate), PS, and PEG were subsequently grafted onto/from the resulting CNT via ATRP/CuAAC to afford CNT-supported amphiphilic polymer brushes with controlled degree of grafting.

Because of their unique electronic properties, the applications of functionalized CNT are mainly focused on the fields of solar energy conversion, electronics, and sensing. In this regard, several groups have reported the application of $\mathrm{CuAAC}$ for the functionalization of $\mathrm{CNT}$ with AuNP (141), MNP (142), and porphyrin dendrons (143), as potential platforms for novel electronic materials. In addition, CuAAC has been applied for the preparation of sugar-based amphiphiles capable of binding CNT surfaces through $\pi-\pi$ interaction and function as homogeneous bioactive coatings to reduce cytotoxicity and facilitate targeting (144). The combination of these principles (good electronic properties, reduced toxicity and active targeting) points out potential applications of CNT in drug delivery, where click chemistry will play a critical role as coupling technology.

\section{Click Chemistry for Drug Delivery Nanosystems}


Functionalized fullerenes, especially those based on the $\mathrm{C}_{60}$ platform, have been recently investigated as novel materials in nanomedicine. Thus, $\mathrm{C}_{60}$ has been used for the development of HIV-1 protease inhibitors, drug and gene delivery systems, or novel photodynamic therapy and MRI contrast agents (137).

The application of CuAAC for the construction of functionalized fullerenes has allowed the preparation of complex conjugates where previous synthetic approaches failed. In one example by Isobe, Nakamura, and coworkers, C5-symmetric multivalent glycoconjugates with suitable spatial orientation for the inhibition of pentameric Shiga-like toxin proteins have been prepared from a pentaalkynyl $\mathrm{C}_{60}$ derivative and various unprotected azido-functionalized carbohydrates, including the $\mathrm{P}^{\mathrm{k}}$ trisaccharide present in the natural ligand globotriaosylceramide (Gb-3) (CuBr·SMe2, $i$-Pr2EtN, DMSO, $\left.40{ }^{\circ} \mathrm{C}\right)(145)$ (Fig. 29).

\section{Fig. 29}

Shortly after, Nierengarten and coworkers prepared symmetrical $\mathrm{C}_{60}$ hexakis-dendritic adducts bearing 12 peripheral azide or alkyne groups, that were functionalized with various ligands, including ferrocene, a porphyrin, and G2 Fréchet-type dendrons $\left(\mathrm{CuSO}_{4}\right.$, sodium ascorbate, biphasic $\mathrm{CH}_{2} \mathrm{Cl}_{2}-\mathrm{H}_{2} \mathrm{O}$ mixture) (146),(147) (Fig. 29). This methodology also enabled the preparation of difunctionalized fullerenes via a sequential double $\mathrm{CuAAC}$ approach starting from a $\mathrm{C}_{60}$ hexaadduct bearing 10 azides and 2 TMS-protected alkyne groups (147). Biologically relevant ligands such as carbohydrates have been attached to the periphery of $\mathrm{C}_{60}$ using this methodology. Some of the resulting "sugar balls" have been shown to be efficient inhibitors of glycosidase activity, and in some cases, a positive multivalent effect was observed (148).

$\mathrm{CuAAC}$ has also allowed the preparation of polymer- $\mathrm{C}_{60}$ hybrids. In the first example, reported by the group of Cheng (149), a PS-C 60 conjugate was obtained by reacting azido-

\section{Click Chemistry for Drug Delivery Nanosystems}


terminated PS of different molecular weights with a monoalkynated fullerene derivative $(\mathrm{CuBr}$, PMDETA, toluene).

Steinmetz, Finn, Manchester, and coworkers have explored the advantages of combining fullerenes and viral nanoparticles (VNP) for biomedical applications, such as photodynamic tumor therapy (150). VNP were decorated with $\mathrm{C}_{60}$ using classical amide formation conditions and CuAAC. An Alk-PEG-C60 conjugate was prepared and reacted with an azide modified VNP $\left(\mathrm{CuSO}_{4}\right.$, sodium ascorbate, THPTA, aminoguanidine), resulting in soluble and stable hybrids with a significantly higher loading than via amides. The covalent incorporation of $\mathrm{C}_{60}$ was verified by Western blotting and STEM. The structural integrity of the VNP-PEG-C 60 hybrid was confirmed by SEC, TEM, STEM, and native gel electrophoresis. The cellular uptake of a dyelabeled VNP-PEG-C60 was studied in HeLa human cancer cells using confocal microscopy, which revealed that internalization was not inhibited by the $\mathrm{C}_{60}$ units.

The application of TEC to the functionalization of fullerenes has been limited by their reactivity towards thiyl radicals (151), which can lead to undesired byproducts. In spite of this, the group of Nierengarten has taken advantage of the higher reactivity of the methacrylate group to decorate the surface of $\mathrm{C}_{60}$ with model thiols (AIBN, benzene, $80^{\circ} \mathrm{C}$ ) (152). In addition, the authors exploited the orthogonality of TEC (AIBN, degassed THF, reflux) and CuAAC (CuSO 4 , sodium ascorbate, biphasic $\mathrm{CH}_{2} \mathrm{Cl}_{2}-\mathrm{H}_{2} \mathrm{O}$ mixture) to demonstrate the advantages of sequential click reactions to yield differently functionalized fullerenes with potential applications in the biomedical field (Fig. 30).

Fig. 30

\section{BIONANOPARTICLES}

\section{Click Chemistry for Drug Delivery Nanosystems}


BNP are supramolecular protein assemblies such as viruses, virus-like particles, ferritins, and enzyme complexes, receiving great attention as pre-fabricated, biocompatible, and biodegradable scaffolds for a wide variety of nanotechnology applications, including drug delivery (153). Since azides and alkynes are inert towards biological environment, CuAAC has revealed as an excellent tool for the functionalization of BNP. Fokin, Sharpless, Finn, and coworkers reported the first $\mathrm{CuAAC}$ functionalization of a BNP, the CPMV, a $31 \mathrm{~nm}$ structurally rigid icosahedral assembly of 60 identical copies of a two-protein asymmetric unit around a single-stranded RNA (16). With this purpose, the surface of CPMV was first decorated with 60 azide or alkyne groups at reactive lysine or cysteine residues in a controlled way. Adequate CuAAC experimental conditions involved the use of $\mathrm{CuSO}_{4}$, TBTA, and either TCEP or a $\mathrm{Cu}$ wire $(5 \% t$-BuOH in $\mathrm{PBS}, \mathrm{pH}$ 8). Under these conditions, quantitative functionalization of CPMV with 60 copies of a complementary azide- or alkyne-functionalized fluorescein resulted, without compromising the structural integrity of the viral particles.

In spite of the successful bioconjugation achieved, some limitations of CuAAC for the functionalization of biomacromolecules were pointed out by the authors in this study. Thus, in the absence of TBTA, viral capsides resulted either degraded or aggregated by $\mathrm{Cu}$ ions or the reducing agents used to generate $\mathrm{Cu}(\mathrm{I})$ in situ (16). As a result, great efforts have been devoted to minimize these shortcomings of CuAAC in bioconjugation. As previously described in this Expert Review, this goal has been generally achieved by the proper selection of a $\mathrm{Cu}(\mathrm{I})$-chelating ligand, intended to stabilize the oxidation state of $\mathrm{Cu}(\mathrm{I})$, increase the reaction rate, and sequester $\mathrm{Cu}$ ions to facilitate purification. TBTA is indeed a good example of $\mathrm{Cu}(\mathrm{I})$-chelating ligand. Its tetradentate binding ability allows the formation of $\mathrm{Cu}(\mathrm{I})$ chelates so stable, that exclusion of $\mathrm{O}_{2}$ from the reaction medium is not required in order to avoid the oxidation of $\mathrm{Cu}(\mathrm{I})$ (20). On the

\section{Click Chemistry for Drug Delivery Nanosystems}


other side, its poor solubility in water has encouraged the search of alternative ligands with increased solubility (i.e., BPDS and THPTA).

BPDS in the presence of $\mathrm{Cu}(\mathrm{I})$ salts $\left[\right.$ e.g., $\mathrm{Cu}(\mathrm{MeCN})_{4} \mathrm{OTf}, \mathrm{Cu}(\mathrm{MeCN})_{4} \mathrm{PF}_{6}, \mathrm{CuBr}$ ] represents an interesting water soluble alternative to TBTA. Finn and coworkers have reported the successful CuAAC functionalization of CPMV under these conditions with a plethora of biologically relevant substrates, such as complex carbohydrates (sialyl Lewis X, blood group A antigen, tri-LacNAc, globo-H, and a tetrasaccharide galectin-4 ligand), peptides (RGD and a portion of the protective antigen of anthrax toxin), polymers (PEG and a glycopolymer produced by ATRP), a DOTA-Gd complex, and the iron carrier protein transferrin [HEPES (usually better results than Tris or PBS buffers), $\mathrm{pH} 8$, rigorous exclusion of $\left.\mathrm{O}_{2}\right](23),(154,155,156)$ (Fig. 31). Interestingly, the high catalytic activity of BPDS renders unnecessary the use of a large excess of coupling probes. As previously observed with MNP (109) and microarray slides (110), CuAAC on CPMV containing peripheral azides proceeded more efficiently than with alkynes.

\section{Fig. 31}

The BPDS/Cu(I) system has been also exploited by Finn, Manchester, and coworkers for the functionalization of CPMV with FA (157). With the aim of overcoming the natural targeting ability of CPMV towards several mammalian cell lines and tissues in vivo, and redirecting it to alternative targets, FA was selected as a model ligand. FA was attached to CPMV by CuAAC through a short PEG linker, and the specific recognition by tumor cells bearing the FR studied. The incorporation of PEG-FA completely eliminated the background binding of CPMV, and allowed the specific recognition by tumor cells bearing the FR. The analysis different loadings of FA on CPMV revealed that high density loadings may not be necessary for efficient targeting to tumor cells. In a more recent contribution, the group of Finn has also relied on the BPDS/Cu(I)

\section{Click Chemistry for Drug Delivery Nanosystems}


system for the CuAAC functionalization of the capsid of bacteriophage $\mathrm{Q} \beta$ labeled with unnatural amino acids, containing azides or terminal alkynes introduced by site-directed mutagenesis (158).

The combination of BPDS and $\mathrm{Cu}(\mathrm{I})$ salts has been adopted by many other research groups as standard $\mathrm{CuAAC}$ conditions for the bioconjugation of BNP. For example, the group of Fisher and Manchester used these conditions for the functionalization of the rod-shaped potato virus X with fluorescent dyes (159). In another example, Wang and coworkers chemoselectively functionalized the surface of horse spleen apoferritin, a cage-like protein structure composed of 24 structurally equivalent subunits (160). A total of 4 reactive lysines per subunit (K83, K97, K104, and K143) were first functionalized with terminal alkynes and subsequently decorated with an azido-coumarin by $\mathrm{CuAAC}$. Interestingly, while initial $\mathrm{CuAAC}$ attempts with $\mathrm{CuSO}_{4} /$ ascorbic acid or $\mathrm{CuSO}_{4} / \mathrm{TCEP}$ led to aggregation or denaturation of the apoferritin, the use of $\mathrm{CuBr} / \mathrm{BPDS}$ afforded functionalized intact protein particles as confirmed by sizeexclusion FPLC and TEM. The incorporation of one coumarin per subunit was confirmed by MALDI-MS analyses of a V8 protease digests.

More recently, Carrico and coworkers have employed these CuAAC conditions $(\mathrm{CuBr} / \mathrm{BPDS})$ for the selective functionalization of human adenovirus type 5 (161). Effective decoration of the viral surface was accomplished via metabolic incorporation of $\mathrm{N}$ azidoacetylglucosamine into the fiber protein during virus production, and subsequent $\mathrm{CuAAC}$ conjugation with alkynated derivatives of the FLAG peptide, tetramethylrhodamine, and FA. Remarkably, a folate-decorated adenovirus encoding a GFP in the viral genome showed a 3-4fold increase GFP expression in FR-expressing mouse breast carcinoma cells (4T1) compared to the unmodified adenovirus.

\section{Click Chemistry for Drug Delivery Nanosystems}


Young, Douglas, and coworkers also reported a CuAAC strategy based on the use of BPDS and $\mathrm{Cu}(\mathrm{MeCN})_{4} \mathrm{OTf}$ for the synthesis of a cross-linked dendritic network in the interior of the porous heat shock protein cage (Hsp) (162). With this aim, a mutant HspG41C carrying a genetically introduced cysteine reactive site on the interior surface of each 24 identical subunits was selected. Dendritic structures were grown within the cage by reacting the cysteine residue with $\mathrm{N}$-propargyl bromoacetamide and subsequent iterative CuAAC couplings with 2-azido-1azidomethyl ethylamine and tripropargyl amine to generate an internal branched structure that finally cross-linked (Fig. 32). The resulting internally functionalized cages were monodisperse and very similar in size to the native protein cage (SEC, TEM, DLS). In addition, the crosslinked cages benefited from extended thermal stability to at least $120{ }^{\circ} \mathrm{C}$ and an enhanced internal carrying capacity with potential application in drug delivery.

\section{Fig. 32}

As seen above, the use of BPDS in connection with $\mathrm{Cu}(\mathrm{I})$ salts has allowed the efficient and clean functionalization of various types of BNP under conditions that avoid degradation and aggregation by $\mathrm{Cu}, \mathrm{ROS}$, and reducing agents. Nevertheless, the high sensitivity of this catalytic system to air oxidation (requiring rigorous exclusion of $\mathrm{O}_{2}$ or the use of a sacrificial reducing agent) demanded more user-friendly reaction conditions for the widespread application of CuAAC in bioconjugation. With this aim, the group of Finn has developed new experimental procedures, including an electrochemically triggered CuAAC (25) and the use of the water soluble ligand THPTA (22). In the first approach, $\mathrm{Cu}(\mathrm{II})$ is electrochemically reduced to $\mathrm{Cu}(\mathrm{I})$ in the presence of the coupling substrates and BPDS (Fig. 4). Under these conditions, no reducing agent is required and the $\mathrm{O}_{2}$ present in the reaction medium is reduced to $\mathrm{H}_{2} \mathrm{O}$ (i.e., $\mathrm{O}_{2}+4 \mathrm{H}^{+}+$ $4 \mathrm{e}^{-} \rightarrow 2 \mathrm{H}_{2} \mathrm{O}$ ). Consequently, degradation processes by ROS or reducing agents are minimized

\section{Click Chemistry for Drug Delivery Nanosystems}


(25). The versatility of this methodology was demonstrated by conjugating up to 650 copies of an alkynated selenomethionine to azido-modified bacteriophage $\mathrm{Q} \beta\left(\mathrm{CuSO}_{4}, \mathrm{BPDS}\right.$, electrolysis, $-200 \mathrm{mV}$ ) with comparable outcome to that previously reported under $\mathrm{O}_{2}$-free conditions (25) (Fig. 4). The second approach involves a protocol relying on the simplicity and reliability of the $\mathrm{Cu}(\mathrm{II}) /$ ascorbate system and the water solubility of THPTA (22). THPTA, originally reported by the group of Fokin (20), had been previously claimed by Brown and coworkers as an effective ligand for the harmless CuAAC functionalization of nucleic acids (26). In addition to the known $\mathrm{Cu}(\mathrm{I})$-chelating properties, Finn and coworkers have reported THPTA as radical scavenger, strongly accelerating the decomposition of $\mathrm{H}_{2} \mathrm{O}_{2}$ generated in the reaction medium [5-fold excess of THPTA $\left.v_{s} \mathrm{Cu}(\mathrm{I})\right]$. Under these conditions, the use of the carbonyl capturing reagent aminoguanidine (stoichiometric aminoguanidine/ascorbate ratio) is also recommended to minimize the adverse effects of ascorbate byproducts on amine-containing substrates. Following this protocol, functionalization of bacteriophage $\mathrm{Q} \beta$ with up to 630 copies of a fluorescein dye or 50 BSA molecules has been efficiently accomplished (Fig. 4).

The advantages of this CuAAC bioconjugation protocol [Cu(II)/ascorbate/THPTA] have been also demonstrated by the group of Douglas for the internal functionalization of the mutant protein cage HspG41C with a branched iron-phenanthroline-based coordination polymer (163). Also, by the group of Finn for the covalent functionalization of bacteriophage $\mathrm{Q} \beta$ with $\mathrm{C}_{60}$ Buckyballs (150), and the human iron-transfer protein transferrin, a high affinity ligand for receptors upregulated in a variety of cancer cells (164). The transferrin conjugation to $\mathrm{Q} \beta$ particles allowed specific recognition by transferrin receptors and cellular internalization through clathrin-mediated endocytosis, as determined by fluorescence microscopy on cells expressing GFP-labeled clathrin light chains. More recently, these conditions have been also applied by

\section{Click Chemistry for Drug Delivery Nanosystems}


Finn and coworkers for the labeling of mammalian cells in culture with no loss in cell viability (5 min incubations) (165), paving the way for the safer application of CuAAC in bioorthogonal chemical reporter strategies. 


\section{CONCLUSIONS}

Over the past few decades, various generations of nanosized drug delivery systems (DDS) have been developed for the controlled administration of drugs into the body. Organic synthesis has accompanied this process by providing synthetic tools for the preparation of novel materials for the construction of DDS with new properties, as well as for the efficient bioconjugation of drugs, polymers, and targeting ligands. Indeed, as this ability of putting together small building blocks into larger structures has been at the core of evolution, Nature has inspired chemists in the search for more efficient processes with production of minimal waste. In this context, and in an effort to focus the attention on the easy production of properties rather than on challenging structures, Sharpless and coworkers introduced in 2001 the concept of click chemistry. The idea was to confine the whole range of chemical transformations to a set of orthogonal processes with high thermodynamic driving force, allowing the efficient and easy transformation of "springloaded" starting materials into new substances with useful properties. Four main groups of click reactions were defined on this basis: additions to carbon-carbon multiple bonds, nucleophilic substitutions, cycloadditions, and carbonyl chemistry of the "non-aldol" type. Among them, the $\mathrm{Cu}(\mathrm{I})$-catalyzed azide-alkyne cycloaddition (CuAAC) has emerged as a paradigm in click chemistry, attracting most attention, and finding application in many areas of research. In addition, as the click concept clearly goes beyond $\mathrm{CuAAC}$, it has also triggered the search for more efficient, orthogonal, and harmless conjugation protocols of wide application, and experimental simplicity for non-specialists. Also, the introduction of the click concept has attracted a renewed interest on the use of efficient classical transformations. Firmly established reactions before the click era, such as the thiol-ene coupling, Michael addition, or Diels-Alder, are clear examples of this type.

\section{Click Chemistry for Drug Delivery Nanosystems}


As shown in this Expert Review, drug delivery has greatly benefited from click chemistry. Numerous examples of click procedures have been recently reported for the preparation and functionalization of nanosized DDS, including polymeric micelles and nanoparticles, liposomes and polymersomes, capsules, metal and silica nanoparticles, carbon nanotubes and fullerenes, or bionanoparticles. In addition, great efforts have been focused on the development of efficient bioconjugation protocols to ensure fast and high yielding functionalizations.

Nevertheless, the rapid and favorable acceptance of the click concept in drug delivery and other areas should not screen challenges still ahead. For instance, a survey of the literature reveals that in many publications entitled as "click", some of the fundamental concepts of click chemistry are being misinterpreted. Also, taken CuAAC as a leading example of a recently described click reaction, it is interesting to note that under the original conditions reported by Fokin, Sharpless and cowokers, the presence of $\mathrm{Cu}$ and reducing agents revealed detrimental for biomacromolecules (i.e., nucleic acids, proteins, polysaccharides), and hence reaction conditions have been adapted to ensure their harmless functionalization.

As a result, the complexity of nanosized DDS (where polymers, low molecular weight molecules, and biomacromolecules coexist into well-organized nanostructures) reveals as an attractive test ground with rich future for the application/development of established/new click reactions. In addition, the development of new processes with high reaction kinetics and biocompatibility within the cellular environment, the selective labeling of specific targets using wild metabolic routes, or the design of easily accessible reactive tags with long shelf-life are undoubtedly roads to ride for the biomedical applications of click chemistry, where drug delivery will find an endless source of inspiration.

\section{Click Chemistry for Drug Delivery Nanosystems}


Lallana Page 61

\section{ACKNOWLEDGEMENTS}

This work was financially supported by the Spanish Ministry of Science and Innovation (CTQ2009-10963 and CTQ2009-14146-C02-02) and the Xunta de Galicia (10CSA209021PR). 


\section{References}

1. A. S. Hoffman. The origins and evolution of "controlled" drug delivery systems. $J$. Controlled Release 132: 153-163 (2008).

2. B. Wang, T. J. Siahaan and R. Soltero. Drug delivery: Principles and applications. John Wiley \& Sons, Inc., Hoboken 2005.

3. L. van Vlerken, T. Vyas and M. Amiji. Poly(ethylene glycol)-modified nanocarriers for tumor-targeted and intracellular delivery. Pharm. Res. 24: 1405-1414 (2007).

4. J. D. Byrne, T. Betancourt and L. Brannon-Peppas. Active targeting schemes for nanoparticle systems in cancer therapeutics. Adv. Drug Delivery Rev. 60: 1615-1626 (2008).

5. H. Maeda. Tumor-selective delivery of macromolecular drugs via the EPR effect: Background and future prospects. Bioconjugate Chem. 21: 797-802 (2010).

6. I. F. Uchegbu and A. G. Schätzlein. Polymers in drug delivery.: CRC Press, Boca Raton 2006.

7. P. Anastas and N. Eghbali. Green chemistry: Principles and practice. Chem. Soc. Rev. 39: 301-312 (2010).

8. H. C. Kolb, M. G. Finn and K. B. Sharpless. Click chemistry: Diverse chemical function from a few good reactions. Angew. Chem., Int. Ed. 40: 2004-2021 (2001).

9. C. W. Tornøe, C. Christensen and M. Meldal. Peptidotriazoles on solid phase: $[1,2,3]-$ Triazoles by regiospecific copper(I)-catalyzed 1,3-dipolar cycloadditions of terminal alkynes to azides. J. Org. Chem. 67: 3057-3064 (2002).

10. V. V. Rostovtsev, L. G. Green, V. V. Fokin and K. B. Sharpless. A stepwise Huisgen cycloaddition process: Copper(I)-catalyzed regioselective "ligation" of azides and terminal alkynes. Angew. Chem., Int. Ed. 41: 2596-2599 (2002).

11. M. F. Debets, C. W. J. van der Doelen, F. P. J. T. Rutjes and F. L. van Delft. Azide: A unique dipole for metal-free bioorthogonal ligations. ChemBioChem 11: 1168-1184 (2010).

12. R. K. Iha, K. L. Wooley, A. M. Nyström, D. J. Burke, M. J. Kade and C. J. Hawker. Applications of orthogonal "click" chemistries in the synthesis of functional soft materials. Chem. Rev. 109: 5620-5686 (2009).

13. M. Meldal and C. W. Tornøe. Cu-Catalyzed azide-alkyne cycloaddition. Chem. Rev. 108: 2952-3015 (2008).

14. V. V. Fokin, Peng Wu. Catalytic azide-alkyne cycloaddition: Reactivity and applications. Aldrichimica Acta 40: 7-17 (2007).

15. M. van Dijk, D. T. S. Rijkers, R. M. J. Liskamp, C. F. van Nostrum and W. E. Hennink. Synthesis and applications of biomedical and pharmaceutical polymers via click chemistry methodologies. Bioconjugate Chem. 20: 2001-2016 (2009).

16. Q. Wang, T. R. Chan, R. Hilgraf, V. V. Fokin, K. B. Sharpless and M. G. Finn. Bioconjugation by copper(I)-catalyzed azide-alkyne [3+2] cycloaddition. J. Am. Chem. Soc. 125: 3192-3193 (2003).

17. B. Halliwell and J. M. C. Gutteridge. Role of free radicals and catalytic metal ions in human disease: An overview. Methods Enzymol. 186: 1-85 (1990).

18. S. C. Fry. Oxidative scission of plant cell wall polysaccharides by ascorbate-induced hydroxyl radicals. Biochem. J. 332: 507-515 (1998).

\section{Click Chemistry for Drug Delivery Nanosystems}


19. C. J. Duxbury, D. Cummins and A. Heise. Glaser coupling of polymers: Side-reaction in Huisgens "click" coupling reaction and opportunity for polymers with focal diacetylene units in combination with ATRP. J. Polym. Sci., Part A: Polym. Chem. 47: 3795-3802 (2009).

20. T. R. Chan, R. Hilgraf, K. B. Sharpless and V. V. Fokin. Polytriazoles as copper(I)stabilizing ligands in catalysis. Org. Lett. 6: 2853-2855 (2004).

21. W. G. Lewis, F. G. Magallon, V. V. Fokin and M. G. Finn. Discovery and characterization of catalysts for azide-alkyne cycloaddition by fluorescence quenching. $J$. Am. Chem. Soc. 126: 9152-9153 (2004).

22. V. Hong, S. I. Presolski, C. Ma and M. G. Finn. Analysis and optimization of coppercatalyzed azide-alkyne cycloaddition for bioconjugation. Angew. Chem., Int. Ed. 48: 9879-9883 (2009).

23. S. S. Gupta, J. Kuzelka, P. Singh, W. G. Lewis, M. Manchester and M. G. Finn. Accelerated bioorthogonal conjugation: A practical method for the ligation of diverse functional molecules to a polyvalent virus scaffold. Bioconjugate Chem. 16: 1572-1579 (2005).

24. N. J. Agard, J. A. Prescher and C. R. Bertozzi. A strain-promoted [3+2] azide-alkyne cycloaddition for covalent modification of biomolecules in living systems. J. Am. Chem. Soc. 126: 15046-15047 (2004).

25. V. Hong, A. K. Udit, R. A. Evans and M. G. Finn. Electrochemically protected copper(I)catalyzed azide-alkyne cycloaddition. ChemBioChem 9: 1481-1486 (2008).

26. R. Kumar, A. H. El-Sagheer, J. Tumpane, P. Lincoln, L. M. Wilhelmsson and T. Brown. Template-directed oligonucleotide strand ligation, covalent intramolecular DNA circularization and catenation using click chemistry. J. Am. Chem. Soc. 129: 6859-6864 (2007).

27. J. M. Baskin and C. R. Bertozzi. Copper-free click chemistry: Bioorthogonal reagents for tagging azides. Aldrichimica Acta 43: 15-23 (2010).

28. J. M. Baskin, J. A. Prescher, S. T. Laughlin, N. J. Agard, P. V. Chang, I. A. Miller, A. Lo, J. A. Codelli and C. R. Bertozzi. Copper-free click chemistry for dynamic in vivo imaging. Proc. Natl. Acad. Sci. U.S.A. 104: 16793-16797 (2007).

29. J. A. Codelli, J. M. Baskin, N. J. Agard and C. R. Bertozzi. Second-generation difluorinated cyclooctynes for copper-free click chemistry. J. Am. Chem. Soc. 130: 11486-11493 (2008).

30. X. Ning, J. Guo, M. A. Wolfert and G.-J. Boons. Visualizing metabolically labeled glycoconjugates of living cells by copper-free and fast Huisgen cycloadditions. Angew. Chem., Int. Ed. 47: 2253-2255 (2008).

31. M. F. Debets, S. S. van Berkel, S. Schoffelen, F. P. J. T. Rutjes, J. C. M. van Hest and F. L. van Delft. Aza-dibenzocyclooctynes for fast and efficient enzyme PEGylation via copper-free (3+2) cycloaddition. Chem. Commun. 46: 97-99 (2010).

32. J. C. Jewett, E. M. Sletten and C. R. Bertozzi. Rapid Cu-free click chemistry with readily synthesized biarylazacyclooctynones. J. Am. Chem. Soc. 132: 3688-3690 (2010).

33. J. Dommerholt, S. Schmidt, R. Temming, L. J. A. Hendriks, F. P. J. T. Rutjes, J. C. M. van Hest, D. J. Lefeber, P. Friedl and F. L. van Delft. Readily accessible bicyclononynes for bioorthogonal labeling and three-dimensional imaging of living cells. Angew. Chem., Int. Ed. 49: 9422-9425 (2010).

\section{Click Chemistry for Drug Delivery Nanosystems}


34. A. A. Poloukhtine, N. E. Mbua, M. A. Wolfert, G.-J. Boons and V. V. Popik. Selective labeling of living cells by a photo-triggered click reaction. J. Am. Chem. Soc. 131: 15769-15776 (2009).

35. O. C. Farokhzad and R. Langer. Impact of nanotechnology on drug delivery. ACS Nano 3: 16-20 (2009).

36. K. Kataoka, A. Harada and Y. Nagasaki. Block copolymer micelles for drug delivery: Design, characterization and biological significance. Adv. Drug Delivery Rev. 47: 113131 (2001).

37. P. De, S. R. Gondi and B. S. Sumerlin. Folate-conjugated thermoresponsive block copolymers: Highly efficient conjugation and solution self-assembly. Biomacromolecules 9: $1064-1070$ (2008).

38. N. Xu, R. Wang, F.-S. Du and Z.-C. Li. Synthesis of amphiphilic biodegradable glycocopolymers based on poly( $\varepsilon$-caprolactone) by ring-opening polymerization and click chemistry. J. Polym. Sci., Part A: Polym. Chem. 47: 3583-3594 (2009).

39. G. Chen, S. Amajjahe and M. H. Stenzel. Synthesis of thiol-linked neoglycopolymers and thermo-responsive glycomicelles as potential drug carrier. Chem. Commun.: 1198-1200 (2009).

40. Y. Yang, C. Hua and C.-M. Dong. Synthesis, self-assembly, and in vitro doxorubicin release behavior of dendron-like/linear/dendron-like poly( $\varepsilon$-caprolactone)- $b$ poly(ethylene glycol)- $b$-poly( $\varepsilon$-caprolactone) triblock copolymers. Biomacromolecules 10: 2310-2318 (2009).

41. S.-M. Peng, Y. Chen, C. Hua and C.-M. Dong. Dendron-like polypeptide/linear poly(ethylene oxide) biohybrids with both asymmetrical and symmetrical topologies synthesized via the combination of click chemistry and ring-opening polymerization. Macromolecules 42: 104-113 (2009).

42. C. Hua, S.-M. Peng and C.-M. Dong. Synthesis and characterization of linear-dendronlike poly( $\varepsilon$-caprolactone)- $b$-poly(ethylene oxide) copolymers via the combination of ringopening polymerization and click chemistry. Macromolecules 41: 6686-6695 (2008).

43. G. M. Soliman, R. Sharma, A. O. Choi, S. K. Varshney, F. M. Winnik, A. K. Kakkar and D. Maysinger. Tailoring the efficacy of nimodipine drug delivery using nanocarriers based on $\mathrm{A}_{2} \mathrm{~B}$ miktoarm star polymers. Biomaterials 31: 8382-8392 (2010).

44. C.-Y. Quan, J.-X. Chen, H.-Y. Wang, C. Li, C. Chang, X.-Z. Zhang and R.-X. Zhuo. Core-shell nanosized assemblies mediated by the $\alpha-\beta$ cyclodextrin dimer with a tumortriggered targeting property. ACS Nano 4: 4211-4219 (2010).

45. J.-F. Lutz, S. Pfeifer and Z. Zarafshani. "In situ" functionalization of thermoresponsive polymeric micelles using the "click" cycloaddition of azides and alkynes. QSAR Comb. Sci. 26: 1151-1158 (2007).

46. J. Nicolas and P. Couvreur. Synthesis of poly(alkyl cyanoacrylate)-based colloidal nanomedicines. Wiley Interdiscip. Rev. Nanomed. Nanobiotechnol. 1: 111-127 (2009).

47. J. Nicolas, F. Bensaid, D. Desmaële, M. Grogna, C. Detrembleur, K. Andrieux and P. Couvreur. Synthesis of highly functionalized poly(alkyl cyanoacrylate) nanoparticles by means of click chemistry. Macromolecules 41: 8418-8428 (2008).

48. J. Lu, M. Shi and M. S. Shoichet. Click chemistry functionalized polymeric nanoparticles target corneal epithelial cells through RGD-cell surface receptors. Bioconjugate Chem. 20: 87-94 (2009).

\section{Click Chemistry for Drug Delivery Nanosystems}


49. M. Shi, J. H. Wosnick, K. Ho, A. Keating and M. S. Shoichet. Immuno-polymeric nanoparticles by Diels-Alder chemistry. Angew. Chem., Int. Ed. 46: 6126-6131 (2007).

50. M. Shi, K. Ho, A. Keating and M. S. Shoichet. Doxorubicin-conjugated immunonanoparticles for intracellular anticancer drug delivery. Adv. Funct. Mater. 19: 16891696 (2009).

51. Z. An, W. Tang, M. Wu, Z. Jiao and G. D. Stucky. Heterofunctional polymers and coreshell nanoparticles via cascade aminolysis/Michael addition and alkyne-azide click reaction of RAFT polymers. Chem. Commun.: 6501-6503 (2008).

52. P. A. Bertin, K. J. Watson and S. T. Nguyen. Indomethacin-containing nanoparticles derived from amphiphilic polynorbornene: A model ROMP-based drug encapsulation system. Macromolecules 37: 8364-8372 (2004).

53. P. A. Bertin, D. Smith and S. T. Nguyen. High-density doxorubicin-conjugated polymeric nanoparticles via ring-opening metathesis polymerization. Chem. Commun.: 3793-3795 (2005).

54. S. A. Krovi, D. Smith and S. T. Nguyen. "Clickable" polymer nanoparticles: A modular scaffold for surface functionalization. Chem. Commun. 46: 5277-5279 (2010).

55. Y. Han, Q. Shi, J. Hu, Q. Du, X. Chen and X. Jing. Grafting BSA onto poly[(L-lactide)co-carbonate] microspheres by click chemistry. Macromol. Biosci. 8: 638-644 (2008).

56. Q. Shi, X. Chen, T. Lu and X. Jing. The immobilization of proteins on biodegradable polymer fibers via click chemistry. Biomaterials 29: 1118-1126 (2008).

57. Q. Shi, Y. Huang, X. Chen, M. Wu, J. Sun and X. Jing. Hemoglobin conjugated micelles based on triblock biodegradable polymers as artificial oxygen carriers. Biomaterials 30: 5077-5085 (2009).

58. X. Wang, L. Liu, Y. Luo and H. Zhao. Bioconjugation of biotin to the interfaces of polymeric micelles via in situ click chemistry. Langmuir 25: 744-750 (2009).

59. A. Harada and K. Kataoka. Formation of polyion complex micelles in an aqueous milieu from a pair of oppositely-charged block copolymers with poly(ethylene glycol) segments. Macromolecules 28: 5294-5299 (1995).

60. A. V. Kabanov, T. K. Bronich, V. A. Kabanov, K. Yu and A. Eisenberg. Soluble stoichiometric complexes from poly(N-ethyl-4-vinylpyridinium) cations and poly(ethylene oxide)-block-polymethacrylate anions. Macromolecules 29: 6797-6802 (1996).

61. A. Sousa-Herves, E. Fernandez-Megia and R. Riguera. Synthesis and supramolecular assembly of clicked anionic dendritic polymers into polyion complex micelles. Chem. Commun.: 3136-3138 (2008).

62. J. Zhang, Y. Zhou, Z. Zhu, Z. Ge and S. Liu. Polyion complex micelles possessing thermoresponsive coronas and their covalent core stabilization via "click" chemistry. Macromolecules 41: 1444-1454 (2008).

63. R. K. O'Reilly, C. J. Hawker and K. L. Wooley. Cross-linked block copolymer micelles: Functional nanostructures of great potential and versatility. Chem. Soc. Rev. 35: 10681083 (2006).

64. E. S. Read and S. P. Armes. Recent advances in shell cross-linked micelles. Chem. Commun.: 3021-3035 (2007).

65. M. J. Joralemon, R. K. O'Reilly, C. J. Hawker and K. L. Wooley. Shell click-crosslinked (SCC) nanoparticles: A new methodology for synthesis and orthogonal functionalization. J. Am. Chem. Soc. 127: 16892-16899 (2005).

\section{Click Chemistry for Drug Delivery Nanosystems}


66. R. K. O'Reilly, M. J. Joralemon, C. J. Hawker and K. L. Wooley. Preparation of orthogonally-functionalized core click cross-linked nanoparticles. New J. Chem. 31: 718724 (2007).

67. X. Jiang, J. Zhang, Y. Zhou, J. Xu and S. Liu. Facile preparation of core-crosslinked micelles from azide-containing thermoresponsive double hydrophilic diblock copolymer via click chemistry. J. Polym. Sci., Part A: Polym. Chem. 46: 860-871 (2008).

68. X. Jiang, G. Zhang, R. Narain and S. Liu. Fabrication of two types of shell-cross-linked micelles with "inverted" structures in aqueous solution from schizophrenic water-soluble ABC triblock copolymer via click chemistry. Langmuir 25: 2046-2054 (2009).

69. A. B. J. Withey, G. Chen, T. L. U. Nguyen and M. H. Stenzel. Macromolecular cobalt carbonyl complexes encapsulated in a click-cross-linked micelle structure as a nanoparticle to deliver cobalt pharmaceuticals. Biomacromolecules 10: 3215-3226 (2009).

70. E. Lallana, E. Fernandez-Megia and R. Riguera. Surpassing the use of copper in the click functionalization of polymeric nanostructures: A strain-promoted approach. J. Am. Chem. Soc. 131: 5748-5750 (2009).

71. R. R. Sawant and V. P. Torchilin. Liposomes as "smart" pharmaceutical nanocarriers. Soft Matter 6: 4026-4044 (2010).

72. F. S. Hassane, B. Frisch and F. Schuber. Targeted liposomes: Convenient coupling of ligands to preformed vesicles using "click chemistry". Bioconjugate Chem. 17: 849-854 (2006).

73. S. Cavalli, A. R. Tipton, M. Overhand and A. Kros. The chemical modification of liposome surfaces via a copper-mediated [3 +2$]$ azide-alkyne cycloaddition monitored by a colorimetric assay. Chem. Commun.: 3193-3195 (2006).

74. A. Kumar, U. J. Erasquin, G. Qin, K. Li and C. Cai. "Clickable", polymerized liposomes as a versatile and stable platform for rapid optimization of their peripheral compositions. Chem. Commun. 46: 5746-5748 (2010).

75. B. M. Discher, Y.-Y. Won, D. S. Ege, J. C.-M. Lee, F. S. Bates, D. E. Discher and D. A. Hammer. Polymersomes: Tough vesicles made from diblock copolymers. Science 284: 1143-1146 (1999).

76. C. LoPresti, H. Lomas, M. Massignani, T. Smart and G. Battaglia. Polymersomes: nature inspired nanometer sized compartments. J. Mater. Chem. 19: 3576-3590 (2009).

77. J. A. Opsteen, R. P. Brinkhuis, R. L. M. Teeuwen, D. W. P. M. Löwik and J. C. M. van Hest. "Clickable" polymersomes. Chem. Commun.: 3136-3138 (2007).

78. B. Li, A. L. Martin and E. R. Gillies. Multivalent polymer vesicles via surface functionalization. Chem. Commun.: 5217-5219 (2007).

79. A. L. Martin, B. Li and E. R. Gillies. Surface functionalization of nanomaterials with dendritic groups: toward enhanced binding to biological targets. J. Am. Chem. Soc. 131: 734-741 (2009).

80. S.-M. Lee, H. Chen, C. M. Dettmer, T. V. O'Halloran and S. T. Nguyen. Polymer-caged lipsomes: A pH-responsive delivery system with high stability. J. Am. Chem. Soc. 129: 15096-15097 (2007).

81. S.-M. Lee, H. Chen, T. V. O'Halloran and S. T. Nguyen. "Clickable" polymer-caged nanobins as a modular drug delivery platform. J. Am. Chem. Soc. 131: 9311-9320 (2009). 
82. J. F. Quinn, A. P. R. Johnston, G. K. Such, A. N. Zelikin and F. Caruso. Next generation, sequentially assembled ultrathin films: Beyond electrostatics. Chem. Soc. Rev. 36: 707718 (2007).

83. G. K. Such, E. Tjipto, A. Postma, A. P. R. Johnston and F. Caruso. Ultrathin, responsive polymer click capsules. Nano Lett. 7: 1706-1710 (2007).

84. C. J. Ochs, G. K. Such, B. Städler and F. Caruso. Low-fouling, biofunctionalized, and biodegradable click capsules. Biomacromolecules 9: 3389-3396 (2008).

85. B. G. De Geest, W. Van Camp, F. E. Du Prez, S. C. De Smedt, J. Demeester and W. E. Hennink. Degradable multilayer films and hollow capsules via a "click" strategy.

Macromol. Rapid Commun. 29: 1111-1118 (2008).

86. B. G. De Geest, W. Van Camp, F. E. Du Prez, S. C. De Smedt, J. Demeester and W. E. Hennink. Biodegradable microcapsules designed via 'click' chemistry. Chem. Commun.: 190-192 (2008).

87. L. A. Connal, C. R. Kinnane, A. N. Zelikin and F. Caruso. Stabilization and functionalization of polymer multilayers and capsules via thiol-ene click chemistry. Chem. Mater. 21: 576-578 (2009).

88. M. M. J. Kamphuis, A. P. R. Johnston, G. K. Such, H. H. Dam, R. A. Evans, A. M. Scott, E. C. Nice, J. K. Heath and F. Caruso. Targeting of cancer cells using clickfunctionalized polymer capsules. J. Am. Chem. Soc. 132: 15881-15883 (2010).

89. C. J. Ochs, G. K. Such, Y. Yan, M. P. van Koeverden and F. Caruso. Biodegradable click capsules with engineered drug-loaded multilayers. ACS Nano 4: 1653-1663 (2010).

90. D. Kim, E. Kim, J. Lee, S. Hong, W. Sung, N. Lim, C. G. Park and K. Kim. Direct synthesis of polymer nanocapsules: Self-assembly of polymer hollow spheres through Irreversible covalent bond formation. J. Am. Chem. Soc. 132: 9908-9919 (2010).

91. D. R. Breed, R. Thibault, F. Xie, Q. Wang, C. J. Hawker and D. J. Pine. Functionalization of polymer microspheres using click chemistry. Langmuir 25: 4370-4376 (2009).

92. A. S. Goldmann, A. Walther, L. Nebhani, R. Joso, D. Ernst, K. Loos, C. BarnerKowollik, L. Barner and A. H. E. Müller. Surface modification of poly(divinylbenzene) microspheres via thiol-ene chemistry and alkyne-azide click reactions. Macromolecules 42: 3707-3714 (2009).

93. M. Arruebo, R. Fernández-Pacheco, M. R. Ibarra and J. Santamaría. Magnetic nanoparticles for drug delivery. Nano Today 2: 22-32 (2007).

94. R. A. Sperling, P. Rivera Gil, F. Zhang, M. Zanella and W. J. Parak. Biological applications of gold nanoparticles. Chem. Soc. Rev. 37: 1896-1908 (2008).

95. E. Boisselier and D. Astruc. Gold nanoparticles in nanomedicine: Preparations, imaging, diagnostics, therapies and toxicity. Chem. Soc. Rev. 38: 1759-1782 (2009).

96. D. A. Fleming, C. J. Thode and M. E. Williams. Triazole cycloaddition as a general route for functionalization of Au nanoparticles. Chem. Mater. 18: 2327-2334 (2006).

97. E. Boisselier, L. Salmon, J. Ruiz and D. Astruc. How to very efficiently functionalize gold nanoparticles by "click" chemistry. Chem. Commun.: 5788-5790 (2008).

98. J. L. Brennan, N. S. Hatzakis, T. R. Tshikhudo, N. Dirvianskyte, V. Razumas, S. Patkar, J. Vind, A. Svendsen, R. J. M. Nolte, A. E. Rowan and M. Brust. Bionanoconjugation via click chemistry: The creation of functional hybrids of lipases and gold nanoparticles.

Bioconjugate Chem. 17: 1373-1375 (2006).

\section{Click Chemistry for Drug Delivery Nanosystems}


99. Y.-P. Kim, W. L. Daniel, Z. Xia, H. Xie, C. A. Mirkin and J. Rao. Bioluminescent nanosensors for protease detection based upon gold nanoparticle-luciferase conjugates. Chem. Commun. 46: 76-78 (2010).

100. M. Fischler, A. Sologubenko, J. Mayer, G. Clever, G. Burley, J. Gierlich, T. Carell and U. Simon. Chain-like assembly of gold nanoparticles on artificial DNA templates via "click chemistry". Chem. Commun.: 169-171 (2008).

101. M.-X. Zhang, B.-H. Huang, X.-Y. Sun and D.-W. Pang. Clickable gold nanoparticles as the building block of nanobioprobes. Langmuir 26: 10171-10176 (2010).

102. H. Li, Y. Yao, C. Han and J. Zhan. Triazole-ester modified silver nanoparticles: Click synthesis and $\mathrm{Cd}^{2+}$ colorimetric sensing. Chem. Commun.: 4812-4814 (2009).

103. Y. Yao, D. Tian and H. Li. Cooperative binding of bifunctionalized and click-synthesized silver nanoparticles for colorimetric $\mathrm{Co}^{2+}$ sensing. ACS Appl. Mater. Interfaces 2: 684$690(2010)$.

104. E. Drockenmuller, I. Colinet, D. Damiron, F. Gal, H. Perez and G. Carrot. Efficient approaches for the surface modification of platinum nanoparticles via click chemistry. . Macromolecules 43: $9371-9375$ (2010).

105. S. Koenig and V. Chechik. Shell cross-linked Au nanoparticles. Langmuir 22: 5168-5173 (2006).

106. P. J. Costanzo and F. L. Beyer. Thermally driven assembly of nanoparticles in polymer matrices. Macromolecules 40: 3996-4001 (2007).

107. S. Mornet, S. Vasseur, F. Grasset and E. Duguet. Magnetic nanoparticle design for medical diagnosis and therapy. J. Mater. Chem. 14: 2161-2175 (2004).

108. M. A. White, J. A. Johnson, J. T. Koberstein and N. J. Turro. Toward the syntheses of universal ligands for metal oxide surfaces: Controlling surface functionality through click chemistry. J. Am. Chem. Soc. 128: 11356-11357 (2006).

109. P. C. Lin, S. H. Ueng, S. C. Yu, M. D. Jan, A. K. Adak, C. C. Yu and C. C. Lin. Surface modification of magnetic nanoparticle via $\mathrm{Cu}(\mathrm{I})$-catalyzed alkyne-azide [2 + 3] cycloaddition. Org. Lett. 9: 2131-2134 (2007).

110. P.-C. Lin, S.-H. Ueng, M.-C. Tseng, J.-L. Ko, K.-T. Huang, S.-C. Yu, A. K. Adak, Y.-J. Chen and C.-C. Lin. Site-specific protein modification through $\mathrm{Cu}^{\mathrm{I}}$-catalyzed 1,2,3triazole formation and its implementation in protein microarray fabrication. Angew. Chem., Int. Ed. 45: 4286-4290 (2006).

111. L. Polito, D. Monti, E. Caneva, E. Delnevo, G. Russo and D. Prosperi. One-step bioengineering of magnetic nanoparticles via a surface diazo transfer/azide-alkyne click reaction sequence. Chem. Commun.: 621-623 (2008).

112. P. T. Nyffeler, C.-H. Liang, K. M. Koeller and C.-H. Wong. The chemistry of amineazide interconversion: Catalytic diazotransfer and regioselective azide reduction. J. Am. Chem. Soc. 124: 10773-10778 (2002).

113. J. I. Cutler, D. Zheng, X. Xu, D. A. Giljohann and C. A. Mirkin. Polyvalent oligonucleotide iron oxide nanoparticle "click" conjugates. Nano Lett. 10: 1477-1480 (2010).

114. D. R. Elias, Z. Cheng and A. Tsourkas. An intein-mediated site-specific click conjugation strategy for improved tumor targeting of nanoparticle systems. Small 6: 2460-2468 (2010).

\section{Click Chemistry for Drug Delivery Nanosystems}


115. K. Hayashi, M. Moriya, W. Sakamoto and T. Yogo. Chemoselective synthesis of folic acid-functionalized magnetite nanoparticles via click chemistry for magnetic hyperthermia. Chem. Mater. 21: 1318-1325 (2009).

116. K. Hayashi, K. Ono, H. Suzuki, M. Sawada, M. Moriya, W. Sakamoto and T. Yogo. High-frequency, magnetic-field-responsive drug release from magnetic nanoparticle/organic hybrid based on hyperthermic effect. ACS Appl. Mater. Interfaces 2: 1903-1911 (2010).

117. S. Santra, C. Kaittanis, J. Grimm and J. M. Perez. Drug/dye-loaded, multifunctional iron oxide nanoparticles for combined targeted cancer therapy and dual optical/magnetic resonance imaging. Small 5: 1862-1868 (2009).

118. N. K. Devaraj, E. J. Keliher, G. M. Thurber, M. Nahrendorf and R. Weissleder. ${ }^{18} \mathrm{~F}$ labeled nanoparticles for in vivo PET-CT imaging. Bioconjugate Chem. 20: 397-401 (2009).

119. M. Nahrendorf, E. Keliher, B. Marinelli, P. Waterman, P. F. Feruglio, L. Fexon, M. Pivovarov, F. K. Swirski, M. J. Pittet, C. Vinegoni and R. Weissleder. Hybrid PEToptical imaging using targeted probes. Proc. Natl. Acad. Sci. U.S.A. 107: 7910-7915 (2010).

120. G. von Maltzahn, Y. Ren, J.-H. Park, D.-H. Min, V. R. Kotamraju, J. Jayakumar, V. Fogal, M. J. Sailor, E. Ruoslahti and S. N. Bhatia. In vivo tumor cell targeting with "click" nanoparticles. Bioconjugate Chem. 19: 1570-1578 (2008).

121. K. Hayashi, K. Ono, H. Suzuki, M. Sawada, M. Moriya, W. Sakamoto and T. Yogo. Onepot biofunctionalization of magnetic nanoparticles via thiol-ene click reaction for magnetic hyperthermia and magnetic resonance imaging. Chem. Mater. 22: 3768-3772 (2010).

122. R. D. Rutledge, C. L. Warner, J. W. Pittman, R. S. Addleman, M. Engelhard, W. Chouyyok and M. G. Warner. Thiol-ene induced diphosphonic acid functionalization of superparamagnetic iron oxide nanoparticles. Langmuir 26: 12285-12292 (2010).

123. X. Michalet, F. F. Pinaud, L. A. Bentolila, J. M. Tsay, S. Doose, J. J. Li, G. Sundaresan, A. M. Wu, S. S. Gambhir and S. Weiss. Quantum dots for live cells, in vivo imaging, and diagnostics. Science 307: 538-544 (2005).

124. W. H. Binder, R. Sachsenhofer, C. J. Straif and R. Zirbs. Surface-modified nanoparticles via thermal and $\mathrm{Cu}(\mathrm{I})$-mediated "click" chemistry: Generation of luminescent $\mathrm{CdSe}$ nanoparticles with polar ligands guiding supramolecular recognition. J. Mater. Chem. 17: 2125-2132 (2007).

125. A. Bernardin, A. Cazet, L. Guyon, P. Delannoy, F. Vinet, D. Bonnaffé and I. Texier. Copper-free click chemistry for highly luminescent quantum dot conjugates: Application to in vivo metabolic imaging. Bioconjugate Chem. 21: 583-588 (2010).

126. H.-S. Han, N. K. Devaraj, J. Lee, S. A. Hilderbrand, R. Weissleder and M. G. Bawendi. Development of a bioorthogonal and highly efficient conjugation method for quantum dots using tetrazine-norbornene cycloaddition. J. Am. Chem. Soc. 132: 7838-7839 (2010).

127. I. I. Slowing, B. G. Trewyn, S. Giri and V. S. Y. Lin. Mesoporous silica nanoparticles for drug delivery and biosensing applications. Adv. Funct. Mater. 17: 1225-1236 (2007).

128. R. Ranjan and W. J. Brittain. Combination of living radical polymerization and click chemistry for surface modification. Macromolecules 40: 6217-6223 (2007).

\section{Click Chemistry for Drug Delivery Nanosystems}


129. R. Ranjan and W. J. Brittain. Tandem RAFT polymerization and click chemistry: An efficient approach to surface modification. Macromol. Rapid Commun. 28: 2084-2089 (2007).

130. J. Zhang, X. Wang, D. Wu, L. Liu and H. Zhao. Bioconjugated Janus particles prepared by in situ click chemistry. Chem. Mater. 21: 4012-4018 (2009).

131. M. Müllner, A. Schallon, A. Walther, R. Freitag and A. H. E. Müller. Clickable, biocompatible, and fluorescent hybrid nanoparticles for intracellular delivery and optical imaging. Biomacromolecules 11: 390-396 (2010).

132. P. Kele, G. Mezö, D. Achatz and O. S. Wolfbeis. Dual labeling of biomolecules by using click chemistry: A sequential approach. Angew. Chem., Int. Ed. 48: 344-347 (2009).

133. D. E. Achatz, G. Mezö, P. Kele and O. S. Wolfbeis. Probing the activity of matrix metalloproteinase II with a sequentially click-labeled silica nanoparticle FRET probe. ChemBioChem 10: 2316-2320 (2009).

134. A. Schlossbauer, S. Warncke, P. M. E. Gramlich, J. Kecht, A. Manetto, T. Carell and T. Bein. A programmable DNA-based molecular valve for colloidal mesoporous silica. Angew. Chem., Int. Ed. 49: 4734-4737 (2010).

135. D. M. Guldi, G. M. A. Rahman, V. Sgobba and C. Ehli. Multifunctional molecular carbon materials-from fullerenes to carbon nanotubes. Chem. Soc. Rev. 35: 471-487 (2006).

136. A. Bianco, K. Kostarelos, C. D. Partidos and M. Prato. Biomedical applications of functionalised carbon nanotubes. Chem. Commun.: 571-577 (2005).

137. R. Partha and J. L. Conyers. Biomedical applications of functionalized fullerene-based nanomaterials. Int. J. Nanomed. 4: 261-275 (2009).

138. H. Li, F. Cheng, A. M. Duft and A. Adronov. Functionalization of single-walled carbon nanotubes with well-defined polystyrene by "click" coupling. J. Am. Chem. Soc. 127: 14518-14524 (2005).

139. Z. Guo, L. Liang, J.-J. Liang, Y.-F. Ma, X.-Y. Yang, D.-M. Ren, Y.-S. Chen and J.-Y. Zheng. Covalently $\beta$-cyclodextrin modified single-walled carbon nanotubes: A novel artificial receptor synthesized by "click" chemistry. J. Nanopart. Res. 10: 1077-1083 (2008).

140. Y. Zhang, H. He and C. Gao. Clickable macroinitiator strategy to build amphiphilic polymer brushes on carbon nanotubes. Macromolecules 41: 9581-9594 (2008).

141. R. Voggu, S. Pal, S. K. Pati and C. N. R. Rao. Semiconductor to metal transition in SWNTs caused by interaction with gold and platinum nanoparticles. J. Phys.: Condens. Matter 20: 215211 (2008).

142. H. He, Y. Zhang, C. Gao and J. Y. Wu. 'Clicked' magnetic nanohybrids with a soft polymer interlayer. Chem. Commun.: 1655-1657 (2009).

143. T. Palacin, H. L. Khanh, B. Jousselme, P. Jegou, A. Filoramo, C. Ehli, D. M. Guldi and S. Campidelli. Efficient functionalization of carbon nanotubes with porphyrin dendrons via click chemistry. J. Am. Chem. Soc. 131: 15394-15402 (2009).

144. P. Wu, X. Chen, N. Hu, U. C. Tam, O. Blixt, A. Zettl and C. R. Bertozzi. Biocompatible carbon nanotubes generated by functionalization with glycodendrimers. Angew. Chem. Inter. Ed. 47: 5022-5025 (2008).

145. H. Isobe, K. Cho, N. Solin, D. B. Werz, P. H. Seeberger and E. Nakamura. Synthesis of fullerene glycoconjugates via a copper-catalyzed Huisgen cycloaddition reaction. Org. Lett. 9: 4611-4614 (2007).

\section{Click Chemistry for Drug Delivery Nanosystems}


146. J. Iehl, R. Pereira de Freitas, B. Delavaux-Nicot and J.-F. Nierengarten. Click chemistry for the efficient preparation of functionalized [60]fullerene hexakis-adducts. Chem. Commun.: 2450-2452 (2008).

147. J. Iehl and J.-F. Nierengarten. A click-click approach for the preparation of functionalized [5:1]-hexaadducts of C60. Chem. Eur. J. 15: 7306-7309 (2009).

148. P. Compain, C. Decroocq, J. Iehl, M. Holler, D. Hazelard, T. Mena Barragán, C. O. Mellet and J.-F. Nierengarten. Glycosidase inhibition with fullerene iminosugar balls: A dramatic multivalent effect. Angew. Chem., Int. Ed. 49: 5753-5756 (2010).

149. W.-B. Zhang, Y. Tu, R. Ranjan, R. M. van Horn, S. Leng, J. Wang, M. J. Polce, C. Wesdemiotis, R. P. Quirk, G. R. Newkome and S. Z. D. Cheng. "Clicking" fullerene with polymers: Synthesis of [60]fullerene end-capped polystyrene. Macromolecules 41: 515517 (2008).

150. N. F. Steinmetz, V. Hong, E. D. Spoerke, P. Lu, K. Breitenkamp, M. G. Finn and M. Manchester. Buckyballs meet viral nanoparticles: Candidates for biomedicine. J. Am. Chem. Soc. 131: 17093-17095 (2009).

151. M. A. Cremonini, L. Lunazzi, G. Placucci and P. J. Krusic. Addition of alkylthiyl and alkoxy radicals to C60 studied by ESR. J. Org. Chem. 58: 4735-4738 (1993).

152. J. Iehl and J.-F. Nierengarten. Sequential copper catalyzed alkyne-azide and thiol-ene click reactions for the multiple functionalization of fullerene hexaadducts. Chem. Commun. 46: 4160-4162 (2010).

153. L. A. Lee and Q. Wang. Adaptations of nanoscale viruses and other protein cages for medical applications. Nanomedicine: NBM 2: 137-149 (2006).

154. S. S. Gupta, K. S. Raja, E. Kaltgrad, E. Strable and M. G. Finn. Virus-glycopolymer conjugates by copper(I) catalysis of atom transfer radical polymerization and azidealkyne cycloaddition. Chem. Commun.: 4315-4317 (2005).

155. J. D. E. Prasuhn, R. M. Yeh, A. Obenaus, M. Manchester and M. G. Finn. Viral MRI contrast agents: Coordination of $\mathrm{Gd}$ by native virions and attachment of $\mathrm{Gd}$ complexes by azide-alkyne cycloaddition. Chem. Commun.: 1269-1271 (2007).

156. E. Kaltgrad, S. Sen Gupta, S. Punna, C.-Y. Huang, A. Chang, C.-H. Wong, M. G. Finn and O. Blixt. Anti-carbohydrate antibodies elicited by polyvalent display on a viral scaffold. ChemBioChem 8: 1455-1462 (2007).

157. G. Destito, R. Yeh, C. S. Rae, M. G. Finn and M. Manchester. Folic acid-mediated targeting of Cowpea mosaic virus particles to tumor cells. Chem. Biol. 14: 1152-1162 (2007).

158. E. Strable, D. E. Prasuhn, A. K. Udit, S. Brown, A. J. Link, J. T. Ngo, G. Lander, J. Quispe, C. S. Potter, B. Carragher, D. A. Tirrell and M. G. Finn. Unnatural amino acid incorporation into virus-like particles. Bioconjugate Chem. 19: 866-875 (2008).

159. N. F. Steinmetz, M. E. Mertens, R. E. Taurog, J. E. Johnson, U. Commandeur, R. Fischer and $\mathrm{M}$. Manchester. Potato virus $\mathrm{X}$ as a novel platform for potential biomedical applications. Nano Lett. 10: 305-312 (2010).

160. Q. Zeng, T. Li, B. Cash, S. Li, F. Xie and Q. Wang. Chemoselective derivatization of a bionanoparticle by click reaction and ATRP reaction. Chem. Commun.: 1453-1455 (2007).

161. P. S. Banerjee, P. Ostapchuk, P. Hearing and I. Carrico. Chemoselective attachment of small molecule effector functionality to human adenoviruses facilitates gene delivery to cancer cells. J. Am. Chem. Soc. 132: 13615-13617 (2010).

\section{Click Chemistry for Drug Delivery Nanosystems}


162. M. J. Abedin, L. Liepold, P. Suci, M. Young and T. Douglas. Synthesis of a cross-linked branched polymer network in the interior of a protein cage. J. Am. Chem. Soc. 131: 43464354 (2009).

163. J. Lucon, M. J. Abedin, M. Uchida, L. Liepold, C. C. Jolley, M. Young and T. Douglas. A click chemistry based coordination polymer inside small heat shock protein. Chem. Commun. 46: 264-266 (2010).

164. D. Banerjee, A. P. Liu, N. R. Voss, S. L. Schmid and M. G. Finn. Multivalent display and receptor-mediated endocytosis of transferrin on virus-like particles. ChemBioChem 11: 1273-1279 (2010).

165. V. Hong, N. F. Steinmetz, M. Manchester and M. G. Finn. Labeling live cells by coppercatalyzed alkyne-azide click chemistry. Bioconjugate Chem. 21: 1912-1916 (2010). 


\section{Figure Legends}

Figure 1 Synthetic sequence reported by Sharpless and coworkers to highlight the four groups of click reactions originally proposed $(\operatorname{ref}(8))$.

Figure 2 Thermal a) and $\mathrm{Cu}(\mathrm{I})$-catalyzed b) versions of the Huisgen 1,3-dipolar AAC.

Figure $3 \mathrm{Cu}(\mathrm{I})$-chelating ligands commonly employed in $\mathrm{CuAAC}$ bioconjugation.

Figure 4 Functionalization of bacteriophage $\mathrm{Q} \beta$ via different $\mathrm{CuAAC}$ bioconjugation protocols: $\mathrm{Cu}(\mathrm{I}), \mathrm{BPDS}, \mathrm{O}_{2}$-free; $\mathrm{Cu}(\mathrm{II}), \mathrm{BPDS}$, electrolysis; $\mathrm{Cu}(\mathrm{II})$, ascorbate, THPTA, aminoguanidine (ref (25) and (22)).

Figure 5 SPAAC a), and various activated cyclooctyne derivatives used in bioconjugation $b$ ).

Figure 6 RAFT/TEC synthetic strategy for the preparation of glucose functionalized copolymers and thermoresponsive micelles. [LCST $=$ lower critical solution temperature]. Reprinted with permission from ref (39).

Figure 7 Synthesis of dendron-like PCL- $b$-PEG- $b$-dendron-like PCL triblock copolymers by $\mathrm{CuAAC}$ and self-assembly into flower-like micelles. Reprinted with permission from ref (40). 
Figure 8 Formation and cellular behavior of core-shell micelles with switchable tumor-triggered targeting properties. [NAS ( $N$-acroyloxysuccinimide); MPEG (methoxy-PEG); Ad (adamantyl); NCCM (non-covalently connected micelle)]. Reprinted with permission from ref (44).

Figure 9 General approaches to the preparation of functionalized PACA micelles. Reprinted with permission from ref (47).

Figure 10 Self-assembly of P(TMCC-co-LA)-g-PEG-furan and preparation of immuno-micelles via Diels-Alder functionalization with maleimide-modified antibodies. Reprinted with permission from ref (49).

Figure 11 Preparation of heterodifunctionalized core-shell micelles via sequential aminolysis/Michael addition and CuAAC functionalizations. Reprinted with permission from ref $(51)$.

Figure 12 Conjugation of hemoglobin to the core surface of PLA- $b$-PMPC- $b$-PEG micelles via CuAAC. [CO-Hb (carbonylhemoglobin)]. Reprinted with permission from ref (57).

Figure $13 \mathrm{CuAAC}$ functionalization of PEG-dendritic block copolymers and formation of PIC micelles with PLL. Reprinted with permission from ref (61).

Figure $14 \mathrm{CuAAC}$ stabilization of alkyne-modified micelles with azido-dendrimer for the preparation of shell cross-linked NP. Reprinted with permission from ref (65). 
Figure 15 Preparation of immuno-NP via SPAAC decoration. [PEGO (poly(ethylene glycol)octyne); EDC (1-ethyl-3-(3-dimethylaminopropyl)carbodiimide)].

Figure 16 Preparation of PS- $b$-PAA polymersomes with peripheral azide groups and their functionalization with Alk-EGFP. [TBAF (tetra- $n$-butylammonium fluoride)]. Reprinted with permission from ref (77).

Figure 17 General approach for the surface functionalization of vesicles with dendritic wedges. Reprinted with permission from ref (78).

Figure $18 \mathrm{CuAAC}$ strategy for the preparation of DOX-loaded, folate-conjugated PCN [DXR = doxorrubicin (DOX)]. Reprinted with permission from ref (81).

Figure 19 Schematic representation of the $\mathrm{LbL} / \mathrm{CuAAC}$ preparation of triazole cross-linked $\mathrm{pH}-$ responsive polymer microcapsules from a silica template. Reprinted with permission from ref $(83)$.

Figure 20 Preparation of (PVP/PMA-thiol/PVP/PMA-Alk)-coated microparticles by LbL assembly, followed by PEGylation, cross-linking stabilization via TEC, and removal of the PVP layers and silica template. Reprinted with permission from ref (87).

Figure 21 Synthesis of (allyloxy)12cucurbit[6]uril polymer nanocapsules via TEC cross-linking. Reprinted with permission from ref (90). 
Figure 22 Functionalization of AuNP with a lipase via CuAAC. Reprinted with permission from $\operatorname{ref}(98)$.

Figure 23 Schematic representation of the preparation of theranostic and multimodal iron oxide NP. [NIR (near-infrared); paclitaxel = taxol. DiI (dialkylcarbocyanine fluorophores)]. Reprinted with permission from ref (117).

Figure 24 SPAAC surface functionalization of QD in the presence and absence of $\mathrm{Cu}(\mathrm{I})$, and effect of reaction conditions on QD luminescence. Reprinted with permission from ref (125).

Figure 25 Labeling of cells with preformed QD-EGF constructs a). In situ conjugation of QD to EGF on live cells via tetrazine-norbornene Diels-Alder cycloaddition b). Reprinted with permission from ref (126).

Figure 26 Schematic representation of the preparation of Janus-type SiNP. Reprinted with permission from ref (130).

Figure 27 Schematic representation of the SPAAC conjugation of a cyclooctyne/alkyne peptide to azido-functionalized fluorescent SiNP, and their further CuAAC labeling with an azido-FRET acceptor. [PEP (peptide)]. Reprinted with permission from ref (133).

Figure 28 Synthesis of CD-modified SWCNT. Reprinted with permission from ref (139).

\section{Click Chemistry for Drug Delivery Nanosystems}


Figure $29 \mathrm{CuAAC}$ functionalized fullerenes prepared by the groups of Isobe and Nakamura (left), and Nierengarten (right). Reprinted with permission from ref (145) and (147).

Figure 30 Functionalization of fullerene hexaadducts by means of sequential CuAAC and TEC. Reprinted with permission from ref (152).

Figure 31 Functionalization of CPMV with biologically relevant ligands, polymers, and imaging agents via CuAAC.

Figure 32 Rationale and strategy for fabricating a hybrid protein cage/dendritic structure. Reprinted with permission from ref (162). 


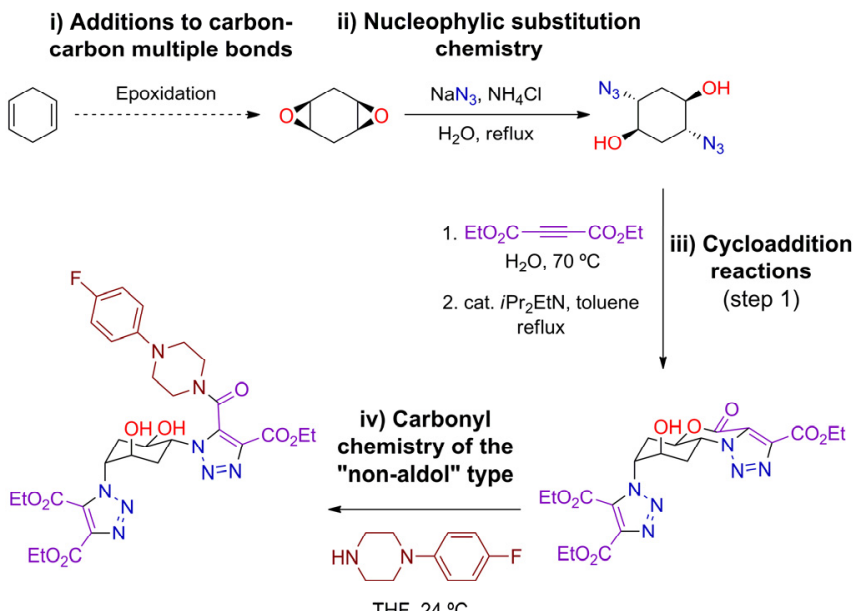

$1 \quad$ Figure 1

THF, $24{ }^{\circ} \mathrm{C}$

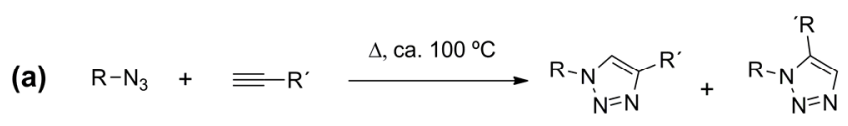

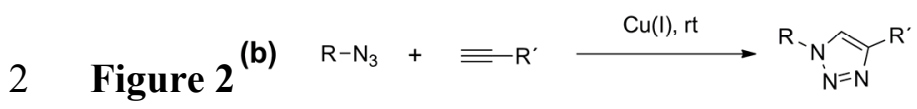

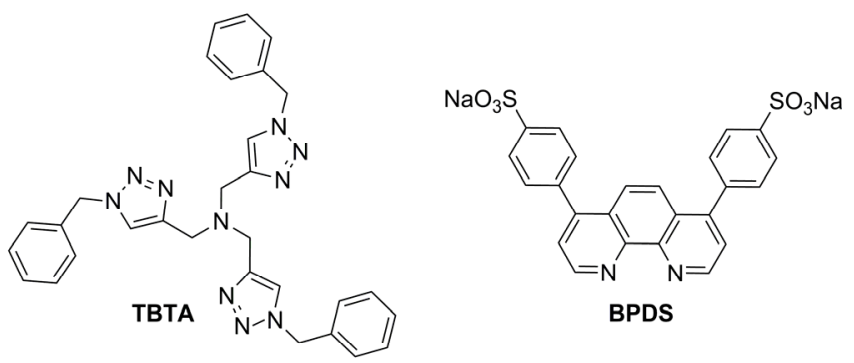

$3 \quad$ Figure 3

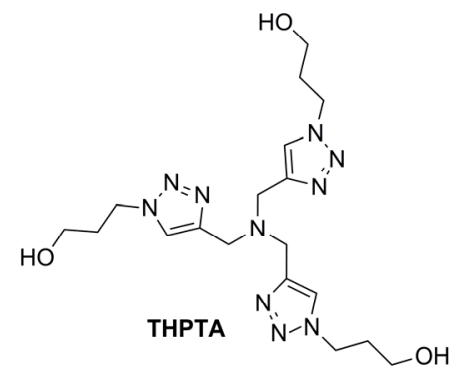

Click Chemistry for Drug Delivery Nanosystems 


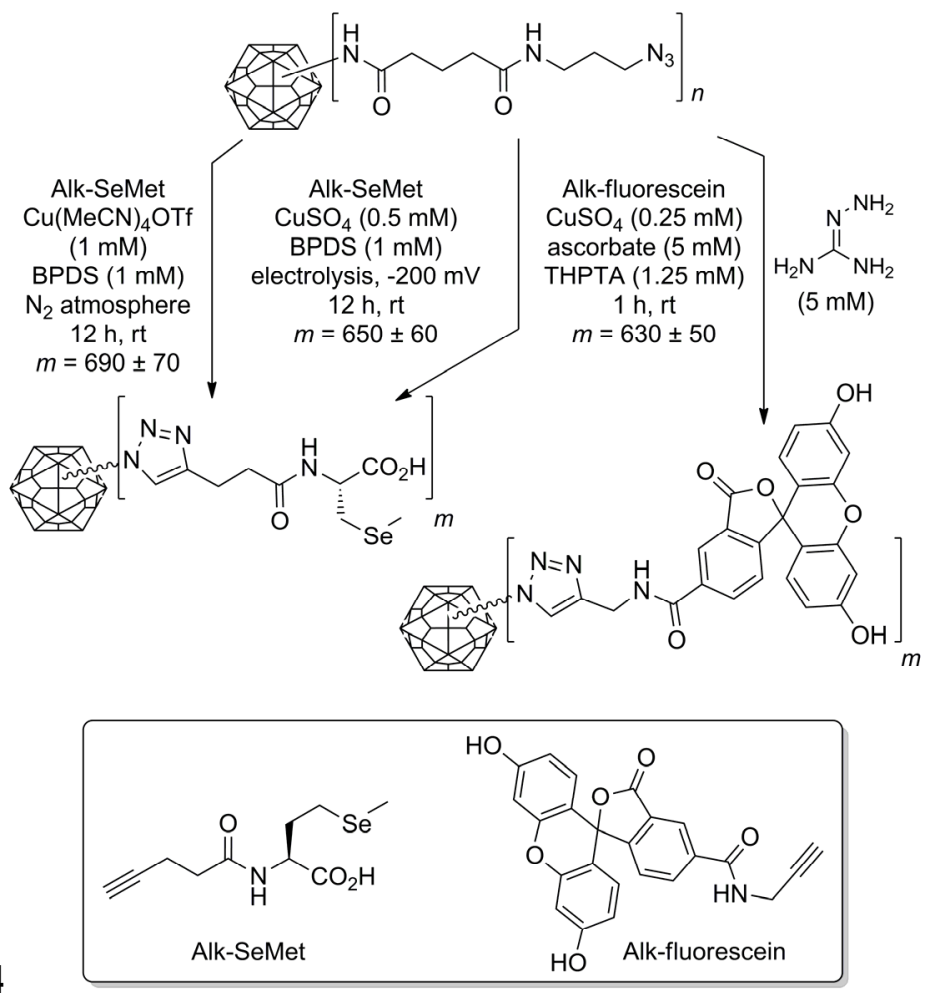

(a)

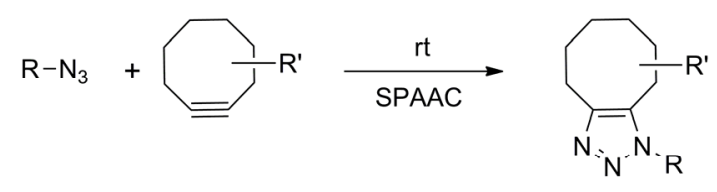

(b)<smiles>[R]C1Cc2ccccc2C#Cc2ccccc21</smiles><smiles>[R]C(=O)N1Cc2ccccc2C=Cc2ccccc21</smiles>

2 Figure 5<smiles>[R]N1C(=O)c2ccccc2C#Cc2ccccc21</smiles>

BARAC<smiles>[R]OC[C@@H]1[CH]CCC#CCC1</smiles>

$\mathrm{BCN}$<smiles>[R20]c1ccc2c(c1)CCc1cc([R])ccc1C1C(=O)C21</smiles> 
Lallana Page 80

$1 \quad$ Figure 6
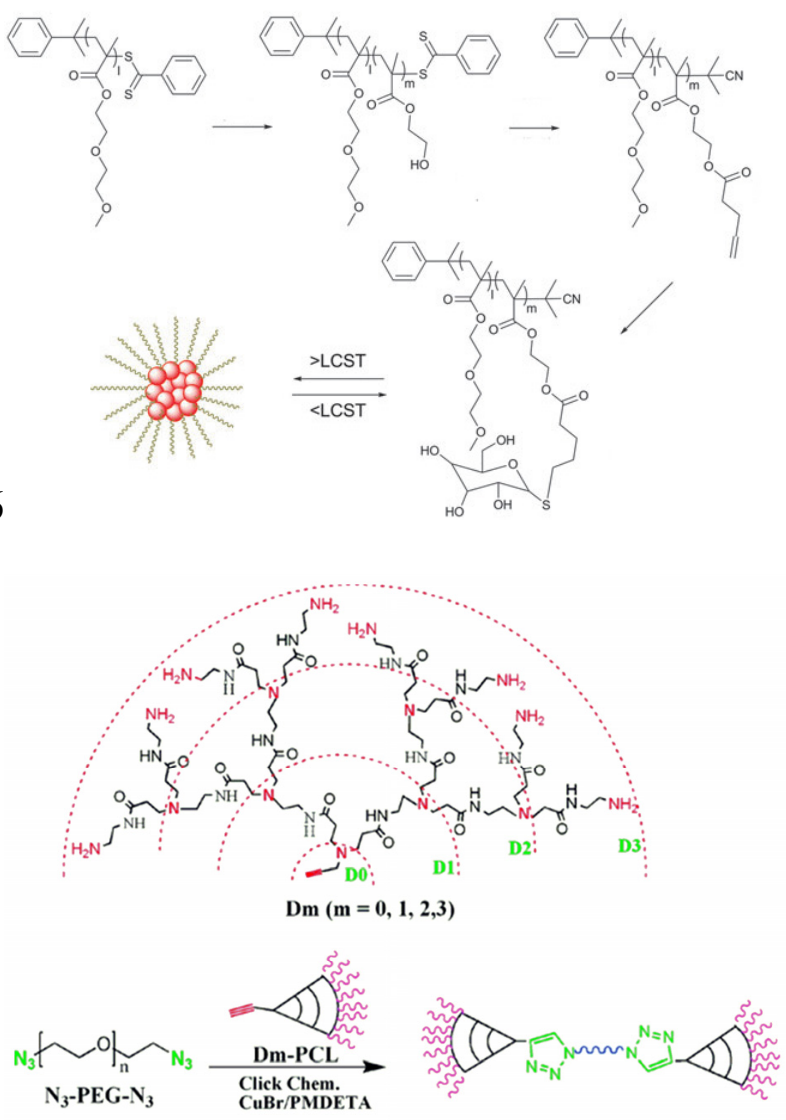

$2 \quad$ Figure 7

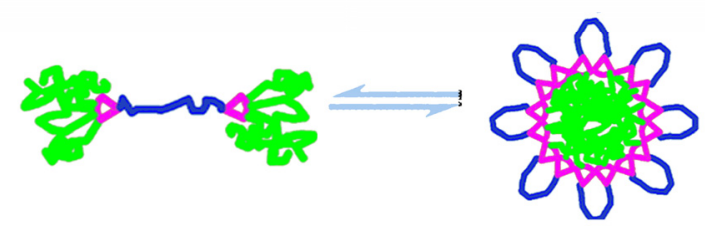


Lallana Page 81

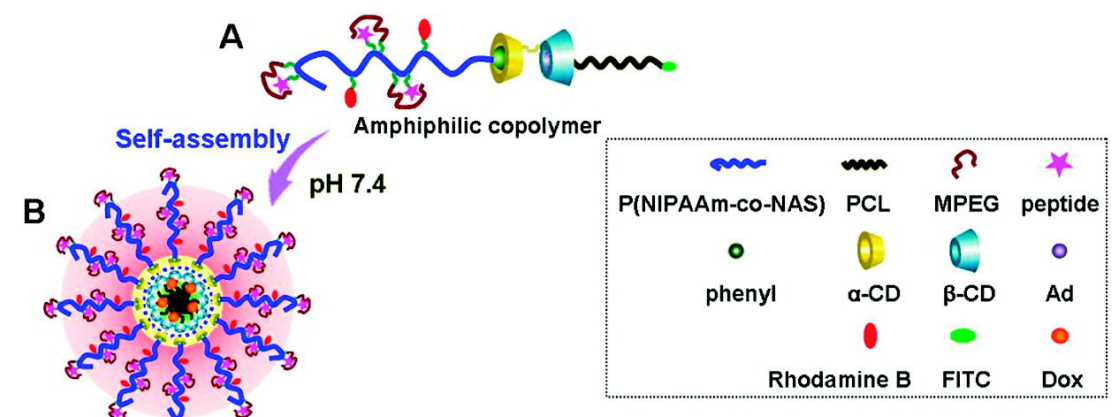

NCCM with protected ligands

"Tumor-

Triggering"

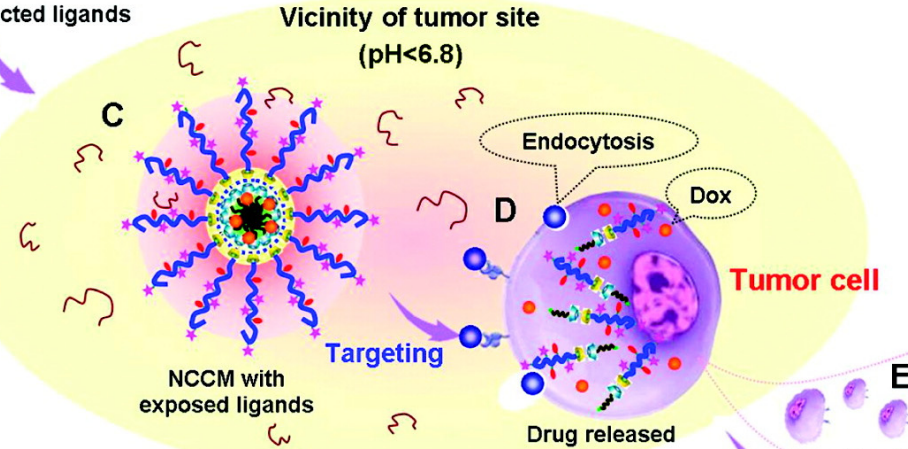

Figure 8

Apoptosis Dead tumor cells

2 Figure 9

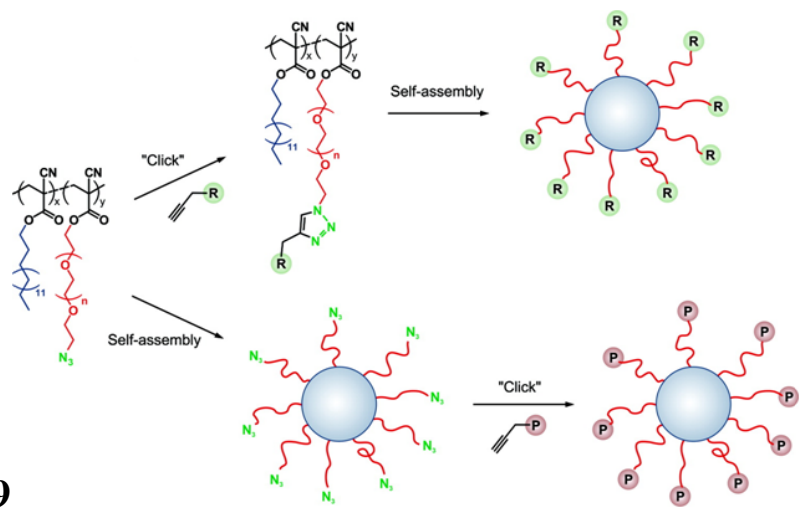

Click Chemistry for Drug Delivery Nanosystems 
Lallana Page 82

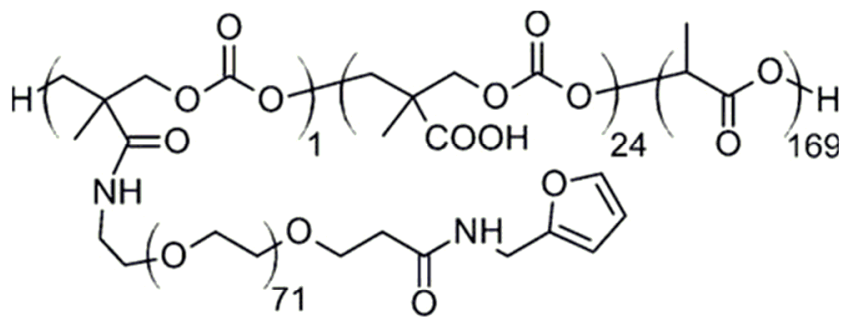

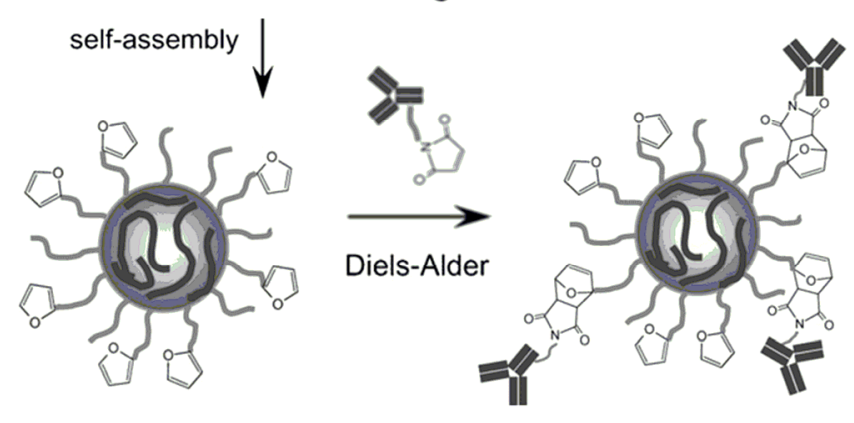

Figure 10

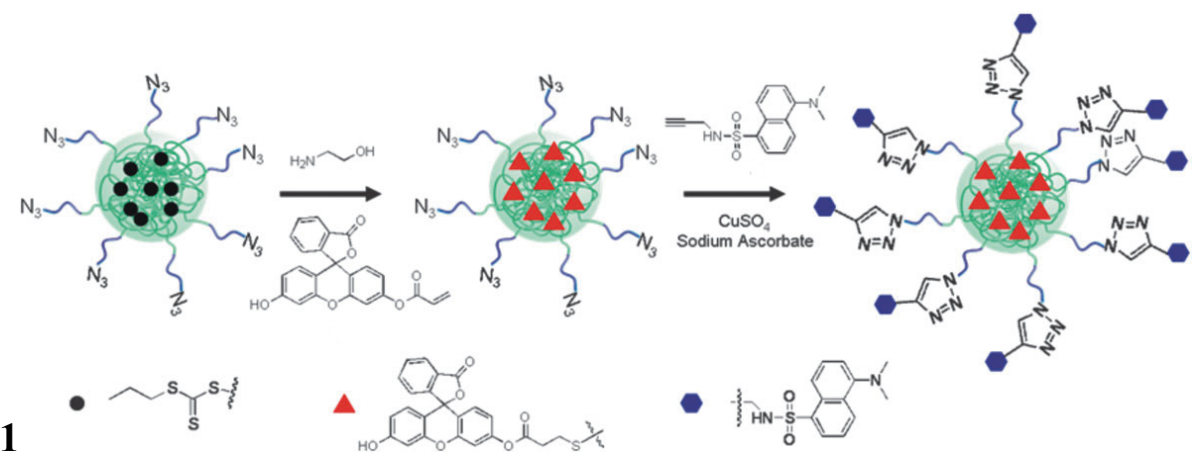

2 Figure 11

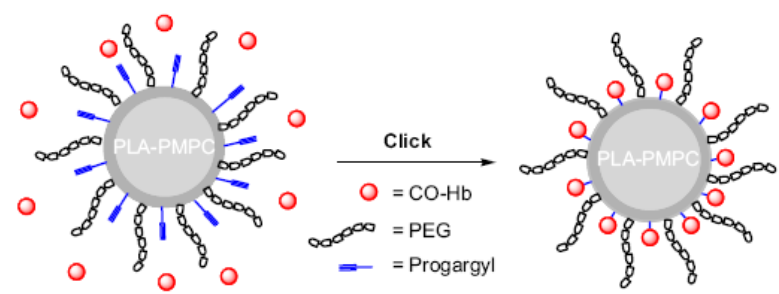

$3 \quad$ Figure 12

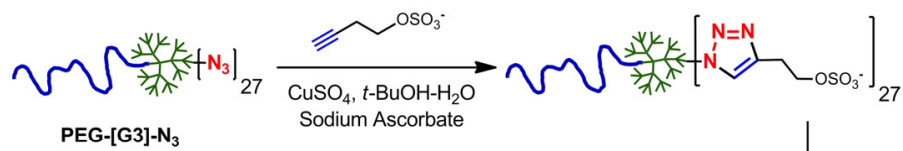

$4 \quad$ Figure 13

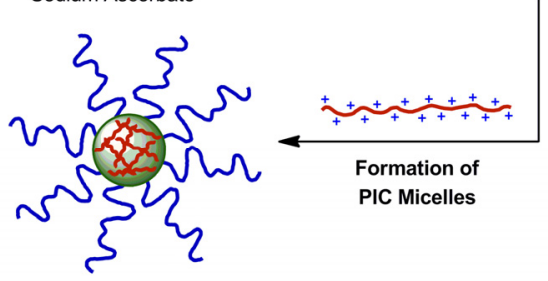

Click Chemistry for Drug Delivery Nanosystems 
Lallana Page 83

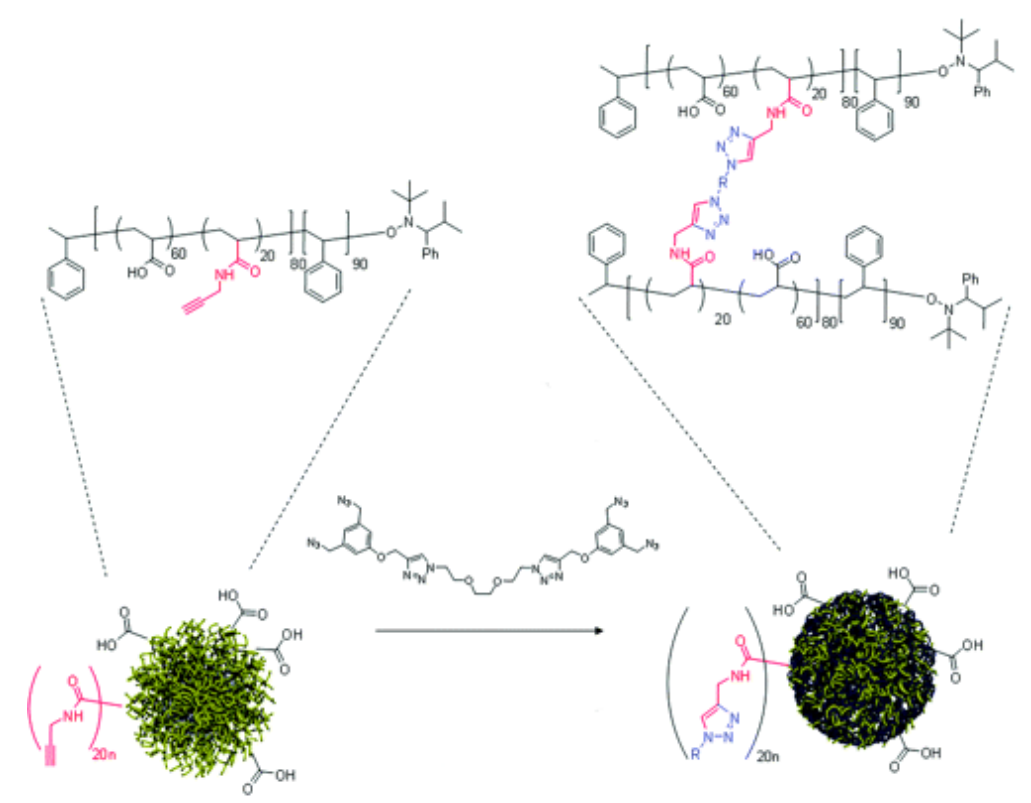

$1 \quad$ Figure 14

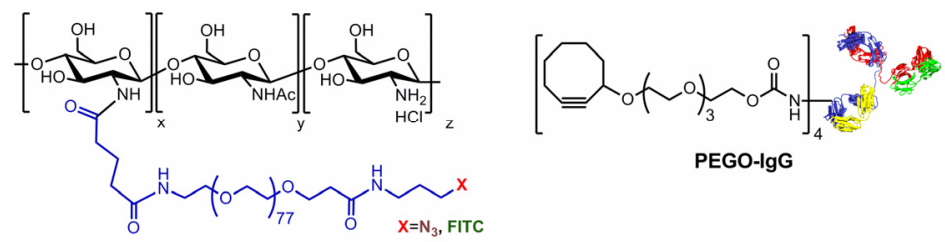

2 Figure 15

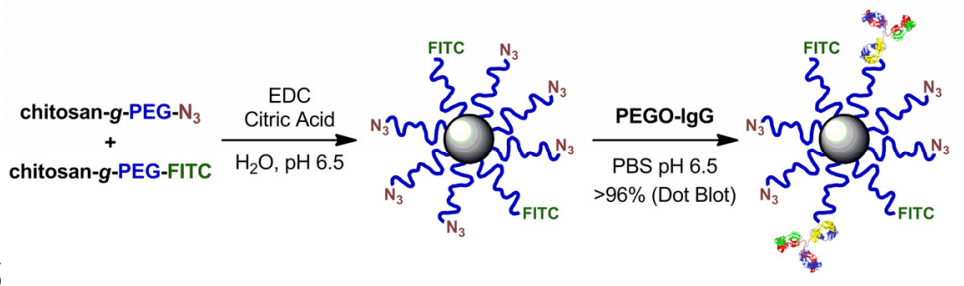

Click Chemistry for Drug Delivery Nanosystems 

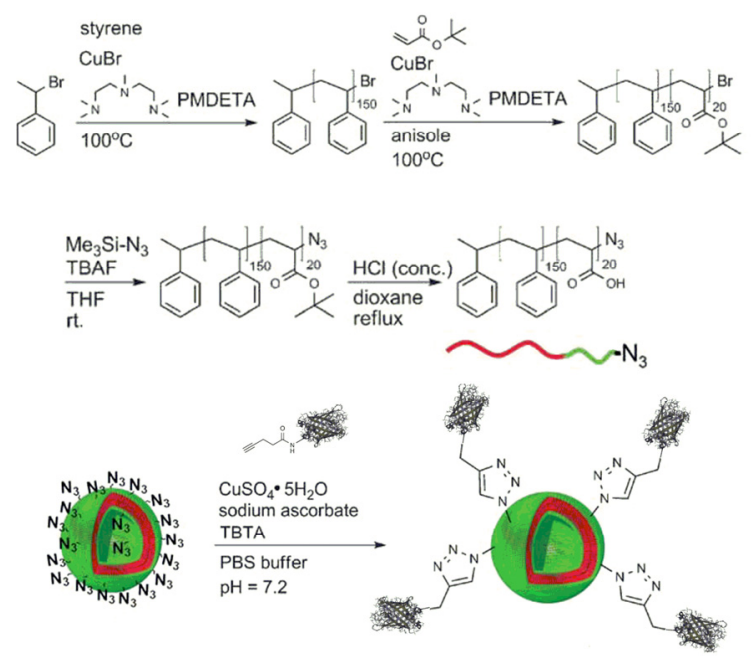

1 Figure 16
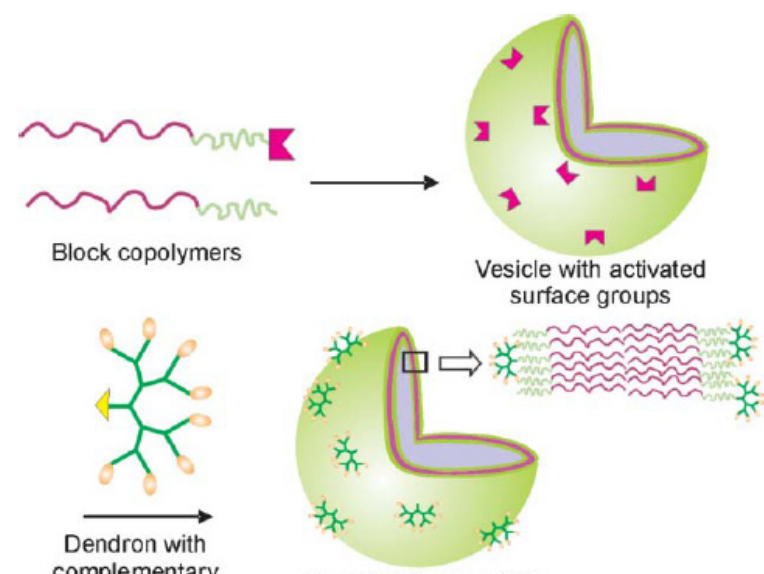

$2 \quad$ Figure 17

complementary

Functionalized vesicle 
Lallana Page 85
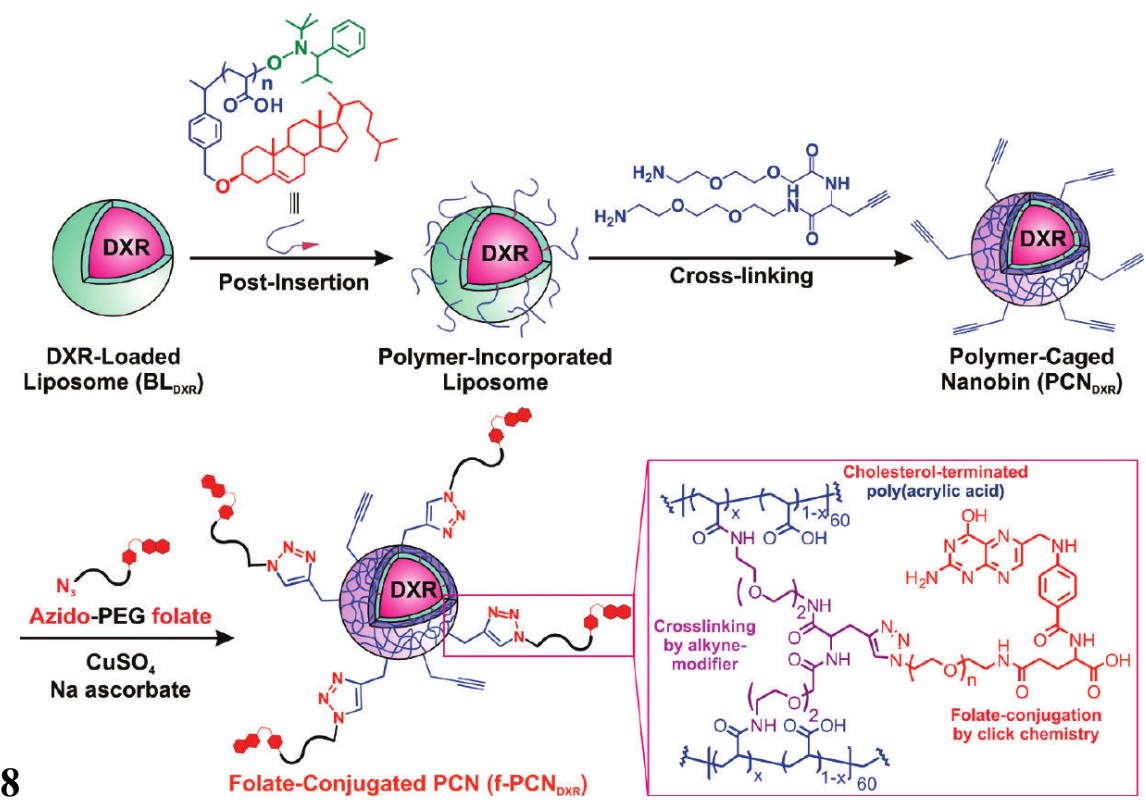

Figure 18

Folate-Conjugated PCN (f-PCN $\mathrm{N}_{\mathrm{DXR}}$ )

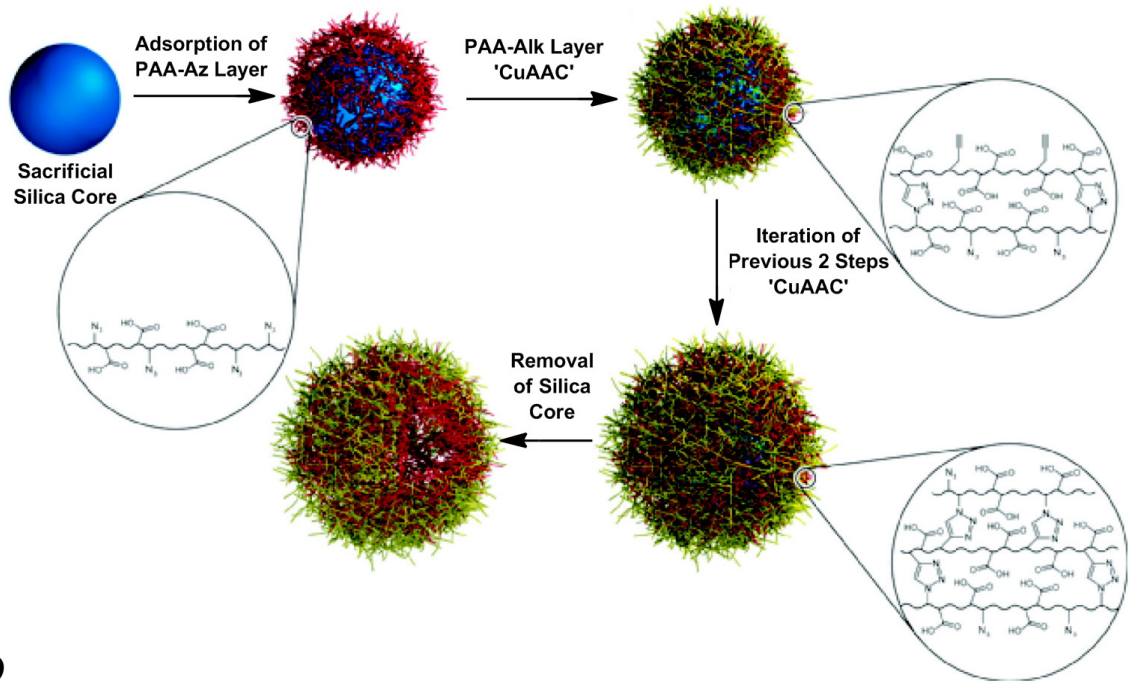

2 Figure 19

Click Chemistry for Drug Delivery Nanosystems 


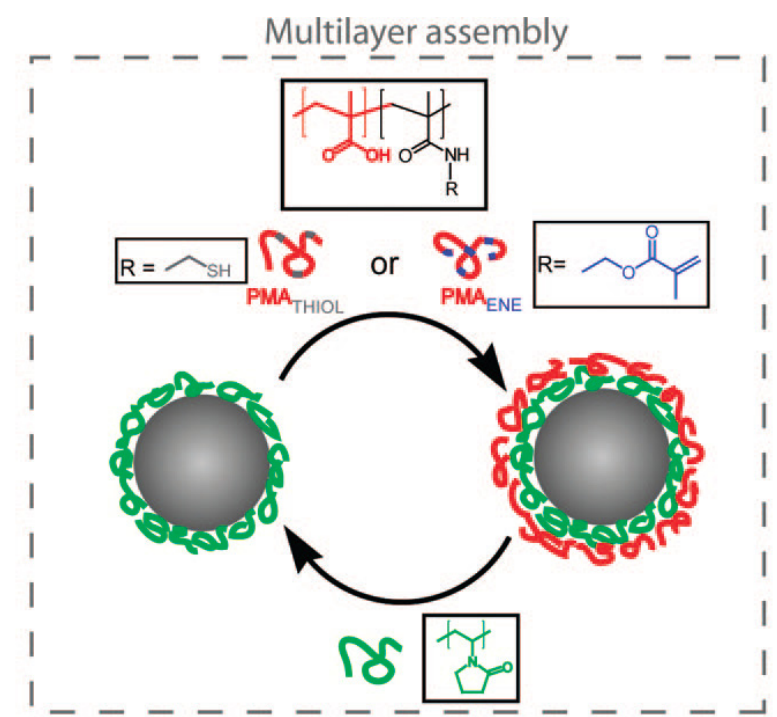

Thiol-ene based stabilization and PEGylation

Figure 20|
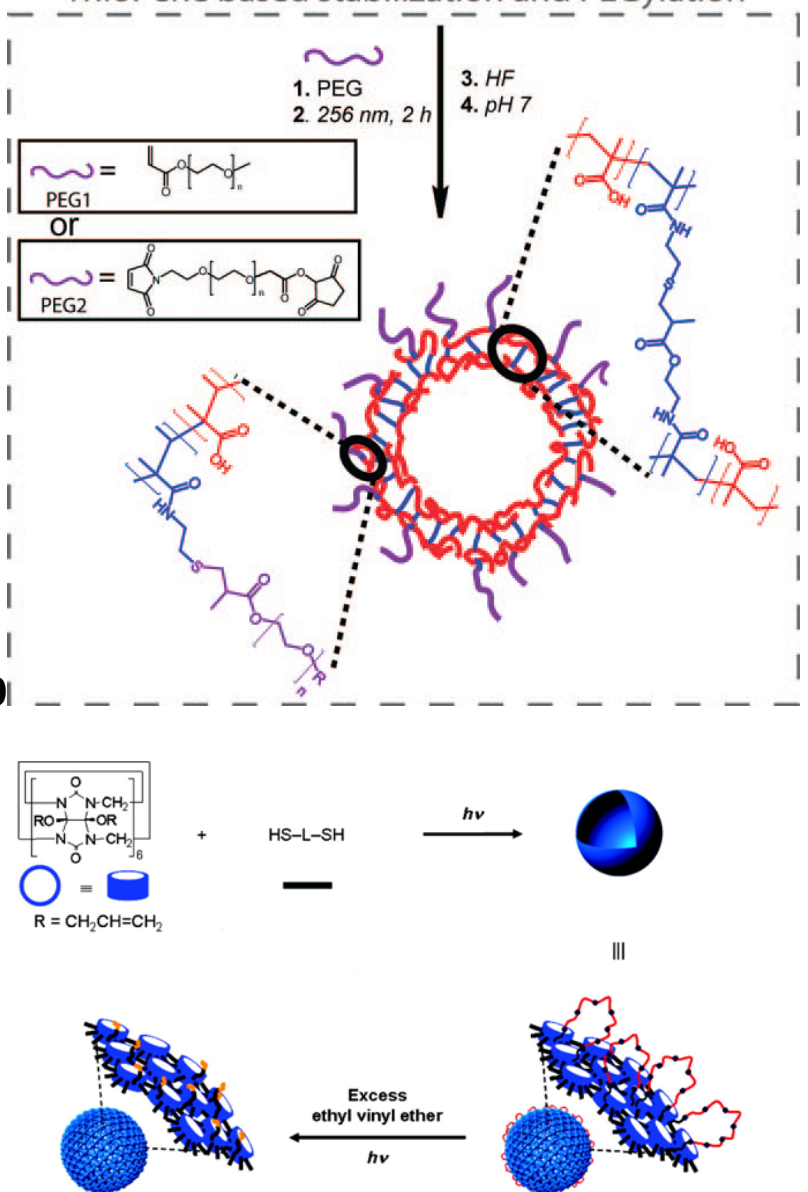

Figure 21

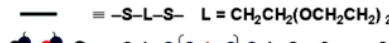

Click Chemistry for Drug Delivery Nanosystems 

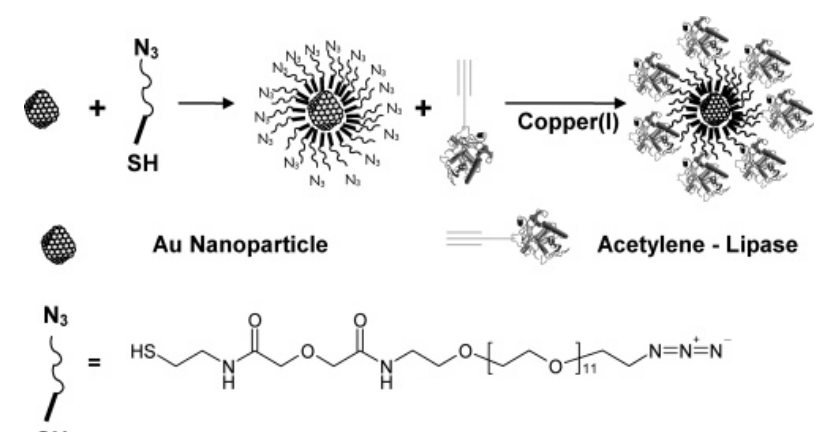

1 Figure 22

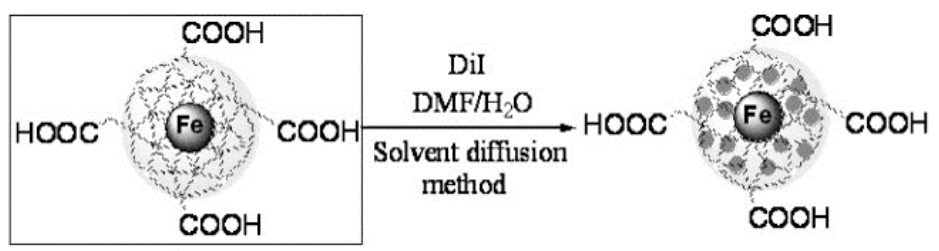

Carbodiimide Propargyl amine chemistry EDC/NHS

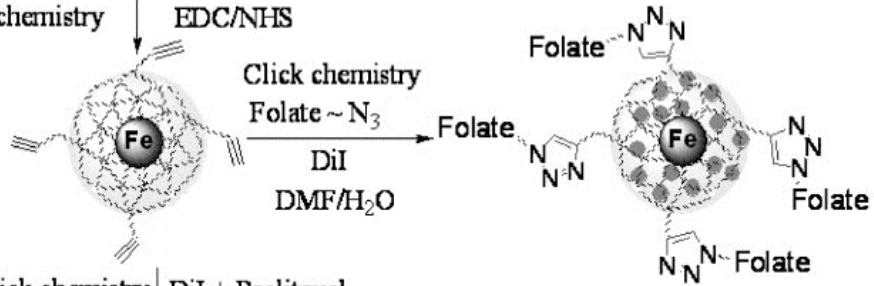

Click chemistry $\mid$ DiI + Paclitaxel

$\mathrm{N}_{\mathrm{N}} \mathrm{N}$. Folate

Folate $\sim \mathrm{N}_{3} \quad \mathrm{DMF} / \mathrm{H}_{2} \mathrm{O}$

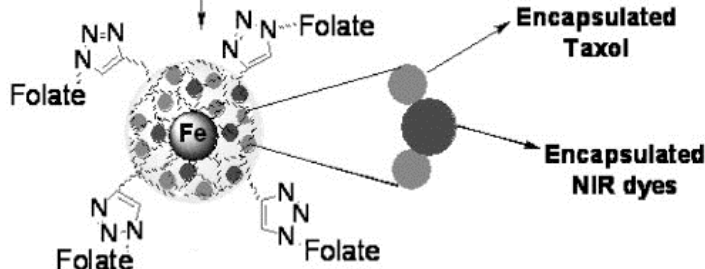

Figure 23 Folate

Click Chemistry for Drug Delivery Nanosystems 
Lallana Page 88

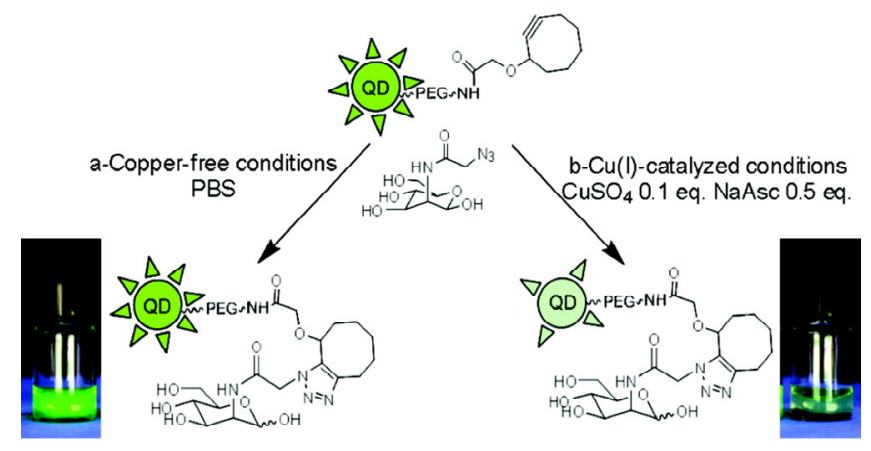

\section{$1 \quad$ Figure 24}

(a)

2 Figure 25

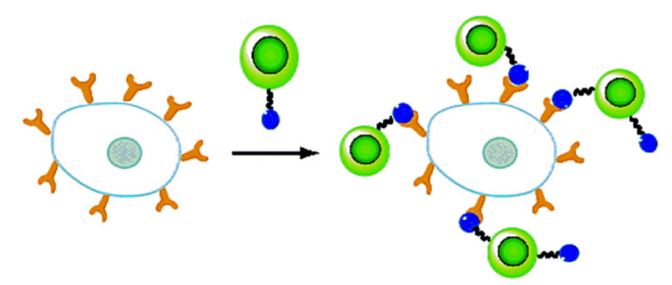

(b)
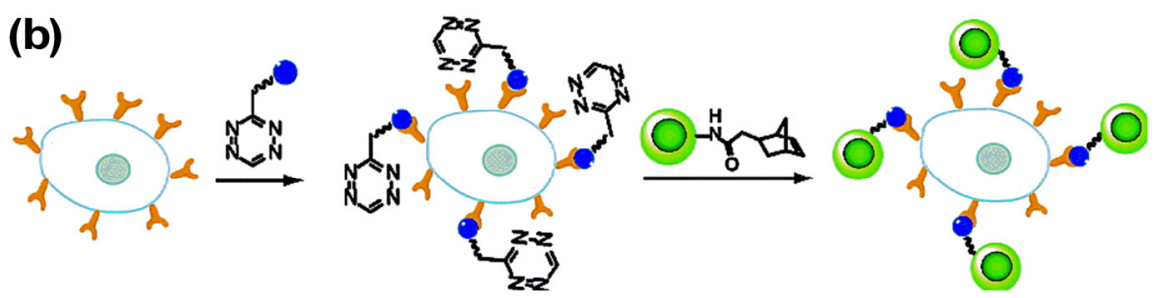
Lallana Page 89

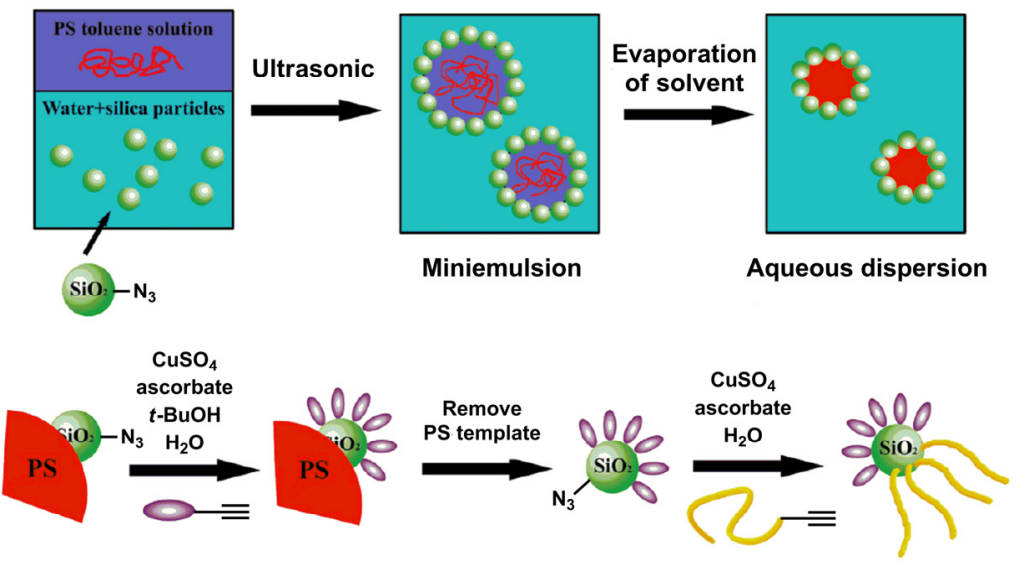

Figure 26
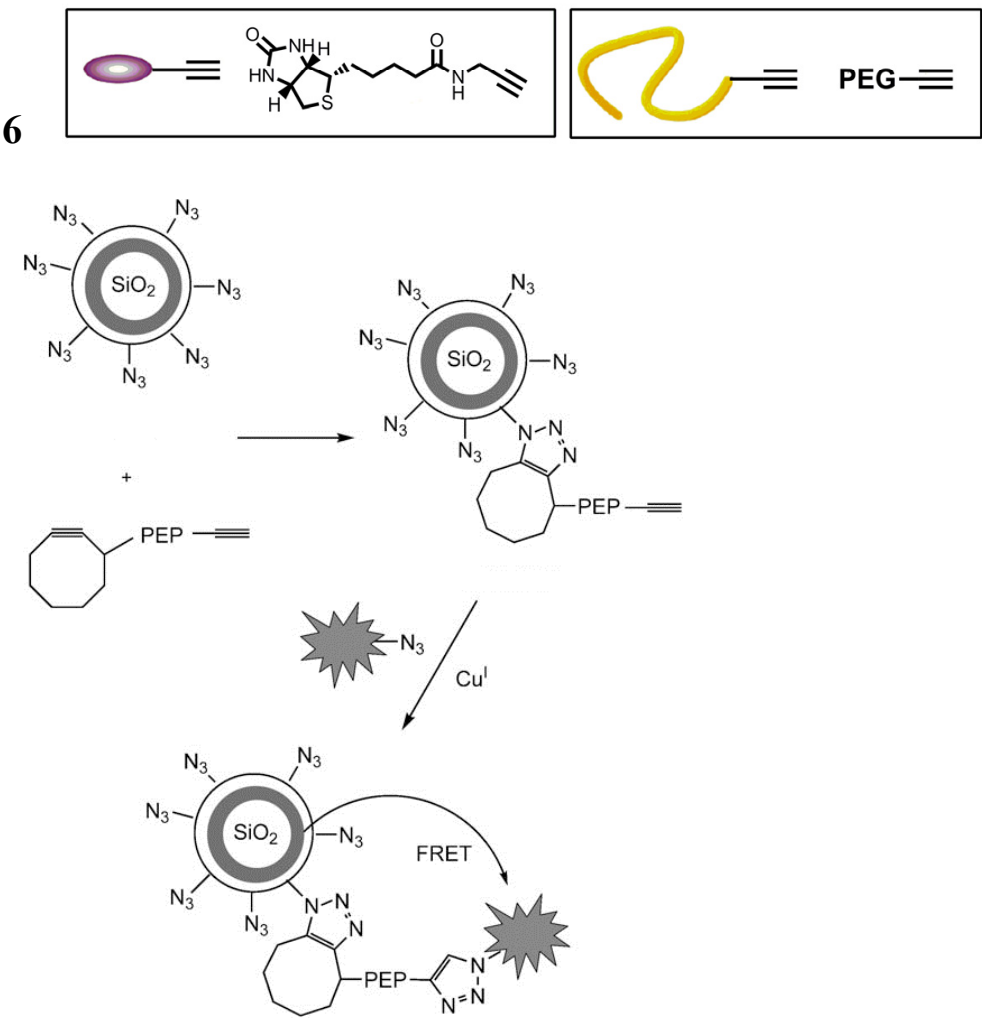

$2 \quad$ Figure 27

Click Chemistry for Drug Delivery Nanosystems 
Lallana Page 90

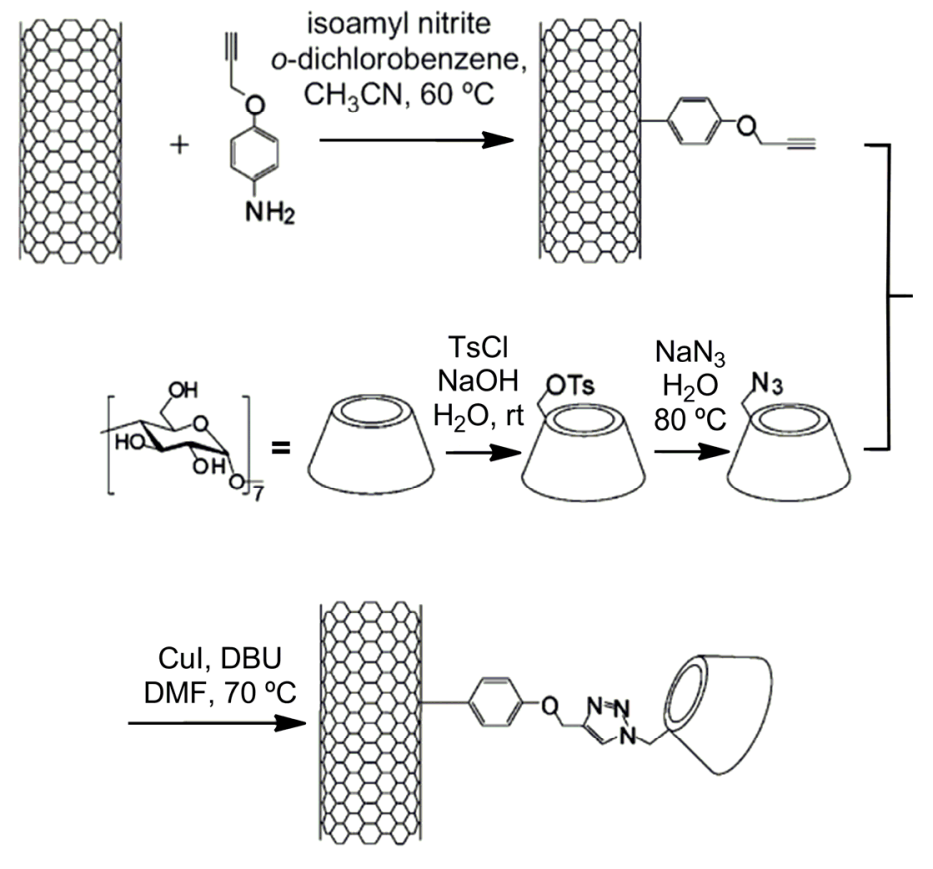

Figure 28

2 Figure 29
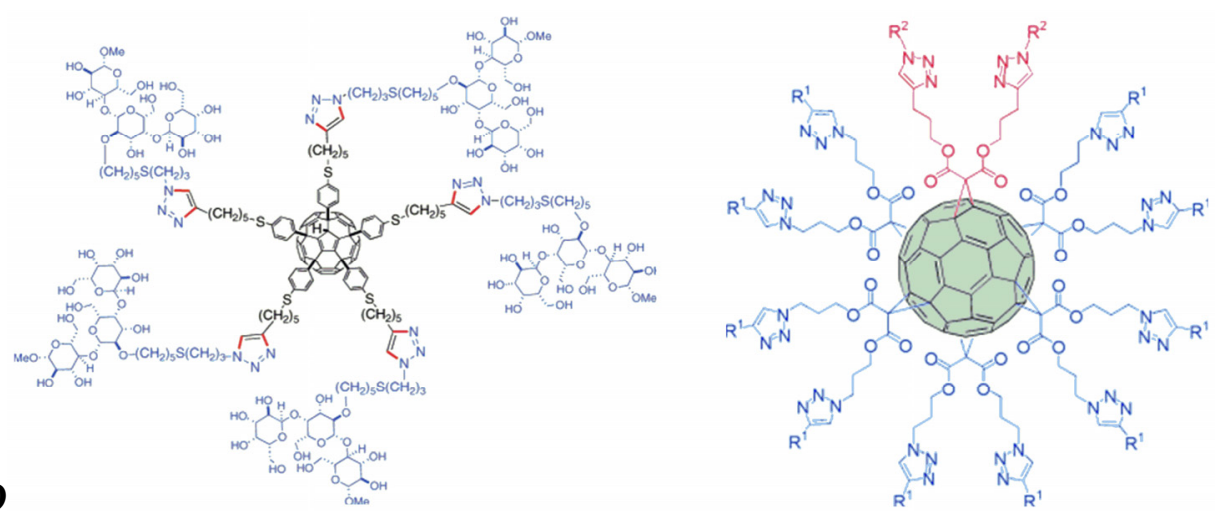

Figure 30

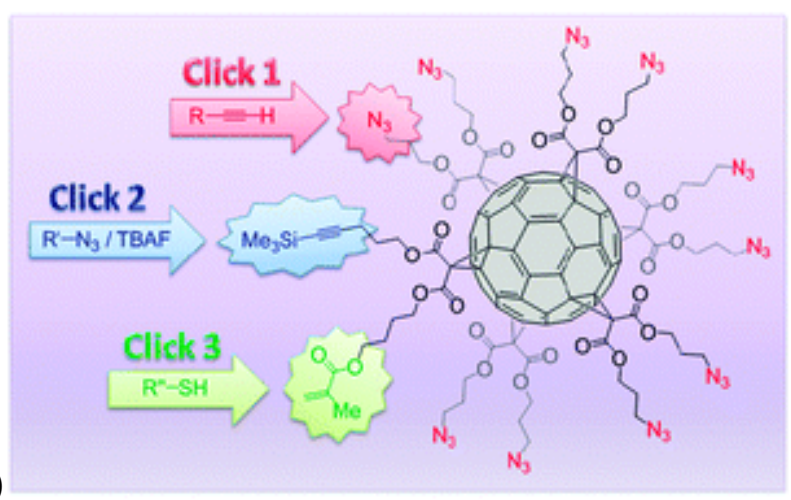

Click Chemistry for Drug Delivery Nanosystems 

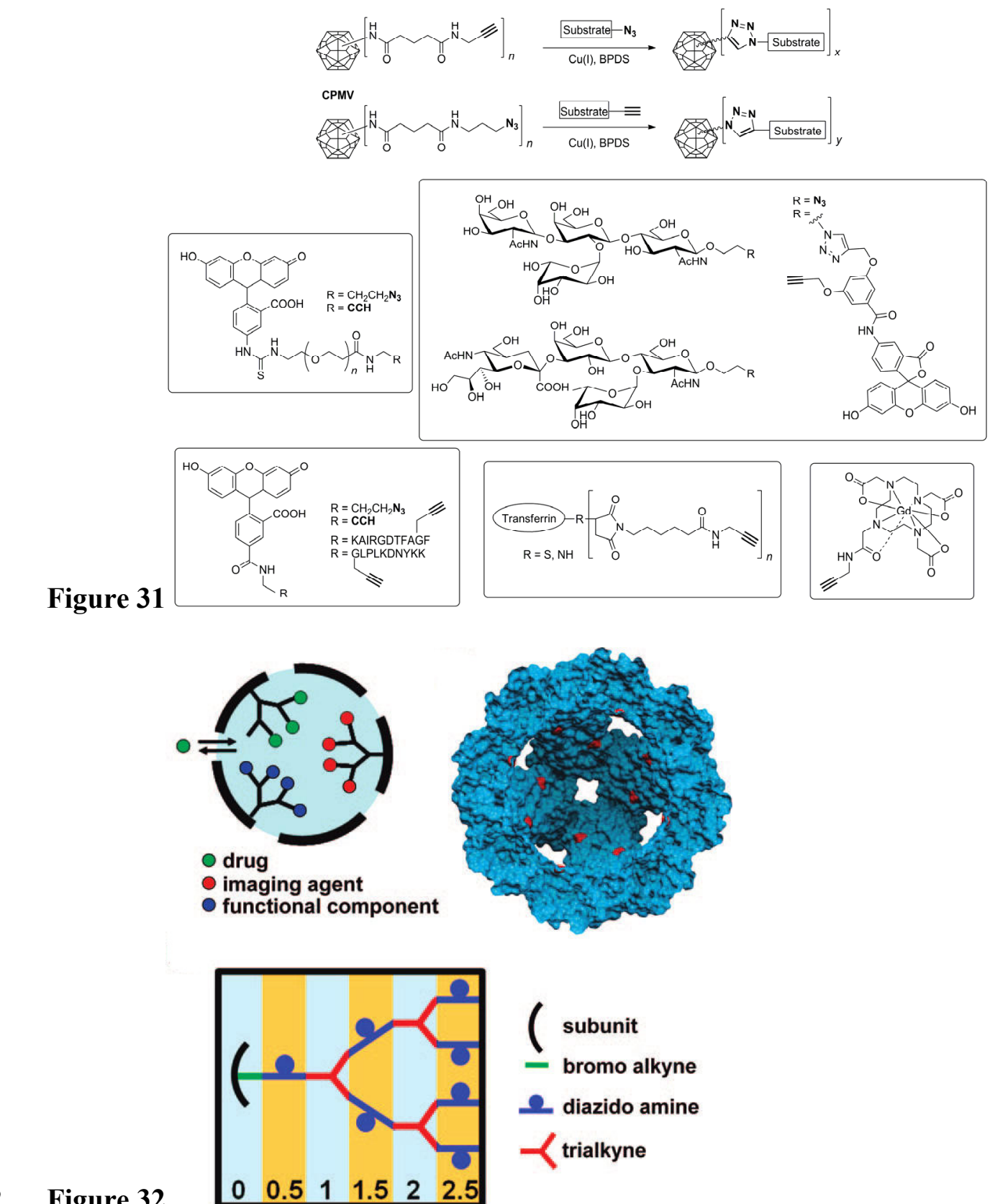